\title{
Family Descriptions of the Influence of Cultural Factors on Transitions in Serious Illness
}

Veronica Gallo

Follow this and additional works at: https://researchrepository.wvu.edu/etd

\section{Recommended Citation}

Gallo, Veronica, "Family Descriptions of the Influence of Cultural Factors on Transitions in Serious IIIness" (2015). Graduate Theses, Dissertations, and Problem Reports. 5643.

https://researchrepository.wvu.edu/etd/5643

This Dissertation is protected by copyright and/or related rights. It has been brought to you by the The Research Repository @ WVU with permission from the rights-holder(s). You are free to use this Dissertation in any way that is permitted by the copyright and related rights legislation that applies to your use. For other uses you must obtain permission from the rights-holder(s) directly, unless additional rights are indicated by a Creative Commons license in the record and/ or on the work itself. This Dissertation has been accepted for inclusion in WVU Graduate Theses, Dissertations, and Problem Reports collection by an authorized administrator of The Research Repository @ WVU.

For more information, please contact researchrepository@mail.wvu.edu. 
Family Descriptions of the Influence of Cultural Factors on Transitions in Serious Illness

\author{
Veronica Gallo
}

Dissertation submitted
to the School of Nursing
at West Virginia University

in partial fulfillment of the requirements for the
degree of Doctor of Philosophy in Nursing

Committee Members

Joy Buck, Ph.D, Chair

Gina Maiocco, Ph.D. Elisabeth Shelton, Ph.D.

Mary Jane Smith, Ph.D.

Debra Wiegand, Ph.D.

Department of Nursing

\title{
Morgantown, West Virginia 2015
}

Keywords: Family, transitions, serious illness, culture Copyright 2015 Veronica Gallo 


\begin{abstract}
Family Descriptions of the Influence of Cultural Factors on Transitions in Serious Illness Veronica Gallo

Nearly 65 million Americans served as care provider to an ill or disabled adult, and over 1.6 million Americans participated in the care of a family member in hospice. Families experience transitions, or changes, throughout the serious illness trajectory. Throughout transitions in serious illness, families participate in the care, support, and decision-making for their ill family member. Families' lives are often disrupted during this time and families experience emotional, physical and psychological distress during transitions. In spite of this, families are rarely included in care, and little is known about how families make sense of transitions. Culture, and the factors that shape it, can impact family interpretations of illness and meanings of illness transitions. This qualitative study explored family descriptions of the influence of four cultural factors (kinship/family, knowledge systems, community/social systems, and faith/religion) on transitions in serious illness during an understudied transitional time span, from the point of diagnosis up to the EOL phase. Eleven participant interviews were gathered from twelve family members of persons recently deceased due to serious illness. Data collection and analysis were guided by the concepts of culture and transitions in illness and conventional content analysis methods. Seven themes emerged from the data that described the influence of cultural factors on family transitions in serious illness: (1) family takes care of family; (2) we're "going to fight this;" (3) "seeing" and experience were believing; (4) families "figured it out;" (5) community eased the family burden; (6) health professionals both eased and hindered family transitions; and (7) "God was always in the background." Findings support the need for provision of patientcentered, family-focused, culturally congruent nursing care to seriously ill persons and their families across the illness continuum. Implications for nursing education and research are also discussed.
\end{abstract}




\section{Dedication}

This dissertation is dedicated to my husband Sam and our four daughters, Caroline, Jenna, Anna, and Mia. Without their understanding, support, and patience, this manuscript would not be possible.

\section{Acknowledgements}

Without the support of several important people, this research could not have been completed. First, I would like to acknowledge the members of my dissertation committee. Dr. Gina Maiocco, Dr. Elisabeth Shelton, Dr. Mary Jane Smith, and Dr. Debra Wiegand; thank you for your continual support and guidance throughout this venture. To Dr. Joy Buck, my committee chair, I would like to express a special thank you. Your constant encouragement and praise served as an inspiration for me to continue on and complete this sometimes arduous endeavor.

I would like to acknowledge my two classmates, Dr. Courtney Pate and Dr. Shavon Dardon for their continual support. I would also like to thank my friends and co-workers for listening and offering support in so many ways. A special thank you to co-workers and friends who helped recruit participants. Also, a special thank you to the families who agreed to participate in this study. Without their help, this work could not have been completed.

I would also like to thank my family for their support. I would like to thank my parents for their continual encouragement and interest in my research. To my mother, Linda, and sisters, Jennifer and Nikki, thank you for understanding when I couldn't talk or visit because I had to “work on my dissertation." To my sister, Jennifer, a special thank you for lending an ear when I needed to "vent" and for lifting me up and pushing me forward when I hit one of many roadblocks. Thank you to my father-in-law, John, for your continual support and praise, and for 
helping care for my children throughout this process. To my children, first an apology for having to pass on several of your activities, and for not being able to be the "go-to" mom because I had to "work on my dissertation." Thank you, girls, for understanding and loving me anyway. A special thank you to my husband, Sam. You have been a huge support for the past several years. Thank you for understanding and encouraging me along the way. Finally, I would like to remember my mother-in-law Peggy. Our family's experiences with her illness served as an inspiration for this study. We miss her every day. 


\section{Table of Contents}

CHAPTER 1-INTRODUCTION..............................................................1

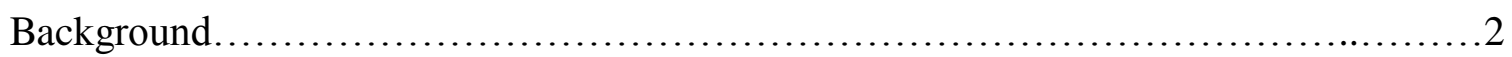

Significance...........................................................6

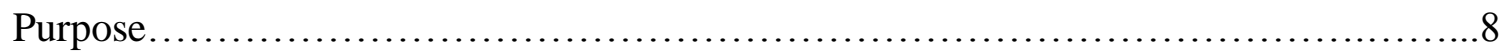

Research Question......................................................

Definitions.................................................... 9

Method....................................................................11

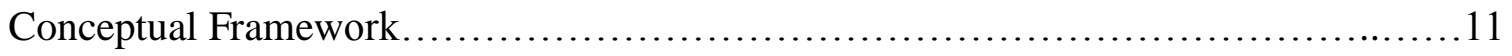

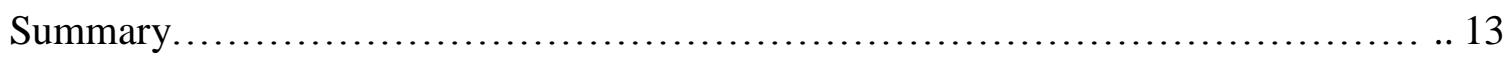

CHAPTER 2-REVIEW OF THE LITERATURE......................................15

Literature Search Process.................................................15

Literature Findings.....................................................

Conceptual and Theoretical............................................ 16

Methodological........................................................ 19

Quantitative/Mixed Methods....................................19

Qualitative.................................................20

Empirical............................................................ 23

Family transitions in advanced stages of illness ........................24

Family needs and values when entering EOL phase ......................28

Divergent perspectives of transitions in advanced serious illness .........29

Culture and family transitions at EOL .................................30

Synthesis................................................................. 33

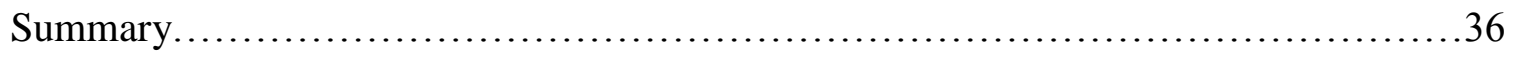




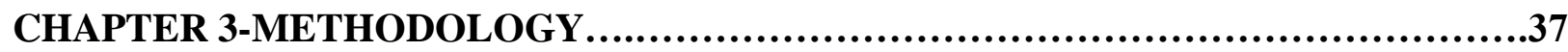

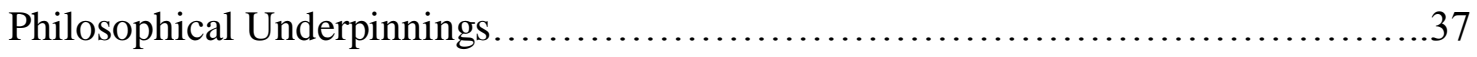

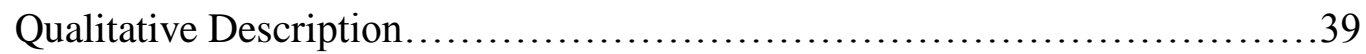

Content Analysis..........................................................

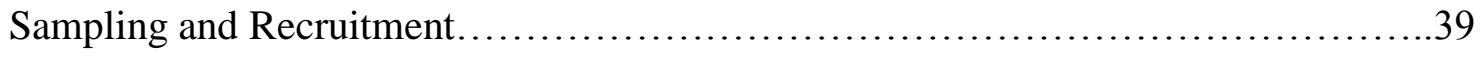

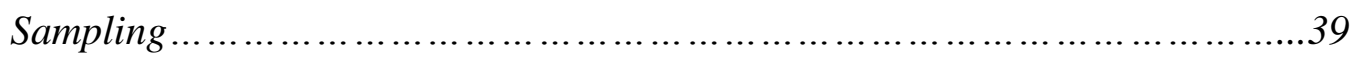

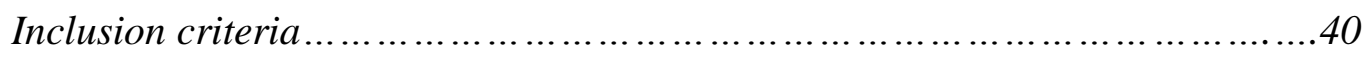

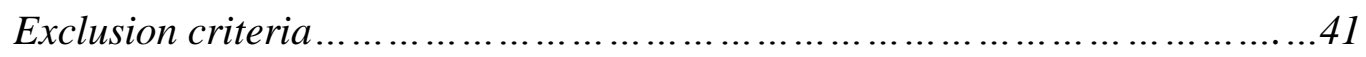

Recruitment procedures...............................................41

Data Collection............................................................... 43

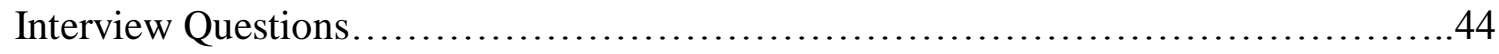

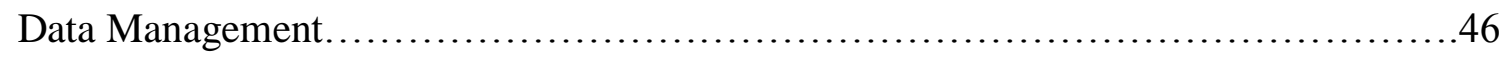

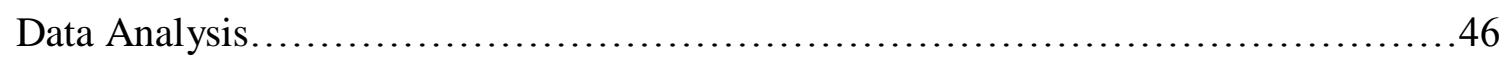

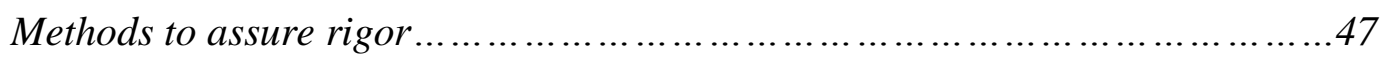

Human Rights Considerations..................................................49

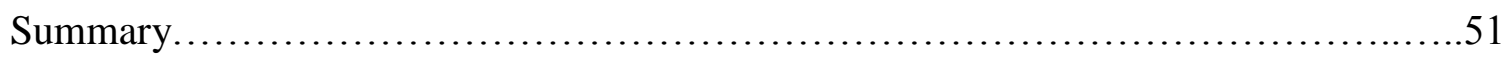

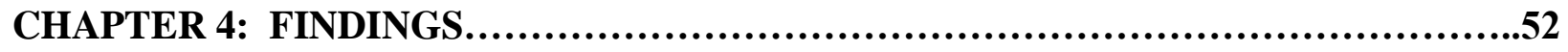

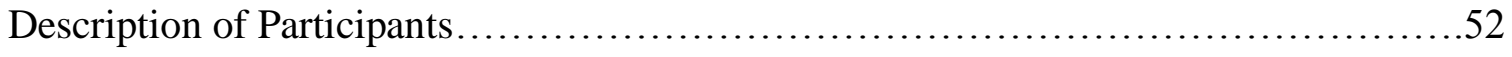

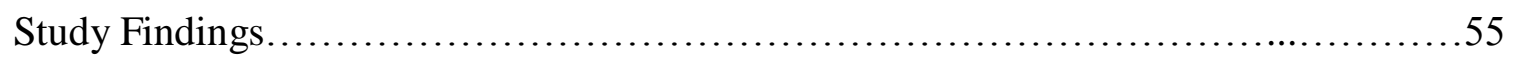

The influence of family/kinship on illness transitions................................55

Theme 1. Family takes care of family...................................58

Theme 2. We're "going to fight this" ..................................65

The influence of knowledge systems on illness transitions...........................67

Theme 3. "Seeing" and experience were believing.....................67

Theme 4. Families "figured it out"....................................73 
DESCRIPTIONS OF THE INFLUENCE OF CULTURAL FACTORS ON TRANSITIONS IN SERIOUS ILLNESS

The influence of community/social systems on illness transitions...................76

Theme 5. Community eased the family burden..........................77

Theme 6. Health professionals both eased and hindered family transitions..78

The influence of faith/religion on illness transitions $\ldots \ldots \ldots \ldots \ldots \ldots \ldots \ldots \ldots \ldots \ldots \ldots . \ldots 5$

Theme 7. "God was always in the background".......................85

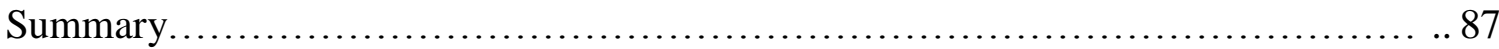

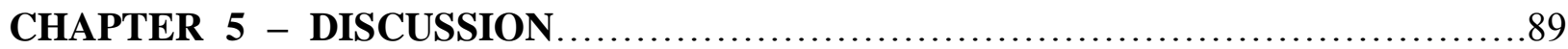

Summary of Findings................................................... 90

Findings related to Conceptual Framework.......................................99

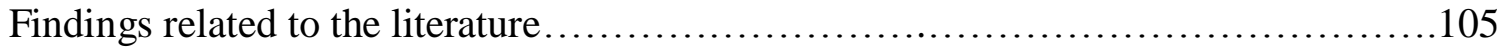

Family/Kinship.................................................. 105

Knowledge systems................................................ 108

Community/Social systems......................................... 112

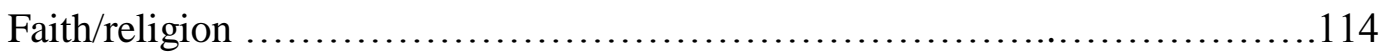

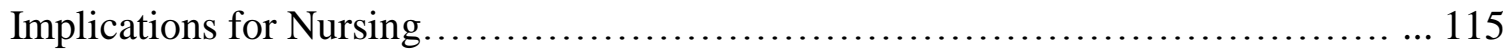

Practice.............................................................. 115

Education......................................................... 120

Research........................................................... 122

Strengths and Limitations ................................................... 124

Strengths........................................................ 124

Limitations...................................................... 126

Summary........................................................127

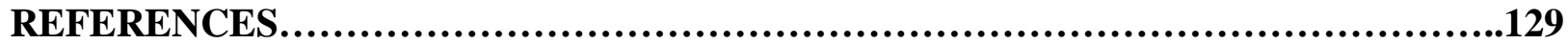

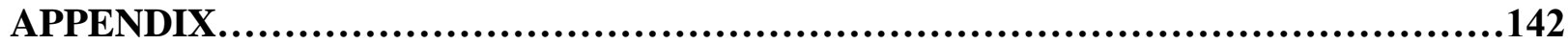

2.1 Literature review matrix .................................................. 143 
DESCRIPTIONS OF THE INFLUENCE OF CULTURAL FACTORS ON TRANSITIONS IN SERIOUS ILLNESS

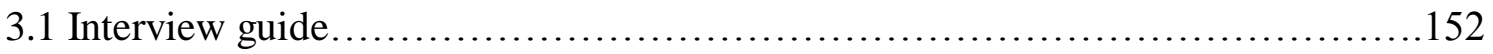

3.2 Participant letter................................................... 155

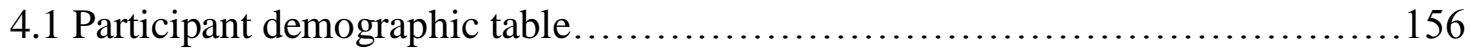




\section{Chapter 1}

Families play an integral role in the care of persons with serious illness; they serve as care providers, decision makers, and support persons to the ill person throughout the illness trajectory, and experience multiple changes or transitions along the way (Blum \& Sherman, 2010; Dosser \& Kennedy, 2012; Herbert \& Schultz, 2007; Penrod, Hupcey, Shipley, Loeb \& Baney, 2012). Families experience emotional, physical, and psychological distress throughout transitions in illness. Though family members' lives and health are often disrupted by serious illness, health professionals focus primarily on the person with serious illness; little attention is given to how families make sense out of a myriad of transitions as illness progresses to death. Deeply embedded cultural beliefs about illness, debility, and death shape an individual's interpretation and response to the decline of a family member. Such beliefs come into play when families are required to make difficult decisions on behalf of their family member, and then are left to make sense of their decisions and actions long after the ill person has died (Braun, Beyth, Ford \& McCullough., 2008; Meeker \& Jezewski, 2005; Paul \& Rattray, 2008; Phillips \& Reed, 2009). An in-depth understanding of the family's perspective of transitions in serious illness is critical to the development of interventions aimed at supporting families through them. There is little in the literature to help guide health professionals as they endeavor to provide personcentered and family-focused care. It is that gap that this research partially addressed.

Chapter one begins with an overview of the background of the problem to be studied. A description of the role that family plays in care of the seriously ill and the importance of culture to family transitions throughout serious or life-limiting illness are provided. The problem statement, prevalence and impact of the problem are given. A discussion of the method of studying the problem and the significance of the study to the discipline of nursing is included. 
The purpose of the study and research question is stated. The chapter concludes with a description of key terms and a discussion of the conceptual framework used to guide the study.

\section{Background}

In 2012, over 65 million Americans served as care providers to an ill or disabled adult (American Psychological Association [APA], 2014), and nearly 1.6 million persons participated in the care of a family member enrolled in hospice (National Hospice and Palliative Care Organization [NHPCO], 2013). Family members serve as care providers, decision makers, and support persons for their loved ones throughout the serious illness trajectory (Blum \& Sherman, 2010; Dosser \& Kennedy, 2012; Herbert \& Schultz, 2006; Penrod, et al., 2012), yet families are often the forgotten care recipients. When family does receive care, typically it is done so only after the ill person has enrolled in hospice and families are formally incorporated into the plan of care. Though families should be included in end-of-life care (EOL) of seriously ill persons, they should not be neglected in earlier phases of the illness (World Health Organization, 2007, 2012; The Joint Commission, 2012; National Hospice and Palliative Care Organization, 2013).

Families are active in serious illness transitions much further upstream, and participate in the care, decision making, and support of their family member long before they enter into hospice (Blum \& Sherman, 2010; Dosser \& Kennedy, 2012; Herbert \& Schultz, 2007; Penrod, et al., 2012). How families make sense of and move through transitions is influenced by cultural beliefs and attitudes, which are shaped by a myriad of factors, including kinship, faith/religion, community, technology, folk systems, formal care systems, and knowledge systems to name a few (Meleis, Sawyer, Im, Hilfinger \& Shumaker, 2000; Leininger, 1988; Whitehead, 2004). Culture informs how families interpret or make sense of transitions, and has been linked to interpretations of prognostic and treatment information, decisions regarding place of care, 
determinations of caregiver roles, and decisions about EOL care (Braun et al., 2008; Bullock, 2011; Donovan et al., 2010; Gunaratnam, 2007).

Views and interpretations of illness and transitions are shaped by cultural factors (Leininger, 1988; Meleis 2010). One cultural factor that may contribute to divergent views of health and illness is education or knowledge, or how individuals, families, or groups come to know information (Leininger, 1988). Cultural clashes between persons with different knowledge systems exist; for example, one study identified divergent viewpoints between lay persons and health professionals. In an attempt to understand perspectives of transitions regarding palliative care and end-of-life care from patients, their family members and clinicians, Reinke et al., (2008) found that clinicians, patients and their families viewed transitions differently. Patients with COPD or advanced lung cancer and their families recognized functional changes as critical events in illness transitions, while their health care providers recognized hospitalizations or changes to medical treatments as critical events in illness transitions (Reinke et al., 2008). Such clashes can contribute to different interpretations of and responses to illness, transitions, and goals of care. Formal medical and/or nursing knowledge and health literacy are possible reasons for divergent viewpoints of illness. Whereas clinicians were well versed in knowledge of diseases, treatment options, and medical terminology, families often lacked this knowledge and were unable to understand complex medical language when trying to come to terms with illness and make difficult care decisions (Ford, Nietert, Zapka, Zoller \& Silvestri, 2008; Herbert \& Schulz, 2006).

Ethno-history is a cultural factor that has been linked to patient and family views of EOL care and the use of advanced directives. When compared to their Caucasian counterparts, persons of African American and Latino descent were found to have different beliefs and attitudes 
toward the use of advance directives, hospice services, and life-sustaining therapies, such as ventilators and feeding tubes (Bullock, 2011; DeSanto-Madeya et al., 2009, Kreling et al., 2010). Furthermore, persons of Dutch, African American, Latino and Hispanic descent had unique beliefs regarding the role of family and religion and/or faith practices at EOL (Bullock, 2011; DeSanto-Madeya et al., 2009; Donovan et al., 2010; Kreling et al., 2010).

Other societal and environmental factors also mediate beliefs about serious illness, particularly EOL care. Environmental contexts, such as place of residence and proximity of family, have been discussed in prior research as significant to family beliefs, values and behaviors in the end stages of illness. Latino immigrants living far away from immediate family navigated EOL care giving differently than Latinos living in their native land or among large family groups (Carrion \& Nedjat-Hajem, 2013). Rural persons living in Canada experienced difficulty accessing specialty palliative services and valued strong community ties in the end phases of illness (Duggleby et al., 2010). Attention to how families rely on a variety of cultural factors to interpret and navigate transitions in serious illness is of utmost importance as failure to successfully navigate transitions in serious illness can and does lead to negative health outcomes.

There is evidence that failure to deliver family-focused, culturally congruent care to family members of persons with serious illness leads to negative health consequences (Csikia \& Martin, 2010; Giesbercht et al., 2012; Herbert \& Schultz, 2006; Paul \& Rattray, 2007). Family caregivers of persons with life-limiting illnesses in the end stages of illness experienced exhaustion, grief, fear and anger when needs were not met and care was delivered in ways that were not compatible with families' understanding, beliefs and values (Khalili, 2007; Braun et al.2008). Moral and ethical concerns manifested as guilt and intrapersonal conflicts in family members of persons in the end stages of illness when they were faced with decisions regarding 
the care of the person and had incongruent understanding of illness, prognosis and treatments with health professionals (Braun et al., 2008; Meeker \& Jezewski,2005; Phillips \& Reed, 2009). Negative psychological consequences extended well beyond the death of the seriously ill family member, and impacted the family member's life for years to come (Paul \& Rattray, 2007). Furthermore, families of ill person who transitioned to formal palliative care or hospice services expressed a desire to "have known about services sooner." Many families enrolled in hospice in the last days or weeks of their relative's illness, and claimed that they were unaware of the services that hospice had to offer (Csikia \& Martin, 2010). Health professionals' failure to discern how families made sense of end of life transitions, and provide culturally congruent, family-focused care resulted in negative health consequences for the patient and family alike.

Cultural factors influence how families make sense of transitions or view health and illness (Leininger, 1988; Meleis, 2010). To gain a better understanding of what cultural factors influenced family transitions to hospice, an observational pilot study was conducted by this investigator (Gallo, 2013). Four hospice admission visits were observed and field notes were recorded, transcribed, and analyzed. Analysis of data revealed that family/kinship, knowledge systems, community/social systems, and faith religion were potentially significant cultural factors in the transition to hospice. Family members described making decisions together and were always the primary caregivers to the hospice patient. When talking with hospice nurses, family members described asking questions of health professionals and trusted family members to get information. Families also described how they knew or didn't know about specialty services, such as hospice, because of past experiences. Family members described how health professionals provided them with information that helped them make sense of the end-stage diagnosis. When nurses asked family members who could help them with respite care, they 
mentioned clergy, extended family, friends, and neighbors. Religion and faith emerged as potentially significant cultural factors as family members described "praying" and stated such things as "only God knows" throughout admission visits. Furthermore, religious artifacts such as Bibles, crosses, and statues were observed in three of the four families' homes.

The aforementioned findings provided a basis for the current study. However, because observations were made in the very end stages of illness, as families transitioned into hospice, the significance of the identified cultural factors further upstream in the illness was unknown. Moreover, because the pilot study was an observational study, questions that further explored the importance of observed cultural factors could not be posed. Previous studies have examined the role of the primary caregiver in the end stages of illness, yet have done little to explicate the experiences of all family members throughout the illness continuum. Furthermore, in existing studies, cultural factors were largely limited to race and ethnicity. It became evident that research was needed that could explore how multiple cultural factors influenced family understanding, behaviors, and decisions in serious illness transitions further upstream in the illness trajectory, beginning at the time of diagnosis.

\section{Significance}

This study adds to the nursing body of knowledge by expanding the inclusion of family beyond primary caregiver, investigating an understudied time period, and describing how specific cultural factors influenced family transitions in serious illness. The majority of prior research has focused on family caregiver transitions in the very end stages of illness, once impending death of the family member was realized and EOL care was initiated (Bourgeois \& Johnson, 2003; Spichiger, 2008; Phillips and Reed, 2009; Csikai and Martin, 2010; Donovan et al., 2010; Duggleby \& Berry, 2005; Duggleby, et al.,2010; Kreling et al., 2010; Waldrop et al., 
2005). Only two studies described family caregiver transitions across the illness trajectory, spanning from pre-diagnosis through bereavement (Edwards, Olson, Koop \& Northcott, 2012; Penrod et al., 2012). These studies focused on the primary caregiver and the ill person, did not extend to other family members, and did not illuminate the role of culture in transitions. Studies of the influence of culture on family decisions in the end stages of illness have been largely limited to race and ethnicity. Very few studies looked at any other cultural factors when determining how families made EOL decisions.

Nurses are charged to provide patient-centered, family-focused care to individuals and families experiencing serious illness (World Health Organization, 2014; The Joint Commission, 2012; National Hospice and Palliative Care Organization, 2013; IOM 2015). In order to help families navigate transitions in serious illness so that they feel confident and secure in their actions, and experience less distress throughout and after serious illness, nurses must be cognizant of how cultural factors influence family transitions in serious illness (Leininger, 1967; Meleis et al.,2000; 2010). Families of seriously ill persons have shared beliefs, actions, norms and lifeways that have been shaped by cultural factors, including religion, kinship, knowledge systems, political systems, and technology to name a few (Leininger, 1988; Whitehead, 2004). Nurses are often the primary caregivers of persons and families going through transitions, and must recognize that long after the ill person is gone it is the family who is left to carry on; families are left to make sense of their world, and their actions and decisions throughout illness well beyond the death of their loved one.

Findings from this study can better prepare nurses to care for families by serving as guides and facilitators of family transitions in serious illness. By understanding how cultural factors influence family transitions in serious illness, nurses can begin to comprehend the 
meaning that families ascribe to events in illness, the reasons why families do what they do, and how families make sense of transitions. Recognizing how cultural factors influence family transitions in serious illness is essential to facilitate transitions in ways that are desired and accepted by families and lessen or alleviate unnecessary distress (Leininger, 1967, 1988; Meleis et al., 2000; Meleis, 2010). In spite of this, little attention has been paid to families as they transition in serious illness throughout the entire illness trajectory, and even less attention has been paid to the influence of cultural factors on family transitions in serious illness.

\section{Purpose}

This study was prompted by a personal experience with serious illness and limited research in the area. While transitioning in illness with a family member, this researcher was challenged to understand how her family made sense of changes that they experienced. This incident led this researcher to wonder "How do others make sense of their experiences?" Looking to the literature, it became evident that little is known about how families make sense of the many changes that they experience from the time that their relative is diagnosed with a lifethreatening illness to the time when they realize that their family members' disease or illness is progressive in nature and enter into EOL care. Even less is known about how cultural factors shape transitions in serious illness, especially in the time span leading up to EOL. Thus, the purpose of this study is to describe from the perspectives of families the influence of specific cultural factors on family transitions in serious illness from the point of diagnosis with serious illness to the acknowledgement that the illness is advanced and will lead to death despite medical intervention. 


\section{Research Question}

The question to be answered by this study is: How do families retrospectively describe the influence of cultural factors in the transition spanning from the point that a diagnosis was made up to the point when the family realized that the family members' disease or illness was progressive in nature, that it could be managed but not cured by medical treatment, and would ultimately result in death?

\section{Definitions}

\section{Transitions}

A transition is a span of time in which change is experienced, has a beginning, middle, and end, and encompasses critical points and events (Meleis et al., 2010). This study focused on the transitional period that began when the family received a diagnosis of serious illness and ended when the family realized that their family members' disease or illness was progressive in nature, that it could be managed but not cured by medical treatment, and that it would ultimately result in death.

\section{Family}

For purposes of this study, family was any person(s) who identified themself as such and were tied to the seriously ill person emotionally, physically or economically (Hanson, 2005)

\section{Serious illness}

Serious illness is a life limiting physical condition in which the ill person experiences gradual physical decline eventually resulting in death (National Hospice and Palliative Care Organization, 2013). Examples of serious illness include: chronic lung disease, chronic heart disease, chronic renal disease, progressive neurological disorders, and various types of cancers. 


\section{Culture}

Culture is the shared beliefs, values, and lifeways of a group that contribute to the meaning ascribed to life experiences. Cultural groups are bound by shared heritage, background, organization, experience, or geographic locale. Culture is shaped by a variety of social systems and environmental elements, otherwise known as cultural factors. Cultural factors include, but are not limited to: technology, religion and philosophy, kinship and society, politics, economics, education or knowledge, and community (Leininger, 1988; Whitehead, 2004). Cultural factors of interest in this study included family/kinship, community/social systems, faith/religion, and knowledge systems.

\section{Family/Kinship}

Family/kinship refers to the hierarchy within the family, expectations and beliefs of the family related to illness, family roles and responsibilities (Leininger, 1988).

\section{Community/Social Systems}

Community/Social systems refers to relationships and interactions with persons outside of the family, such as friends, neighbors, support organizations, employment organizations, health care organizations, and health professionals (Leininger, 1988; Whitehead, 2004).

\section{Faith/Religion}

Faith/religion refers to the belief in a higher deity or power, religious customs and rituals, relationships with religious communities and spiritual or faith leaders (Leininger, 1988).

\section{Knowledge Systems}

Knowledge systems refer to what was known about the illness, what was expected because of that knowledge, how the person came to know, and who the person turned to for answers or advice (Leininger, 1988). 


\section{Method}

Qualitative description using conventional content analysis was used to describe families' retrospective descriptions of the influence of cultural factors in the transition spanning from the point that a diagnosis was made up to the point when the family realized that the family members' disease or illness was progressive in nature, that it could be managed but not cured by medical treatment, and would ultimately result in death. Qualitative description is an appropriate method to use when the purpose of the study is to describe a phenomenon about which little is known (Sandelowski, 2000, 2010). Qualitative descriptions provide full explanations of an experience in the terms of the persons that are living the experience. Qualitative descriptions present the facts, describe the facts in terms of the participants, and convey findings in a meaningful, practical manner (Sandelowski, 2000).

Conventional content analysis was used to analyze data. In this study, data collection was completed using a more deductive approach and was guided by the research question and the cultural factors of interest. Once data was collected, conventional content analysis, which is an inductive approach to data analysis, was employed. Findings were allowed to emerge freely from the data; no preconceived categories are imposed on findings (Hseih \& Shannon, 2005). Conventional content analysis is one of three approaches to qualitative descriptive analysis, and was appropriate to use in this study because little was known about the phenomenon (Hseih \& Shannon, 2005).

\section{Conceptual Framework}

This study was guided by the concepts transitions in illness and culture. A transition is a span of time in which change is experienced. Transitions have an identifiable starting point, where change is first acknowledged, followed by periods of instability, where confusion and 
distress are often experienced (Meleis et al., 2000; Meleis, 2010). Transitions typically end with a new beginning or point of stability. They encompass critical points and events, where individuals or groups develop an increasing awareness of the changes that they are experiencing and often become more engaged in dealing with the transition. Transitions can be facilitated or inhibited by a number of factors, which include personal meanings, cultural beliefs and attitudes, socioeconomic status, and preparation for and knowledge of the change. Therapeutic nursing interventions that take facilitating and inhibiting factors into account should be employed to help individuals or groups master transitions. Mastery of transitions is indicated by individuals developing confidence in coping with transitions, mastering the skills needed to manage the new situation or environment, and adapting to their new situation or environment (Meleis et al., 2000; Meleis, 2010).

Culture is the learned set of beliefs, values and lifeways of individuals, families or groups (Leininger, 1988). Culture informs human behavior and guides thinking, decisions and actions of cultural groups in patterned ways (Leininger, 1988), and contributes to the meaning that individuals, families and groups ascribe to life experiences (Vandenberg, 2008). Experts have challenged how cultural groups are understood and studied and have emphasized that it is not enough to approach culture from only racial and ethnic vantage points (Vandenberg, 2008; Whitehead, 2004). Rather, it is argued that culture is shared among people bound by a common experience; experiences are not limited to race and ethnicity (Vandenberg, 2008, Whitehead, 2004). Such experiences may include geographic locale, education, socio-economic status, and shared life experiences to name a few (Whitehead, 2004; Vandenberg, 2008). Culture is shaped by cultural factors, which include but are not limited to religion, kinship, community, politics, economics, education, folklore, and technology. Each of these factors shape how individuals, 
families, communities and populations behave, understand, and make decisions in relation to life events. These factors are interrelated and give meaning and structural order to the beliefs, values and lifeways of cultural groups (Leininger, 1988; Vandenberg, 2008; Whitehead, 2004).

This study examined how families of persons with serious illness described the influence of four specific cultural factors on transitions in serious illness. Participants in this study were bound by a common experience (serious illness of a relative) and resided within one geographic region. In this study, the transitional time period of interest spanned from the time that participants learned of the diagnosis of serious illness through the time that they realized that in spite of medical treatment, the ill person would not recover from their illness. This point was typically identified with the acknowledgement of EOL. Interview questions were designed to examine how specific cultural factors, such as family/kinship, community/social systems, knowledge systems, and faith/religion, influenced transitions. More specifically, questions were designed to tease out how these factors influenced behaviors, thinking, decisions, and actions throughout illness transitions.

\section{Summary}

Families are integral partners in the care of seriously ill persons and often experience distress while transitioning through serious illness with their relative. Little attention has been paid to families as they transition through illness, particularly prior to the EOL phase. Even less attention has been paid to how families navigate transitions in illness, and what factors influence family behaviors, understanding, and decisions along the way. Though transitions are informed by culture, more specifically cultural factors, little is known about how specific cultural factors influence family transitions in serious illness. The aim of this study was to convey families' retrospective descriptions of the influence of cultural factors in the transition spanning from the 
point that a diagnosis is made up to the point when the family realized that the family members' disease or illness was progressive $\mathrm{n}$ nature, that it could be managed but not cured by medical treatment, and would ultimately result in death. The findings from this study have improved understanding of how cultural factors influenced family comprehension of illness treatments and prognoses, behaviors, and decisions from the time of diagnosis up to the EOL phase. Findings from this study help to prepare nurses to lessen or alleviate unnecessary distress by anticipating family needs and facilitating family transitions in illness. 


\section{Chapter Two: The Literature Review}

Chapter two provides a review of the literature relevant to the proposed study.

Specifically, it reviews the literature related to culture and family transitions in serious illness.

The chapter begins with an explanation of the search process used to select appropriate literature followed by conceptual, methodological and empirical findings from extant literature. It includes a synthesis of findings, including gaps in the current literature and proposed areas for future research. The chapter concludes with a summary, which crystallizes the contents of the chapter.

\section{Literature Search Process}

A systematic review of the literature related to culture and family transitions in serious illness was conducted using the search engines: CINAHL, MEDLINE, PsychINFO, PsycARTICLES, Health and Psychosocial Instruments, and Academic Search Complete. The search term family or caregiver was combined using the connector AND with various combinations of search terms: transitions, EOL, palliative care, hospice, serious illness, chronic illness, end stage illness, and culture. Ancestral and descendent searches were conducted from references of found articles. Limiters narrowed the search to peer reviewed articles written in the English language and published within the last 10 years. Studies were included in the review if they solely focused on families or included families as a component of the study. Articles were excluded from the review if: the ill person was a child, sudden or unexpected death was experienced, or the ill family member suffered from dementia or related illness due to the unique nature of family transitions in these populations. A matrix method was used to organize data (Gerrard, 2011). This matrix can be found in appendix 2.1. 


\section{Conceptual and Theoretical Findings}

Prior studies have provided a foundational conceptual basis for an understanding of transitions in serious illness and culture. Transitions have been defined as change processes that occur over time and have been categorized as developmental, health and illness, situational, and organizational (Meleis et al., 2000; Meleis, 2010). Culture, consistently defined as shared beliefs, values, norms, and lifeways of groups (Leininger, 1988; Vandenberg, 2012), has largely been associated with race and ethnicity. The following sections are dedicated to a discussion of the current conceptual understanding of culture and transitions in serious illness.

\section{Transitions in serious illness}

The existing literature contains conceptual foundations related to individual transitions in serious illness, yet scant literature exists to provide a theoretical understanding of family transitions in serious illness. In her theory of transitions, Meleis (2000; 2010) defines transitions as change processes that occur over time, have an identifiable beginning, middle and end and are marked by critical points and/or events. Persons may experience multiple transitions at once, and transitions may or may not be related. Four distinct types of transitions were identified, and include: developmental, health and illness, situational, and organizational. Developmental transitions relate to normatively governed physical and psychosocial maturations and include birth, adolescence, menopause, aging and death. Organizational transitions refer to changing environmental conditions that affect the lives of clients. Situational transitions are associated with changes in place of care, such as shifts from the hospital to home or admission to a nursing home facility. Other situational transitions identified in the literature include immigration to a foreign country and education transitions, such as moving from an RN to BSN prepared nurse. Health and illness transitions are associated more with experiences and management of chronic 
or serious illnesses, such as congestive heart failure, rheumatoid arthritis, and ovarian cancer. Transitions to palliative care and/or EOL have also been included in this literature (Meleis et al., 2000; Meleis, 2010). Nursing research using Transitions Theory as a framework focused further on care setting transitions, the transition of becoming a new parent, and transitions of family members to supportive roles for cardiac rehabilitation patients. Research that focused on the chronic illness transition specifically centered on the individual experiencing transitions in care locations, transitions in care services, transitions related to specific chronic illnesses, and transitions to EOL or palliative care (Meleis, 2010).

Additional authors have provided definitions of transitions that are consistent with Meleis' theory. In a review of the literature on transitions, Kralik, Visentin, and Loon (2006) found that illness transitions were described consistently as processes that involve multiple changes rather than being discrete events that happen at specific points in time. They also identified conditions that either facilitated or constrained transitions as well as the experience of suffering as a possible catalyst for transitions (Kralik, Visentin, and Loon, 2006). In a review of the literature of key terms used in EOL care, Hui et al. (2014) found the term care transitions was used to signify three distinct type of changes: (1) changes in place of care (2) changes in level of care and (3) changes in goals of care. Changes in place of care refer to changes in settings where care is carried out, such as from hospital to nursing home, from nursing home to home, and so forth. Changes in level of care refer to changes in primary and specialty care providers, such as general practitioners, oncologists, or other specialists. Changes in goals of care refer to the change from curative or disease-modifying treatment to palliative or comfort care measures only (Hui et al., 2014). These changes are consistent with situational transitions and do not elaborate on the health and illness transitions experienced by patients and families at EOL. 


\section{Culture}

The importance of culture in the provision of nursing care to diverse patient populations was first introduced by Madeleine Leininger in 1967. Leininger, both a nurse and an anthropologist, argued that health behaviors could only be changed if nurses and other health care professionals had an in depth understanding of culture; attention to cultural values of persons was just as important as attention to physiologic and emotional needs. Furthermore, Leininger proposed that individuals, families and groups would reject health services that were not congruent with their norms and health values. She defined culture as a learned set of behaviors and beliefs that were related to the background of and societal influences placed on the individual, family, or group (Leininger, 1967). Background and societal influences included such factors as religion and spirituality, politics, technology, education, economics, and environment (Leininger, 1988). These background and societal influences were termed cultural factors (Leininger, 1988).

In literature related to family transitions in serious illness, culture has largely been associated with race and ethnicity. Prior research has sought to determine the relationship of race and ethnicity to specific health care practices among families of persons with serious or advanced illness, such as use of advanced directives, enrollment in hospice, and choice of life sustaining measures such as ventilators and tube feedings (Bullock, 2011; DeSanto-Madeya et al., 2009; Dubenske et al., 2008; Kelley, Wegner \& Sarkisian, 2010; Wright et al., 2013). Additional studies described beliefs regarding use of medical services, dependence on family, and expected roles in end stages of illness among persons with specific racial or ethnic backgrounds (Kreling et al., 2010). This is consistent with research on other health and illness transitions, where investigators looked at the specific experiences of racially or ethnically bound 
cultural groups (Meleis, 2010). Two transition-related studies examined cultural groups other than those bound by race or ethnicity. These studies focused on groups of people bound by place with one study focused on caregivers living in multiple geographic areas in Canada (Giesbrecht et al., 2012) and the other on older adults and families receiving palliative care living in rural Canada (Duggleby et al., 2010).

\section{Methodological Findings}

Quantitative, qualitative, and mixed methods have been used to study family transitions in serious illness; quantitative research has been limited to non-experimental designs that describe family values and/or beliefs at specific points in serious illness (Dubenske et al., 2008) or the relationship of ethno-history to EOL care decisions (DeSanto-Madeya et al., 2009; Kelley, Wegner \& Sarkisian, 2010; Wright et al., 2013). Qualitative methods have been used in research related to family transitions at EOL; a variety of research designs have been utilized to examine different aspects of the phenomenon. Only two qualitative studies were found that examined family transitions across the illness trajectory, yet neither examined the explicit role of culture or cultural factors in transitions. Furthermore, neither of these studies looked at family outside of the primary caregiver role. The following sections discuss the methods used to study family transitions in serious illness, populations studied, and specific aims of previous studies. Studies are categorized as either quantitative/mixed methods or qualitative.

\section{Quantitative and Mixed Methods}

Non-experimental designs have been used to describe relationships among race, ethnicity, or acculturation and EOL care preferences, beliefs, or behaviors. Previous research made comparisons between care preferences of Caucasians and Latinos (Carrion \& Nedjat-Hajem, 2013), Caucasians and African Americans (Bullock, 2011), and Caucasians and Hispanics 
(DeSanto-Madeya et al., 2009). A variety of tools were used to measure acculturation (DeSantoMadeya et al., 2009; Kelley, Wegner, \& Sarkisian, 2010) and communication, treatment, and EOL medical care preferences (Dubenske et al., 2008). Using a mixed methods approach, Bullock described culturally mediated perspectives of EOL and palliative care, and rationale for differences in attitudes and behaviors related to advanced care planning between Caucasians and African Americans (Bullock, 2011). Quantitative analysis of survey data was used to compare preferences of black and white older adults regarding use of advance directives; qualitative analysis of focus group data was done to gain additional insight into the attitudes and beliefs towards the use of advanced directives and death and dying (Bullock, 2011).

Previous quantitative work has represented persons and families receiving care in both community and acute care settings. African American community-dwelling residents over the age of 55 were surveyed to further explore values norms, attitudes and behaviors surrounding EOL decisions (Bullock, 2011). Similarly, elderly Latinos living in Los Angeles were recruited through community senior centers to measure EOL care preferences and attitudes towards three culturally based constructs: family-focused decision-making, patient autonomy, and trust in health care providers (Kelley, Wegner \& Sarkisian, 2010). Ill persons and their families receiving formal care services from acute care medical facilities were represented as well: two studies recruited participants from various cancer care centers in the United States in order to test associations among acculturation scores and treatment preferences, communication, or EOL care (DeSanto-Madeya et al., 2009; Wright et al., 2013).

\section{Qualitative}

A variety of qualitative methods, including phenomenology, qualitative description, grounded theory, and ethnography have been used to study patient and/or family transitions in 
serious illness; particularly at EOL. The majority of studies aimed to explore or describe communication processes among health care providers, patients and families throughout the illness or at specific times in the illness trajectory, such as at EOL (Cskikia \& Martin, 2010; Dalgaard, Thorsell \& Delmar, 2010; Powers, Norton, Schmitt, Quill \& Metzgar, 2011; Wittenberg-Lyles, Goldsmith \& Ragan, 2011). Other studies set out to explore or describe transitions experienced by patients and families at discrete points in the illness trajectory (Donovan et al., 2010; Duggleby \& Berry, 2004; Duggleby et al., 2010; Penrod et al., 2012; Pinnock et al., 2011; Reinke et al., 2008; Tallman et al., 2012; Waldrop et al., 2005). One study aimed to compare the perceptions of transitions in serious illness between healthcare providers, patients and families (Reinke et al., 2008). A few studies examined the role of culture at specific points in the illness trajectory, such as EOL planning or care provision (Bourgeois \& Johnson, 2003; Geisbercht et al., 2012, Kreling et al., 2010; Lockie et al., 2010). Organizational culture, specifically attitudes of health care providers towards integration of palliative care services in the acute care setting, was the focus of one study (Powers, Norton, Schmitt, Quill \& Metzgar, 2011). Populations represented in previous qualitative studies included persons living in rural and urban areas and receiving care in both outpatient and community settings. In the first of two studies in rural settings, older adults receiving palliative care and their families who resided in rural Canada were interviewed to gain an understanding of the transitions they experienced as they moved into palliative care (Duggleby et al., 2010). The second study conducted in a rural locale sought to describe the experiences of rural family caregivers who provided transportation to a seriously ill relative enrolled in palliative care services (Lockie et al., 2010). Urban and metropolitan populations were more widely represented in existing studies. Families and their ill relatives were recruited from urban Canada (Csikia and Martin, 2010), large metropolitan areas 
in the United States (Edwards et al., 2012; Kreling et al., 2010; Reinke et al., 2008; Tallman et al., 2012), and large academic medical centers (Powers et al., 2011) to participate in extant studies. Families and persons receiving care through community services such as hospice, home care, and senior centers were represented in previous work (Csikia \& Martin, 2010; Donovan et al., 2011; Duggleby et al., 2010; Penman, et al., 2013; 2013; Penrod et al., 2012; Waldrop et al., 2005). Likewise, families and persons receiving care in acute hospital settings and specialty care clinics were represented in prior studies (Dalgaard. Thorsell \& Delmar, 2010; Powers et al., 2011; Reinke et al., 2008; Tallman et al., 2012; Wittenberg-Lyles, Goldsmith \& Ragan, 2011).

Families were rarely the explicit focus of previous studies. Rather, families were used as proxies to gain insight into the individual's experience or to gain insight into the EOL caregiving experience. Patient and caregiver dyads were included in studies to describe the significance of identification and explicit communication of the different phases in incurable illness trajectories in a hospital setting (Dalgaard et al., 2010), explore transition experiences of older adults and families receiving palliative care (Duggleby et al., 2010), understand decision-making processes between individuals and family caregivers (Edwards et al., 2012), understand the EOL needs of persons with COPD and informal and professional carers (Pinnock et al., 2011), explore transitions into palliative and EOL care from the perspectives of patients with COPD and lung cancer, their family members, physicians and nurses (Reinke et al., 2008), describe patient and family needs when transitioning to palliative care (Tallman et al., 2012), and determine communication characteristics that influence early palliative care and how they shape patient and family experiences (Wittenberg-Lyles, et. al., 2011). Studies that explicitly included family members of seriously ill persons sought to illustrate how culture shaped attitudes toward dying and practices that assisted them to make sense of their world (Bourgeois \& Johnson, 2003), 
describe perspectives or challenges of family care providers when caring for terminally ill family members (Carrion \& Nedjat-Hajem, 2013; Lockie et al., 2010; Phillips \& Reed, 2009; Waldrop et al., 2005), explore caregiver experiences communicating with health care providers on EOL care options (Csikia \& Martin, 2010; Russ \& Kaufman, 2005), and determine significant cultural factors to family caregivers in the provision of EOL care (Donovan et al., 2010; Kreling et al., 2010).

Most prior research focused explicitly on the transition from curative measures to palliative measures (Csikia \& Martin, 2010; Dalgaard, et al.,2010; Pinnock et al., 2011; Powers et al., 2011; Reinke et al., 2008, Russ \& Kaufman, 2005; Tallman et al., 2012; Wittenberg-Lyles, Goldsmith \& Ragan, 2011), or transitions that occurred after families and patients enrolled in hospice services (Bourgois \& Johnson, 2003; Carrion \& Nedjat-Haim, 2013; Donovan et al., 2010; Duggleby et al., 2010; Kreling et al., 2010; Penman, Oliver \& Harrington, 2013; Phillips \& Reed, 2009; Waldrop et al., 2005). Two studies described transitions across serious or advanced illness trajectories. Using grounded theory methods, researchers developed theory that described the decision making process between family caregivers and persons with advanced cancer (Edwards et al., 2012). In another study, researchers used grounded theory methods to develop a caregiving model across the phases and transitions of EOL caregiving (Penrod et al., 2012).

\section{Empirical Findings}

Existing literature provides limited information regarding family transitions in serious illness and the influence of culture on such transitions. The following section is organized to first share empirical findings that describe family experiences of transitions in serious illness followed by findings that describe family needs and values during times of transition to EOL. 
Next, findings that explicate the different perspectives of families and health care providers throughout transitions in serious illness are provided. Finally, findings are presented that describe what is known about culture and family transitions at EOL. The following headings are used to organize findings: Family transitions in advanced serious illness, family needs and values when entering EOL phase, divergent perspectives of transitions in advanced serious illness, and culture and family transitions at EOL.

\section{Family transitions in advanced serious illness}

Studies have highlighted the many transitions that family members acting as caregivers experience during the end stages of the illness of a relative. Physical and emotional transitions experienced by family caregivers in the EOL phase have been discussed in extant literature, as well as role transitions experienced by family caregivers at EOL. The majority of existing research focused on transitions in the latter stages of illness. Two prior studies discuss transitions across the illness trajectory in an attempt to better understand family caregiver and patient care decisions in the EOL phase. The following section is organized to present what little is known about family experiences of transitions in serious illness using the following subheadings: Physical and emotional transitions at EOL, role transitions at EOL, and decisionmaking and caregiving across life-limiting illness trajectories.

Physical and emotional transitions at EOL. Families experienced health related physical, emotional, and psychological changes in transitions during the advanced stages of their family member's illness. Uncertainty related to prognosis, life-expectancy and available treatment options was experienced by family members acting as caregivers to ill persons (Phillips \& Reed, 2009). Caregivers experienced anxiety and anguish as they witnessed physical and cognitive changes to the seriously ill person, patient suffering at EOL or as they tried to fulfill care giving 
responsibilities throughout the advanced stages of illness (Duggleby et al., 2010; Lockie et al., 2010; Payne et al., 2010; Penrod et al., 2012). Caregivers also had positive emotional experiences while serving as caregiver to the ill person in the latter stages of illness, as many found care giving meaningful and credited it with providing them opportunities to become closer with their dying family member and participate in special life moments (Lockie et al., 2010; Phillips \& Reed, 2009). In addition to emotional and psychological changes, family caregivers also experienced adverse physical changes in the latter stages of illness, including fatigue and exacerbations of underlying illness (Edwards, et al., 2012; Lockie et al., 2010).

Role transitions at EOL. Perhaps one of the most noted and studied experiences of family caregivers transitioning in serious illness was changes in roles and responsibilities, particularly in the late stages of illness. Family caregivers experienced changes to normative behaviors and relationships with the ill person as children became caregivers to parents (Duggleby et al., 2010; Phillips, 2009), or family members had to assume roles and responsibilities that were previously fulfilled by the ill person (Edwards et al., 2012; Waldrop et al., 2005). As illnesses progressed to latter stages, increased responsibilities of caring for the ill person often led to decreased time available for work, school, (Carrion \& Nedjat-Haiem, 2013), social, and family activities (Lockie et al., 2010). Many family caregivers also experienced financial strain due to lost work time or loss of income by either the ill person or the family member acting in the primary care giver role (Carrion \& Nedjat-Haiem, 2013; Waldrop et al., 2005).

Decision-making and caregiving across life-limiting illness trajectories. Studies have described patient and caregiver decision making and caregiving models across life-limiting illness trajectories. Edwards and colleagues (2012) employed grounded theory methods to understand the decision-making process that occurred between a dying individual and his or her 
family caregiver. Findings from this study compared the decision-making process throughout advanced cancer to an intricate dance, where the ill person and their caregiver moved with eachother to gather information and make decisions at different points and times throughout the illness trajectory. The caregiver "entered" the dance prior to the diagnosis of illness, when they encouraged their family member to seek out care for inexplicable symptoms. The caregiver "exited" the dance after the death of their relative. Throughout the illness, the ill person and their caregiver remained engaged with each other to make decisions about care. Over time, as the illness progressed and the ill person was not able to make decisions, the caregiver's decisionmaking responsibility increased. Caregiving responsibilities also increased as the ill person was less and less able to care for him or herself. Caregivers based decisions on previous conversations with the ill person, where the ill person's desires were made known; practical reasons, such as inability to continue to provide care; and communication with health professionals (Edwards et al., 2012).

Penrod and colleagues (2012) used grounded theory methods to explicate a baseline theoretical model of the phases and transitions of caregiving across complex EOL disease trajectories. Persons diagnosed with a variety of life-limiting/terminal illnesses and their family caregivers were represented in this study. Theory emerged from this study that described how family caregivers "sought normal" through the many changes that they experienced throughout the illness trajectory. Caregivers amassed and interpreted information at different points throughout the illness to help them come to terms with the many challenges of caregiving that they encountered. Caregivers sought a state of "normalcy." "Normal" states were achieved when the caregiver felt a sense of "having things under control." The caregiver's ability to interpret and process information along the trajectory was critical to their ability to successfully manage 
complex role demands with each transition and achieve some sense of "normal." Furthermore, because this study looked at persons with a variety of life-limiting/terminal illnesses, the diagnosis of the ill person created variations in caregiving models. Caregivers associated diseases such as HIV and cancer with an abrupt shift from cure to comfort; other diseases were typified by a progressive deterioration and anticipated death; while certain diseases, such as congestive heart failure, followed an unexpected death trajectory and acknowledgement of terminality came late in the illness trajectory (Penrod et al., 2012).

Both studies identified similar phases of transitions experienced by caregivers throughout the illness of their family member. Critical points and events such as changes in treatment priorities, denoted the start and end of transitional phases. For example, obtaining an official diagnosis was noted as the end of one phase and the beginning of another in Penrod and colleagues' (2012) study. Both studies acknowledged multiple phases in illness transitions that began when the ill person experienced symptoms for which medical treatment was sought. This phase ended when a diagnosis of a chronic or life-limiting illness was made, and was followed by a time period in which cure of the disease or remission of the disease was sought. Once patients and families acknowledged that the disease could not be cured or remission could not be maintained, EOL measures and/or services were implemented. This phase concluded with the death of the seriously ill person, when the deceased relative was mourned (Edwards et al., 2012; Penrod et al., 2012). 


\section{Family needs and values when entering EOL phase}

Values and needs of families transitioning in serious illness, particularly in the end stages of illness, emerged as researchers sought to understand communication practices among families and healthcare providers, decision-making processes of families, and experiences of families at different points along the illness trajectory. The following section is organized to further discuss family values related to communication and trust as they entered into EOL care.

Communication. Family needs and values related to communication with health care providers in the latter stages of illness were discussed by a variety of existing studies. Multiple studies reported that in the end stages of illness, families of persons with serious illness valued honest, timely communication from practitioners that was sensitive to individual needs (BernardDubenske et al., 2008; Csikia \& Martin, 2010; Dalgaard et al.,2010; Duggleby et al., 2010; Powers et al., 2011; Tallman et al., 2012; Russ \& Kaufman, 2005). When entering into EOL treatment phases, families of persons with serious illness also valued the use of understandable, non-medical terms when communicating with care providers (Russ \& Kaufman, 2005; Tallman et al., 2012). Family members desired less talk about statistics related to diseases and prognoses and more conversations directed at the specific needs of their family member. Families often became frustrated by healthcare providers who used medical jargon, and stated that they were unable to understand the language used by medical professionals, especially when under duress related to their situation (Russ \& Kaufman, 2005).

Trust. Families valued trusting relationships with physicians and other health care providers. Trusted healthcare providers were able to individualize care to meet the distinct needs and values of the seriously ill person and their family and displayed caring and compassion for the family and their loved one (Duggleby et al., 2010; Powers et al., 2011; Tallman, 2012). Trust 
in healthcare providers was also gained when families received consistent information regarding prognosis, treatment, and available services across the care continuum, and especially at end of life. Families of persons receiving palliative care consultation described feelings of confusion and conflict when they received opposing or conflicting information from care providers (Powers et al., 2011). Similar findings were described by families transitioning to EOL in an acute care setting, who described feeling overwhelmed and confused when health care professionals provided fragmented or inconsistent information related to prognosis and treatment (Russ \& Kaufman, 2005).

\section{Divergent perspectives of transitions in advanced serious illness}

Differences in healthcare provider and patient and family perspectives of advanced serious illness transitions were discussed by two studies. Culture clashes existed between family members and health care providers, which included differences in beliefs and priorities as well as language barriers, increased caregiver burden among family members serving as a caregiver to their dying family member (Giesbrecht et al., 2012). Reinke et al. (2008) supported the idea that cultural differences exist between health care providers and lay persons in their understanding of transitions in advanced serious illness. In a study of persons with COPD or advanced cancer, their family members, and health care providers, Reinke et al., (2008) described how health care providers were more likely to identify hospitalizations and related medical changes as significant transitions in advanced serious illness, whereas patients and families were more likely to identify changes to the ill person's ability to carry out normal daily activities or changes in function as significant transitions. Furthermore, physicians viewed the addition of certain therapies as beneficial, while patients and families viewed the same therapies as just another functional limitation (Reinke et al., 2008). 


\section{Culture and family transitions at EOL}

There is evidence that culture plays a role in family transitions in serious illness, specifically at EOL. Prior research has given most attention to the relationship of race and ethnicity to specific EOL care practices; however, the presence of other social systems and environmental elements has emerged from previous work as well. This section is organized using the following sub-headings to discuss what is known about culture and family transitions in serious illness: ethno-history and EOL decision-making, geographic locale and end stage illness, kinship and serious illness, religion, spirituality and EOL, and rituals and customs at EOL.

Ethno-history and EOL decision-making. Relationships between race and ethnicity to EOL care preferences and advanced care planning were described by three separate studies (Bullock, 2011; DeSanto-Madeya et al., 2009; Kreling et al., 2010). Comparisons of persons of African American, Hispanic, and Latino descent to their Caucasian counterparts revealed that Caucasians were more likely to complete advanced directives, choose less aggressive treatments, and use hospice services than persons of African American, Hispanic, or Latino descent, respectively (Bullock, 2011; DeSanto-Madeya et al., 2009; Kreling et al., 2010). African Americans expressed a fear of having treatments withheld if advanced directives were completed, and therefore wished to rely more heavily on family discussions with health care providers to see that EOL needs would be met. Additionally, African Americans believed that God would guide health and that completing advanced directives might interfere with the chance of a miracle occurring (Bullock, 2011). In regard to using hospice services, white non-Latinos typically had a prior knowledge of hospice, whereas Latinos had no knowledge or held misconceptions about hospice services before enrollment. Further contributing to the decision to 
use hospice services among the two groups were cultural beliefs related to discussions about illness and death. Latinos preferred secrecy about prognosis and often wanted to make decisions for the patient, rather than involve the ill person in decisions (Kreling et al., 2010).

Geographic locale and end stage illness. In addition to race and ethnicity, the significance of geographic locale to specific transitions in serious illness has been described in existing literature; where families and patients lived determined access to services, availability of supportive family members, and commute times to care providers (Giesbrecht et al., 2012). Persons who lived in rural areas did not have palliative care specialist in their area and had to travel long distances to obtain specialist services (Duggleby et al., 2010). Long commutes to specialty care providers created an additional burden on family caregivers, as commutes resulted in lost time for work, and personal exacerbations of illness. Poor road conditions, inclement weather, traffic accidents and breakdowns often made travelling long distances more difficult (Lockie et al., 2010). In rural areas where jobs were scarce and young persons often moved away to pursue work and other opportunities, families saw a decline in available supportive persons living in close proximity (Giesbrecht et al. 2012).

Kinship and serious illness. Findings from several studies highlighted the importance of kinship, or family, to transitions in the end stages of serious illness. Families valued inclusion in health care conversations with health professionals and care decisions throughout the disease trajectory (Bullock, 2011; Csikia \& Martin,2010; Kelly, Wegner \& Sarkisian,2010; Kreling et al., 2010; Russ \& Kaufman, 2005). Families from a variety of cultural backgrounds felt obligated to care for their seriously ill family member, especially at the end stages of illness. Additionally, when providing care in the end stages of illness, families were often reluctant to 
seek help from outside sources (Bullock, 2011; DeSanto-Madeya et al., 2009; Donovan et al., 2010).

Religion, spirituality and EOL. Religion and spirituality were significant to families transitioning in serious illness, particularly in the end stages of illness. Religious beliefs increased family caregiver's confidence in decisions in the end stages of illness (Donovan et al., 2010). Religious communities and religious leaders were sometimes turned to for comfort, support and advice by families transitioning at the very end stages of illness (Bullock, 2011; Waldrop et al., 2005). Families eligible for palliative care services valued spiritual support that promoted a sense of meaning and balance (Tallman et al., 2012), and engagement in religious practices, such as praying or sharing a meal, allowed family caregivers to share meaningful experiences with their loved one in the final stages of illness (Penman et al., 2013). Only one study alluded to negative implications of religion and spirituality at EOL. In this study, researchers found that hospice family caregivers' faith was challenged when the illness became overwhelming or death was imminent (Donovan et al., 2010). Among this group, researchers also found that faith and religious beliefs decreased futile treatments and increased quality of life measures in the end stages of illness (Donovan et al., 2010).

Rituals and customs at EOL. Only one study described the importance of cultural rituals to family transitions at EOL. Through story-telling, family members of ill persons approaching death described participation in ritualistic events with their loved ones that symbolized approaching death and provided closure and spiritual comfort to both themselves and their dying family member. This study highlighted the importance of integrating cultural practices into the care of families transitioning in serious illness (Bourgeois \& Johnson, 2004). 


\section{Synthesis}

Families are important care partners in serious illness, yet little attention has been paid to how families make sense of transitions across the illness trajectory. In fact, the majority of existing studies did not have an explicit focus on families or only considered the transitions that family caregivers experienced as they entered into the very end stages of illness. Findings from prior research identified that family caregivers interpreted the meaning of transitions in the end stages of illness differently than medical providers; however, there is not a clear understanding of how culture, more specifically, cultural factors contributed to families' interpretations of transitions across the illness trajectory. No studies examined the influence of culture on family transitions during earlier stages in illness. Furthermore, little is known about how culture influences family transitions outside of racial and ethnic differences at EOL.

In studies most closely related to this work, models of caregiving (Penrod et al., 2012) and the decision making process between the caregiver and the person with advanced cancer (Edwards et al., 2012) were described. Within their findings, Penrod and colleagues (2012) and Edwards and colleagues (2012) described phases of patient and caregiver transitions from prediagnosis to bereavement. While these studies provided a broader view of the illness trajectory than the majority of prior studies, they remained focused on direct family caregivers. Neither study explored family transitions outside of the primary caregiver role. Furthermore, these studies did not fully explicate the many factors that could influence caregiving and decisionmaking throughout the illness trajectory.

This study provides a broader view of transitions in serious illness by focusing on an understudied time period; the time beginning when the family received a diagnosis of serious illness to the time when the family member acknowledged the poor prognosis and imminent 
death of their relative. This study does not extend into the provision of EOL care. Rather than focus on only the caregiver, this study expands inclusion of family to any involved family member of a person with serious illness, and therefore provides a more extensive view of the entire family's experience. This study looks at how family/kinship, knowledge systems, community/social systems, and faith/religion influenced family transitions in serious illness. This study describes how and when these cultural factors come into play throughout the illness trajectory, and moves beyond previous work by explicating how these factors influence family understanding, behaviors, and decisions throughout the illness trajectory. A full description of findings is included in chapter four. Table 2.1 provides a brief overview of what is known from previous research and what this study adds.

Table 2.1 What is known vs. what this study adds

\begin{tabular}{|c|c|c|}
\hline What is known & & What is added \\
\hline$E O L$ & $\begin{array}{l}\text { Pre-Diagnosis through } \\
\text { Bereavement }\end{array}$ & Diagnosis up to EOL \\
\hline 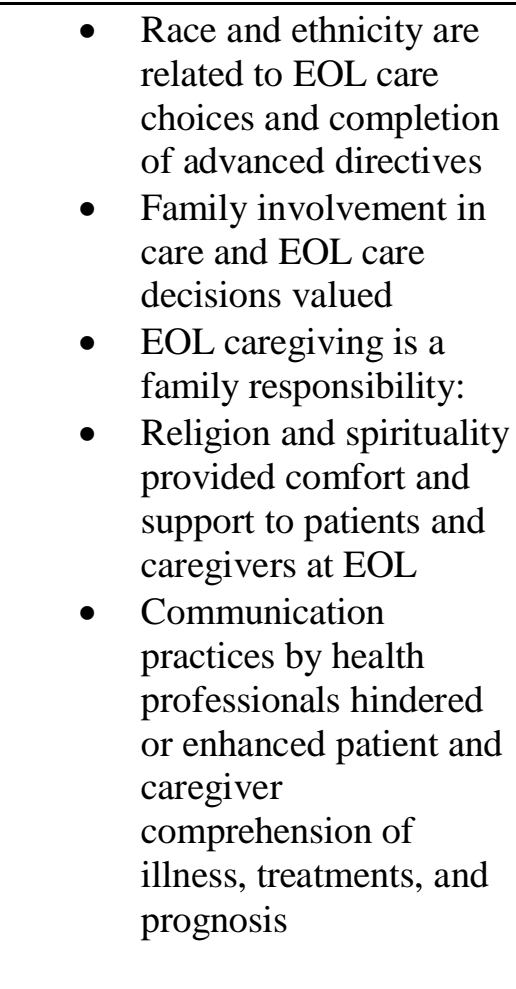 & $\begin{array}{l}\text { Phases of transitions } \\
\text { from pre-diagnosis } \\
\text { through bereavement } \\
\text { identified } \\
\text { - Models of caregiving in } \\
\text { advanced illness } \\
\text { described } \\
\text { - Successful management } \\
\text { of events throughout } \\
\text { transitions was impacted } \\
\text { by caregivers' ability to } \\
\text { interpret and understand } \\
\text { events and information } \\
\text { Caregiver and ill person } \\
\text { decision making process } \\
\text { described } \\
\text { Treatment phase of } \\
\text { transitions was hindered } \\
\text { by barriers within the } \\
\text { health care system } \\
\text { Desire for ill person to } \\
\text { retain decision-making }\end{array}$ & $\begin{array}{l}\text { Focus on persons bound } \\
\text { by shared experience } \\
\text { - Focus on understudied } \\
\text { phase from diagnosis up } \\
\text { to EOL } \\
\text { - Description of the role } \\
\text { of cultural factors on } \\
\text { family transitions } \\
\text { Expansion of family to } \\
\text { include all involved } \\
\text { family, not just primary } \\
\text { caregiver }\end{array}$ \\
\hline
\end{tabular}




\begin{tabular}{|c|c|}
\hline 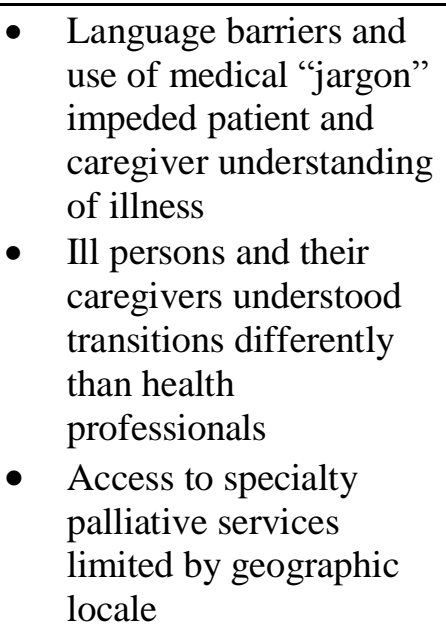 & $\begin{array}{l}\text { authority as long as } \\
\text { possible }\end{array}$ \\
\hline
\end{tabular}

Examination of the influence of family/kinship, knowledge systems, community/social systems, and faith/religion on family transitions in serious illness provides a better understanding of how cultural factors influenced family transitions across the illness trajectory rather than just in the EOL phase. More specifically, findings from this study describe how these factors influenced family members' behaviors, understanding, and decisions at throughout serious illness transitions. By understanding how cultural factors influenced family transitions in serious illness, nurses can begin to comprehend the meaning that families ascribe to events in illness, the reason families do what they do, and how families make sense of transitions. This understanding will allow nurses to promote healthy transitions in serious illness by providing culturally congruent, supportive, patient-centered and family-focused care to families across the entire illness trajectory, rather than just at the end of life.

\section{Summary}

Chapter two presented a review of the literature related to culture and family transitions in serious illness. A description of the literature search process was provided. Conceptual and theoretical findings related to culture and family transitions in serious illness were presented. Methodological findings, which included quantitative, qualitative, and mixed methods, were 
discussed. Empirical findings were presented that described family experiences of transitions in advanced serious illness, family needs and values when entering EOL, conflicting family and healthcare provider perspectives of transitions in advanced serious illness, and culture and family transitions in EOL. A synthesis of findings that summarized what is known about the phenomenon and gaps in the literature concluded the chapter. 


\section{Chapter 3: Methodology}

This chapter discusses the methodology and analytical techniques used to answer the research question - How do families retrospectively describe the influence of cultural factors in the transition from the point when a diagnosis of serious illness was made up to the point when the family realized that the family members' disease or illness was progressive in nature, that it could be managed but not cured by medical treatment, and would ultimately result in death? Qualitative description is an appropriate method to use when the purpose of the study is to describe a phenomenon about which little is known (Sandelowski, 2000). This retrospective qualitative descriptive study employed conventional content analysis as an analytical tool to describe north central WV descriptions of the influence of cultural factors on transitions in serious illness; spanning from the time when a diagnosis was received up through the time when families realized that their family members' disease or illness was progressive in nature, that it could be managed but not cured by medical treatment, and that it would ultimately result in death. Family members of persons with the following illnesses were represented in this study: various types of cancer, aortic stenosis, congestive heart failure, and Parkinson's disease. Chapter three is organized using the following headings: philosophical underpinnings, sampling and recruitment, data collection, data analysis, methods to assure rigor, and human rights considerations.

\section{Philosophical Underpinnings}

\section{Qualitative Description}

Qualitative description was used to answer the following research question for this study: How do families retrospectively describe the influence of cultural factors in the transition spanning from the point that a diagnosis was made up to the point when the family realized that 
the family members' disease or illness was progressive in nature, that it could be managed but not cured with medical treatment, and would ultimately result in death? Qualitative description falls under the naturalistic paradigm, where researchers study phenomena in their natural setting with the philosophical stance that phenomena are bound by time and context (Tavakoi \& Zeinaloo, 2004). Naturalistic inquiry is appropriate to use when little is known about a phenomenon. In qualitative research, participants are purposefully selected to produce information rich data for analysis and ultimately to achieve the purpose of the study. Data, typically gathered through interviews, observations, focus groups, and/or artifacts, are analyzed inductively to allow findings to freely emerge (Tavakoi \& Zeinaloo, 2004).

Qualitative description is most used to determine the "who, what, and where of events (Sandelowski, 2000)," and provide rich, thorough explanations of an experience of the persons that are living the experience. "The qualitative descriptive study is the method of choice when straight descriptions of phenomena are desired (Sandelowski, 2000, p 339).” In qualitative descriptions, researchers are most interested in gaining candid answers to questions of special relevance, unbound by pre-existing theoretical or philosophical commitments. Unlike other naturalistic methods, language is considered not merely an "interpretive structure that must be read but the vehicle of communication itself (Sandelowski, 2000, p 336).” Investigators using qualitative description strive to get the facts, correctly describe the facts as perceived by the participants, and then convey findings in a manner that is logical and practical (Sandelowski, 2000).

Design features of qualitative descriptions are consistent with naturalistic inquiry. Sampling is done using purposeful techniques, where participants are chosen to obtain rich data for the purposes of the study. Data collection is done through semi-structured interviews with 
individuals or groups, observations, and/or examination of artifacts or records. In this study, the interview guide used for data collection was developed based on the research question and the cultural factors of interest. Analysis of data was completed using a technique known as qualitative content analysis (Sandelowski, 2000).

\section{Conventional Content Analysis}

Qualitative content analysis can be done using either a deductive or inductive approach. Data analysis was completed using conventional content analysis, which is an inductive approach. It is typically used when the aim of a study is to describe a phenomenon, and existing theory or research on the phenomenon is limited (Hsieh \& Shannon, 2005). This inductive approach was used to analyze data: findings were allowed to naturally emerge from the text Conventional content analysis was a good fit for data analysis in this study because little was known about the influence of cultural factors on family transitions in serious illness. Codes emerged from the data and were moved up the ladder of abstraction, and then grouped into higher order categories so that the phenomenon could be better understood and new knowledge generated (Elo \& Kyngas, 2007). Findings were organized to answer the research question and address the cultural factors of interest.

\section{Sampling and Recruitment}

\section{Sampling}

Purposive sampling, or the careful selection of participants who have a particular knowledge or experience with a phenomenon (Polit \& Beck, 2014) was used in order to obtain cases that were information-rich in relation to the purpose of the study (Sandelowski, 2000). Participants were chosen who experienced the loss of a family member due to serious illness. Serious illness included diseases in which gradual physical deterioration resulting in death was 
experienced. For purposes of this study, participants were included if their family member died as a result of any serious illness. Family members of persons with the following serious illnesses were represented in this study: various types of cancer, aortic stenosis, congestive heart failure, and Parkinson's disease. Consistent with qualitative description and content analysis, the intent of this study was to gain insight into family perspectives of transitions in serious illness, a phenomenon about which little is known (Hsieh \& Shannon, 2005; Penrod et al., 201; Reinke et al., 2008). Data collection ceased once data saturation occurred and themes and categories became repetitive and redundant, and the researcher decided that no new information could be gleaned by further data collection (Polit \& Beck, 2013).

\section{Inclusion Criteria}

To be included in the study, participants had to be a bereaved family member of an adult person who died from serious illness within the past three years, over the age of 18 , and willing to participate in the study. Serious illness was denoted by the presence of a life-limiting physical condition in which gradual physical decline that eventually resulted in death was experienced. Family members of persons with the following illnesses were represented in this study: various types of cancers, aortic stenosis, congestive heart disease, and Parkinson's disease. It should be noted the original time frame for death to interview was one year. However, once recruitment began, multiple persons approached the researcher and stated that it would be too difficult for them to talk in depth about their relative within the first year of bereavement. No best practices on appropriate time frames to interview bereaved family members could be found, however, input was sought from two experts in palliative care and family research, and it was determined that within three years of death was a reasonable time frame for the inclusion criteria of this study. Furthermore, it was argued by these experts that significant life events are remembered 
regardless of the time elapsed. Three additional experienced researchers were consulted about the appropriateness of expanding this time-frame and agreed that within three years of death was reasonable for inclusion criteria.

\section{Exclusion Criteria}

Participants were excluded if they were non-English speaking or under the age of 18. Family members of persons who died of sudden or acute illness or from Alzheimer's disease or similar neuro-cognitive disorder were excluded from the study as literature suggests that such persons have distinct experiences.

\section{Recruitment}

The researcher had good working relationships with several community agencies within the region that facilitated recruitment. Participants were recruited through a local senior citizen center, two church-affiliated bereavement groups, and by word of mouth. Other bereavement groups and organizations were approached for recruitment, however no participants were obtained from these organizations. Several participants were recruited by word of mouth through personal friends and colleagues of the researcher. Fifteen persons were recruited for this study. Two recruits did not meet inclusion criteria. One recruit opted out of participating in the study.

During recruitment, the following procedures were used (1) the investigator provided an overview of the study and inclusion and exclusion criteria to all key contact persons (friends, colleagues, nurse leaders, organizational leaders); (2) informational letters and recruitment flyers were provided to all key contact persons; (3) the researcher provided a verbal overview of the study and inclusion and exclusion criteria was given to groups that contained potential participants by the investigator; (4) flyers advertising the study were distributed to community 
agencies such as the local senior center and bereavement groups to display on bulletin boards and in informational newsletters; (5) flyers advertising the study were distributed during group meetings in which the investigator provided a verbal overview of the study and inclusion and exclusion criteria; (6) interested participants were asked to contact the investigator using the contact information provided on the flyer; (7) initial telephone conversations included a brief introduction of the researcher, an overview of the purpose, benefits, risks, and confidentiality of the study. The investigator also reviewed inclusion and exclusion criteria, and then scheduled a mutually agreed upon time and place to meet with the participant to conduct the interview; (8) The interview meeting began with a re-iteration of participant rights and purpose of the study; all participants also received a letter explaining the purpose of the study, participant rights, and researcher and IRB contact information. Interviews were conducted, at the end of which participants were asked if they knew of anyone else who had gone through a similar experience and may be interested in participating in the study, (Polit \& Beck, 2014).

Two participants were recruited using this snowball approach. A total of 15 persons were recruited for the study, however, two recruits did not meet inclusion criteria and one recruit declined after the initial phone conversation. All recruited participants were asked if they would like to include other family members in the interview, however, only one participant agreed to this and included her husband, who was the son of the deceased. All other participants declined stating that other family members could not be available because of difficulty scheduling time to meet together, living far distances from family members, or claimed that it would be too emotionally difficult or uncomfortable to include other family members. 


\section{Data Collection}

Data were obtained through digitally recording in-depth, semi-structured, retrospective interviews with family members of persons who died within the past three years of serious illness. One interview with each participant was conducted; all participants agreed to follow-up contact if needed to clarify statements or obtain additional information. A follow-up interview was conducted with one participant. This interview was conducted after the researcher re-read the transcript from the initial interview and determined that additional information was needed. The follow-up interview was completed at the participant's place of employment. During this interview, questions were asked to clarify statements made during the initial interview, such as at what specific points during the illness family conversations occurred and when decisions were made. Initial interviews with all participants were conducted face-to-face in a quiet, private location that was mutually agreed upon by the researcher and participant. Four interviews were conducted in participants' homes, three were conducted in participants' places of employment, two were conducted in participants' churches, one was conducted at the local senior center, and one was conducted in a private meeting room at a local library. One interview was conducted with two family members.

The first interview was conducted on $2 / 4 / 15$ and the last was conducted on $4 / 14 / 15$. No two interviews were conducted on the same day, and the researcher took measures to guard against bias and interviewer fatigue. A journal was kept throughout the research process, and after each interview this researcher reflected on and journaled impressions and thoughts about interviews and participants. Furthermore, this researcher would discuss impressions of interviews and participants with a qualified researcher to gain opinions and insights. Interviews ranged from 8 to 55 minutes in length with the median length of 30 minutes. Although one 
interview was notably shorter in duration than others, valuable information that addressed the research question was still obtained from the transcript. The researcher noted that this participant seemed slightly uncomfortable during the interview, and did not elaborate on several of her responses. This particular participant was also asked to participate in a follow-up interview, but never responded to the researcher.

During all interviews, the researcher clarified data by summarizing key statements of participants, verifying summations, and asking if the participant would like to add anything else. Furthermore, member checks were done with three participants to make sure that findings truly reflected the descriptions that were provided. Select demographic data was collected on participants and included the participant's age, relationship to deceased, age of deceased, medical diagnosis of deceased, and time since death.

\section{Interview Questions}

In keeping with naturalistic inquiry, semi-structured interviews using open-ended questions were conducted to elicit retrospective family descriptions of how cultural factors influenced transitions from the point of diagnosis with serious illness to the acknowledgement that the illness was advanced and would lead to death despite medical interventions. A copy of the interview guide can be found in appendix 3.1. Development of interview questions was informed by the research question and associated concepts of transitions in illness (Meleis, et al., 2000), phases of transitions in serious illness (Penrod et al, 2012; Edwards et al., 2012), cultural concepts, including kinship, faith and/or religion, community/social systems, and knowledge systems (Leininger,1988; Vandenberg, 2008; Whitehead, 2004), and observations from a pilot study of families transitioning to hospice conducted by this researcher (Gallo, 2013). 
The time span for this particular study was informed by work done by Penrod et al. (2012) and Edwards et al. (2012), which identified stages of transitions experienced by caregivers of persons with serious illness ranging from pre-diagnosis to bereavement. Interview questions focused on the time spanning from the point that a diagnosis with a serious illness was made up to the point when the family realized that their family members' disease or illness was progressive in nature, that it could be managed but not cured by medical treatment, and that it would ultimately result in death This time span was selected because the majority of prior research has focused on the very end stages of illness, after the family realized that additional medical care would be futile and entered into EOL care (Bourgeois \& Johnson, 2003; Spichiger, Phillips and Reed, 2009; Csikai and Martin, 2010; Donovan et al., 2010; Duggleby \& Berry, 2005; Duggleby, et al.,2010; Kreling et al., 2010; Waldrop et al., 2005).

Questions were designed to gain family perspectives of transitions and identify how cultural factors shaped transitions. A recent pilot study conducted by the investigator hinted at cultural factors that influenced family transitions to hospice (Gallo, 2013). Observations of families during initial hospice admission visits revealed that family relationships, or kinship, were potentially significant to the transitions into hospice care. Other cultural factors that were evident in observations included faith and/or religion, community, formal care systems, and knowledge systems. Questions were designed to further explore these elements: kinship, or family relationships, roles and responsibilities; faith and religion, or supernatural beliefs and practices; community relationships, or the interwoven connections formed within the society in which one lives, works, or participates, including relationships and interactions with formal care providers and health care systems; and knowledge systems, which include what the family knew 
about the illness, diagnosis, prognosis, treatments and care practices, and how they came to know it.

\section{Data Management}

Data management included a multi-step process. Digitally recorded interviews were transcribed by a professional transcriptionist who is familiar with HIPAA (Health Information Portability and Accountability Act) regulations and who signed a confidentiality contract. The investigator reviewed all transcripts word by word while simultaneously listening to the recordings to ensure accuracy. Transcripts were de-identified by removing all participant names, family member names, references to other individuals, references to particular health professionals, and references to health care systems. Once transcripts were evaluated for accuracy, they were deleted from the audio recorder. Transcripts were entered into ATLAS.ti version 7.0.83 where data were organized, managed and manipulated.

\section{Data Analysis}

Data were analyzed to answer the research question: How do families retrospectively describe the influence of cultural factors in the transition spanning from the point that a diagnosis with serious illness was made up to the point when the family realized that family members' disease or illness was progressive in nature, that it could be managed but not cured by medical treatment, and would ultimately result in death? The researcher was specifically looking for the presence of cultural factors that informed family transitions in serious illness. Data analysis followed a multi-step process; (1) Eleven transcripts were read in their entirety multiple times in order for the investigator to fully grasp the totality of the phenomenon; (2) The investigator made notes of first impressions, thoughts and initial analyses in a written journal; (3) Data was re-read and the investigator highlighted sections of the text that provided insight into the research 
question; highlighted sections were transferred onto a new document so that they could be further analyzed; (4) Text was read word by word to derive codes; initial codes were data near in order to reflect true participant descriptions; (5) From the initial codes, 43 more abstract codes materialized that depicted more than one critical idea; (6) These codes were sorted into 19 higher level categories that depicted more abstract themes and ideas; this process continued until no higher level could be achieved, resulting in seven final themes with five related sub-themes; (7) Descriptors for each theme were developed (Elo \& Kyngas, 2007; Hseih \& Shannon, 2005). At each step of the process, data was reviewed and assessed by a second, highly qualified qualitative nurse researcher.

\section{Methods to Assure Rigor}

The investigator undertook measures to assure credibility and trustworthiness of findings. A major threat to the credibility and trustworthiness of conventional content analysis is: “...failing to develop a complete understanding of the context, thus failing to identify key categories. This can result in findings do not accurately represent the data (Hseigh \& Shannon, 2005, p. 1280)." To combat this, the investigator was attentive to four quality criteria: credibility, dependability, confirmability and transferability (Polit \& Beck, 2014).

\section{Credibility}

Credibility, or "confidence in the truth value of the data and interpretations (Polit \& Beck, 2014, p. 323)," was established through careful research design and adherence to established research procedures. The research question emerged from personal experience, existing theory, and prior research. The research method was chosen based on appropriateness to answer the research question. The research proposal was evaluated and approved by a committee of expert qualitative researchers. The investigator continually referred to the 
committee chair and committee members throughout the study as needed to collaborate on research procedures. Additionally, this investigator protected from bias by identifying personal feelings on the subject matter prior to beginning the study and maintaining awareness of personal thoughts and feelings throughout the study.

\section{Dependability}

The researcher assured dependability, or "the stability of data over time and conditions (Polit \& Beck, 2014, 323)," by adherence to study design procedures. Inclusion and exclusion criteria were evaluated on all participants; any participants not meeting criteria were excluded from the study. Additionally, all participants were asked the same interview questions, though prompts were used to elicit additional information as it emerged from the interviews.

\section{Confirmability}

Confirmability, or the "maintenance of objectivity in the study (Polit \& Beck, 2014, 323)," was established by presenting findings using the language of the participants. During interviews with participants, the investigator summarized statements and confirmed summations with participants. Furthermore, member checks were completed with three participants to ensure findings adequately reflected their descriptions. Additionally, during analysis of findings, confirmability was assessed through use of a second experienced qualitative researcher (committee chair) independently coding random selections of transcripts and comparing findings to those of the original researcher (Polit \& Beck, 2014).

\section{Transferability}

Transferability, or the "extent to which findings can be applied to other settings or groups (Polit \& Beck, 2014,323)," was addressed by presenting findings using rich descriptions. Furthermore, a rich description of study participants was included. Descriptions of participants 
allow health professionals to better understand the compositions of families participating in the study and the types of illnesses that families were experiencing so that they can compare those characteristics and circumstances with the families that they serve. Health care providers providing care to similar populations may be able to apply findings from this study to their individual practice. Health care providers from dissimilar populations may gain insight into how cultural factors might influence family transitions in serious illness.

\section{Human Rights Considerations}

IRB approval was obtained by West Virginia University; attention to participant autonomy, beneficence, and the benefits and risks of participation in the study was maintained by the researcher. Recruitment of participants began only after IRB approval was granted. This was an exempt study as it met criteria for category two exemption as outlined by West Virginia University. Exempt research is: "Research involving the use of educational tests (cognitive, diagnostic, aptitude, achievement), survey procedures, interview procedures or observation of public behavior (West Virginia University, 2015, 41)." Data were obtained through interviews, no participant identifiers were kept on interview records, and disclosure of participant responses did not place subjects at risk of criminal or civil liability or were not damaging to the participant's financial standing, employability, or reputation. Prior to participating in the study, participants were provided with a written description of the study. Participants were informed of their rights as a research participant, including their right to cease participation at any time. A written letter explaining the participant's rights was given to each participant. A copy of this letter can be found in appendix 3.2. Participation in the study was kept confidential. Unique identifiers were given to study participants and all transcripts were cleaned of participant names, health care provider names, and any other personal identifiers prior to analysis. Transcripts and 
demographic information were kept in a password secured computer. No hard copies of transcripts and demographic forms were kept. Pseudonyms were used to report findings from the study.

As an exempt study, risks to participants was minimal. Participants may have benefitted from being in the study as previous researchers found that even when emotional distress was experienced, bereaved family members reported satisfaction with participation in studies. Bereaved family members stated that it felt good to help others and that sharing their story was cathartic (Koffman et al., 2011). The benefit to society may occur through a greater understanding of the role of culture on family transitions in serious illness; which may in turn lead to improved therapeutic care interventions for families throughout the illness trajectory.

The risk of studying family transitions in serious illness to participants was, like other bereavement studies, psychological distress (Rosenblatt, 1995). All efforts were made to ensure that no harm came to participants in this study. Participants were informed of potential risks of involvement prior to agreeing to participate in the study. Participants were made aware that questions may arouse feeling of sadness and could be stressful for them (Koffman et al., 2011). Because reminiscing about a deceased family member can provoke feelings of sadness, the investigator was cognizant of signs of emotional distress during interview periods and planned to offer cessation of the interview and referral to professional psychological, psychiatric or bereavement services if deemed necessary (Stroebe, Stroebe \& Schut, 2003). The investigator has been a registered nurse for 17 years, has experience working with bereaved family members, and is knowledgeable of signs of emotional distress and complicated grieving. No participants demonstrated signs of emotional distress during the interview, however, since emotional distress may have been experienced after the interview session as well, all consenting study participants 
were given a hard copy list of available professional psychological, psychiatric and bereavement services within the geographic area of the study.

\section{Summary}

Chapter three described the methods that were undertaken to complete this study. Philosophical underpinnings of qualitative descriptive methods and conventional content analysis were discussed. Consideration for human rights was addressed. Purposeful and snowball sampling measures were described, and inclusion and exclusion criteria for the study was outlined. A multi-step recruitment process was discussed. Data collection using in-depth, retrospective interviews comprised of semi-structured questions was discussed. Analysis of the data using a multi-step inductive analytical process was described. The chapter concluded with a description of measures that the author adhered to in order to ensure credibility and trustworthiness of findings. 


\section{Chapter 4: Findings}

Within chapter four are findings of this qualitative study of families' retrospective descriptions of the influence of cultural factors on transitions in serious illness. Specifically under investigation was the time period spanning from when families first learned of the illness diagnosis up to the point when the family realized that the family members' disease or illness was progressive $\mathrm{n}$ nature, that it could be managed but not cured by medical treatment, and would ultimately result in death. Contents of this chapter include a description of the participant families and a report of findings, which includes direct quotes to support the abstraction of categories. Pseudonyms are used throughout this narrative to protect participant and family identities. The following headings are used to organize this chapter: description of participants and study findings. Study findings are further organized into section headings which are titled to address the research question and are as follows: Descriptions of the influence of kinship on family transitions, descriptions of the influence of knowledge systems on family transitions, descriptions of the influence of community/social systems on family transitions, descriptions of the influence of faith and religion on family transitions. Under each heading are themes and subthemes that describe study findings.

\section{Description of Participants}

Twelve persons representing eleven bereaved families participated in this study. One family was represented by a husband and wife; the husband lost his mother to Parkinson's disease 26 months prior to the interview. These two participants were interviewed together, at their request. Throughout the interview, they went back and forth with answers, and supported and elaborated on each other's statements. Additionally, the participants' non-verbal behaviors suggested mutual agreement with each other's narrative. Although given the opportunity for 
multiple family members to participate in the interview, the remaining families were represented by only one family member. Participants stated that it was too difficult to schedule time to meet with additional family members, family members were not interested in participating in the study, family members did not live close, or the participant did not feel comfortable interviewing in the presence of additional family members.

Participants in this study were related to the ill person in a variety of ways. The majority were biologically or legally related to the ill person, and included daughters (2), nieces (2), sons (2), husband (1), sisters (2), granddaughter (1), and daughter-in-law (1). Although biologically related to the ill person as a niece, one participant identified the ill person as her father, stating "they raised me the majority of my life." One participant did not claim a biological relationship with the deceased, yet identified himself as a close friend, describing the close bond that they shared by stating: "Once in a lifetime. You know, your special friend. What's the word?..The soulmate." Participants described complex family compositions which included traditional nuclear families, blended families, extended families, estranged families, and families that were bound by something other than biologic relationships. For example, one of the more complex families was comprised of a middle-aged ill male, his brother, sister-in-law, two adult nieces, and his adult daughter and son. An estranged wife was mentioned by this family, but she did not play a role in direct care, supportive care, or care conversations throughout the illness transition. The ill male eventually moved in with his brother and sister-in-law, and they became his primary caregivers as the illness progressed.

Participants identified with a variety of roles within the family. Six of the twelve participants self-identified as a primary caregiver and/or decision maker for the ill person throughout the illness, while the remaining six participants identified having played a more 
supportive role throughout the illness. In this study, participants identified spouses, children, nieces, and siblings as primary caregivers to the ill person. Additionally, participants identified in-laws, cousins, siblings, children, step-children, nieces, grandchildren, and close friends as being involved in and providing support throughout transitions. The majority of participants described families that had four or more involved members throughout illness transitions. Involved family members were described as living in close proximity to the ill person and living great distances from the ill person. The table located in appendix 4.1 illustrates demographic information of study participants, including age, gender, and relationship of participant to the deceased, as well as select information of the deceased.

All participants were persons living in north central WV who had lost a family member as a result of serious illness within 3 to 35 months of the interview. Participants ranged in age from 28-73 years of age, with a median age of 53. Two participants were between 25-40 years old, two were between 41-50 years old, four were between 51-60 years old, and three were between 70-75 years old. Eight participants were female, four were male. All but one of the participants was Caucasian. One participant was African-American. This homogeneity is consistent with the make-up of the area as $93.9 \%$ of WV residents are Caucasian and $3.4 \%$ are African American (WVDHHR, 2010). It should be noted that four of the twelve participants were medical professionals. Three participants were registered nurses and one participant was a physician.

Deceased family members died as a result of various serious illnesses, including esophageal cancer (1), prostate cancer (1), Parkinson's disease (1), ovarian cancer (1), pancreatic cancer (3), breast cancer (1), cancer of unknown origin (1), aortic stenosis (1), and congestive heart failure (1). During interviews, participants described transitional time spans that ranged 
from 3 months (esophageal cancer) to 20 years (aortic stenosis). With the one exception, illnesses persisted for one year or longer. Participants reported that the deceased family member had coexisting health problems, including diabetes, stroke, and renal disease. The causes of death identified by the participants in the sample is representative of the leading causes of death and complicating health problems in West Virginia. Cancer is noted as the leading cause of death in West Virginians between the ages of forty-five to seventy-five years of age followed by heart disease. Heart disease is the leading cause of death in West Virginians seventy-five and older (WVDHHR, 2010). At the time of death, deceased family members ranged in age from 53 years old to 94 years old, with a median age of 70.5 years old.

The research question guiding this study was: How do families retrospectively describe the influence of cultural factors in the transition spanning from the point that a diagnosis with serious illness was made up to the point when the family realized that family members' disease or illness was progressive in nature, that it could be managed but not cured by medical treatment, and would ultimately result in death?

\section{Study Findings}

Findings from this study answer the research question: How do families retrospectively describe the influence of cultural factors in the transition spanning from the point that a diagnosis with serious illness was made up to the point when the family realized that family members' disease or illness was progressive in nature, that it could be managed but not cured by medical treatment, and would ultimately result in death? Findings from this study describe how participants' understanding, behaviors, and decisions at critical points throughout serious illness were influenced by family/kinship, knowledge systems, community/social systems, and faith/religion. Transitions began with the diagnosis of the ill person and spanned to the point 
when EOL was acknowledged. The middle time period was characterized by ebbs and flows in illness and wellness, and included the following critical points in no particular order or frequency: times of wellness, treatment periods, spikes in illness, gradual declines in health, and realization of poor prognosis. Families described multiple critical points during the transition. For example, spikes in illness commonly occurred more than once during the serious illness transition. Furthermore, treatments regularly occurred upon diagnosis periodically during serious illness. For most participants, curative treatments were undergone up until days or weeks before death. It is also important to note that although this study focused on the end point when family members realized the poor prognosis of their relative and acknowledged EOL, the majority of participants described transitions through the death of their family member. Moreover, though most participants $(\mathrm{n}=10)$ could identify a specific point when they realized that their relative would not live despite medical interventions, this point was often very close to the time of death of their family member; in most instances within days to weeks.

Seven themes emerged from the data that describe how the four cultural factors influenced family transitions in illness. Within this report, these themes and sub-themes are organized under four major headings that address the research question. The following themes and sub-themes describe the influence of family/kinship, knowledge systems, community/social systems, and faith/religion on family transitions in serious illness from diagnosis of serious illness to the EOL period.

\section{Family/Kinship}

- Theme 1: Family takes care of family

- Sub-theme: Family circumstances complicated transitions

- Theme 2: We're "going to fight this" 
Knowledge Systems

- Theme 3: "Seeing" and experience were believing

- Theme 4: Families "figured it out"

\section{Community/Social Systems}

- Theme 5: Community eased the family burden

- Theme 6: Health professionals eased and hindered family transitions

\section{Faith/Religion}

- Theme 7: "God was always in the background"

○ Sub-theme: "We prayed and stuff"

○ Sub-theme: Families struggled with their faith as they witnessed suffering Descriptions of the Influence of Kinship on Family Transitions

This section presents how participants described how family and kinship influenced transitions in serious illness. As noted, family and kinship refer to the hierarchy within the family, expectations and beliefs of the family, and family roles and responsibilities (Leininger, 1988). Information gleaned from the interviews revealed that from the point of diagnosis up to the end-of-life (EOL) period, family beliefs and values shaped roles, responsibilities, attitudes about serious illness, and decisions about care in patterned ways. Furthermore, interviews with family members revealed that circumstances occurring within the family during the serious illness complicated transitions in serious illness. Two major themes emerged from the data: Theme one: Family takes care of family and theme two: We're "going to fight this." Within the theme family takes care of family, one sub-theme describes how family circumstances complicated transitions. Themes and sub-themes are presented in the following passages. Participant quotes are included to support findings. 


\section{Theme 1: Family takes care of family}

Family beliefs and values shaped roles and responsibilities in patterned ways throughout transitions. Overwhelmingly $(\mathrm{n}=11)$, participants described the belief that family takes care of family. From the time of diagnosis up to the point when the family member realized that the ill person would die despite medical interventions, family members took care of the ill relative with very little outside help. Outsiders, such as friends and other community members, were rarely mentioned throughout the illness narratives, and when they were mentioned, they played a very discreet role in illness transitions. Furthermore, family took care of family despite other complicated family circumstances that occurred during the serious illness of their relative. There was also evidence that when possible, the seriously ill person tried to take care of their family members as well.

The belief family takes care of family influenced who was involved in family transitions and shaped roles and responsibilities throughout serious illness transitions. Analysis of transcripts revealed that serious illness care involved the entire family, regardless of primacy of relationship to the seriously ill person. Participants described how, throughout transitions, multiple family members were involved in the care of the individual or as a support person for the primary caregiver. Involved family members included members of the nuclear and extended families, and spanned several generations. For example, one participant described siblings, nephews, and a godmother who were involved in the illness of her sister.

I have my oldest sister Jane, and brother, Bill and my nephew. That is my sister who passed, her son, and that is the immediate ... We do have my godmother and she lives in (another state). She is very faith-related, her. So, I talked to her 
and everything, and they did too. And then we had another family member, that's one of our uncles. He was real helpful too, with all of that.

Another participant described the involvement of her step-family in the illness of her father.

I think first to understand, my parents were divorced years ago and my dad remarried. But he had been married to this woman for 35 years. So through my childhood she was my stepmom but we were an integral part. Now, she had four other adult children when she married my dad, and they were close to my dad.

Throughout the illness, family beliefs that family takes care of family shaped roles and responsibilities assumed by family members. Assumed roles included primary care giver, decision maker, and support person. Roles were assumed because of formal family ties, feelings of gratitude and devotion towards the ill person, and/or pragmatism. The role of primary caregiver was described by several participants, and patterns of who assumed this role and why emerged from the data. The primary caregiver role was assumed due to practical reasons or commitment to the ill person. Primary caregivers were usually individuals who resided with or lived in closest proximity to the ill person, had fewer family or outside obligations, and/or chose to make himself or herself available to care for the ill person. One participant remarked on her brother-in-law's (husband to the ill person) availability to serve as the primary caregiver to her ill sister.

Of course he was already disabled and he was home, so I mean it wasn't like he quit work or had any big adjustments, because he had had two hip replacement surgeries and he was already at home.

Similarly, when caregiver and/or decision-maker roles changed throughout illness, it was typically for pragmatic reasons, as described by a participant who lived further away from her ill 
father, "Yes, it became my step-sister over time. She was the one . . because she was there." Other participants described feeling a deep commitment to care for the ill person because of the significance of the ill person in their life, as described by the daughter of a man with cancer. "I made a point of making sure I was there every single day of my life. They raised me the majority of my life, and I couldn’t think of anything else I wanted to do.”

In this study, four of the twelve participants were males. Two males acted as primary caregiver to the seriously ill person. One male described discomfort bathing his mother, and expressed gratitude that his wife provided this care instead, “...like helping her [my mother] even have bathes or... Without her [my wife], I mean, I don't know what I would have done." This finding may speak to cultural norms and ideas related to the propriety of males providing intimate physical care to females.

Participants described patterns in assumption of decision making roles. Six participants stated that the decisions were made by the seriously ill person. These individuals were described as being independent and knowing what they wanted, as depicted by one participant's description of her seriously ill mother-in-law; "She's a strong lady so she pretty much made her own decisions." Another participant's statement described her mother as her own decision maker - even making decisions that conflicted with the actions taken by the family and recommended by the physician. "I had an appointment with him for her to go and she made me cancel it. She said that she didn't want to go. She didn't want to start . . and that was her choice."

Four participants described decision making roles as being shared by two or three individuals. In these families the ill person was always included in decision-making and often retained the final say. Dyads typically included the ill person and his/her spouse, as described by 
the husband of a woman who had cancer. "My wife and I probably made the decisions as to what medical care she would or would not receive." In addition to the ill person and his/her spouse, trios involved an adult child. Family members who did not have a final say in actual decisions were often involved in the decision-making process by providing input, offering advice, or supporting decisions made by the primary decision-makers.

Family members who did not act as primary caregivers or decision makers remained involved and connected to the family by playing more supportive roles throughout the illness. Reasons cited for not being the primary caregiver were pragmatic, and included limited availability due to work and/or family obligations and/or physical proximity to the ill person. Several participants simply stated "we weren’t as involved" without providing additional clarification for why they were not as involved. A statement from one participant depicts the numerous practical reasons that some other family members could or did not act as primary caregiver.

They have a son that lives in Arizona and he was just not able to be here. I mean, he was a support for me over the phone, but he just couldn't be here. They have another daughter who lived in Georgia at the time and she would come in or backand-forth, but she had a life and she wasn't really interested, per se. And then they have another daughter that lives locally, but she worked and she couldn't always be there.

Family members acting in supportive roles provided counsel and emotional support to primary caregivers and other family members through face-to-face and phone conversations, as described by the son who served as a caregiver to his seriously ill mother. "Of course we [my brother and I] talked. You have to try to prepare for stuff, you know?" They would provide 
respite to the primary caregiver by occasionally taking the ill person to appointments, sitting with them, or staying with them in the hospital, as described below by the husband of a lady who was diagnosed with pancreatic cancer.

They [our children] were just always in and out - Anything we can do? What can we do? They were willing to take us anywhere. If I didn't feel like driving or what have you. But just total support.

Analysis of data also revealed that the ill person believed that family takes care of family. Participants described behaviors and actions of the ill person in order to spare other family members emotional or physical burden. One participant described how her uncle withheld information from his children to spare them worry and distress.

I don't think he wanted to worry his kids..., and I think he didn't want to scare them into wanting to come back and spend every minute with him, because they were finally getting on their feet a little bit.

Another participant described how her father decided to enter hospice care to decrease the burden on his wife.

I think she called and I think he was a little resistant at first, but when they [hospice] came and they kind of laid it out, I think he realized that it was a lot on her. It was wearing her out.

Participant beliefs that family takes care of family were evident even when other circumstances collided with the serious illness their relative. A description of family circumstances that complicated transitions follows.

Sub-theme: Family circumstances complicated transitions. Large, diverse family structures often meant that in addition to the illness of their family member, families were 
experiencing other circumstances which complicated transitions. Participants described difficult circumstances or situations within their family that converged with the illness of their family member and complicated matters $(n=5)$. "Other stuff" included loss of another and/or simultaneous illness of another, and disagreements or discord within the family. Deaths of other family members were sometimes linked to the decline in health of the ill individual. "Then, my grandfather passed away, and then after my grandfather passed away it seemed like she [grandmother] got worse and worse." Deaths and illnesses of other family members also increased physical and emotional burdens on families as they had to divert attention to others' needs as well. One participant described dealing with the same diagnosis of his daughter's father-in-law while simultaneously dealing with his friend's diagnosis of pancreatic cancer. Ironically my daughter's husband's father is [was] also diagnosed with pancreatic cancer, and the diagnosis came within [about] the same time. So dealing with my son-in-law's father's issues and dealing with my friend's issues, this cancer business, just was a compounding travesty, really.

Another participant described experiencing the traumatic accident of her brother while simultaneously experiencing the illness of her sister.

Yes, and in between this time, right before she [sister] passed away, my other brother was coming from work and down (the interstate) and had a really bad car accident and he was in (the hospital) for two or three months up there, recovering from that accident, because he had extensive, trauma to his stomach area and broken bones. So, we were going through that too, at the same time. So, I mean there was a lot of stuff to deal with all at once. 
Discord within the families was also described by four participants. The described discord was a result of jealousy and broken family relationships, as well as other complicating matters, and added another layer of complexity to an already complex situation. One participant described tension with her mother throughout the illness of her father that resulted from their family separation and subsequent divorce.

My mom was supportive, but she ... I was more guarded with her, because they [divorced parents] had a very bad relationship. It was always bad. It was always horrible. It was always just so . . I I think my mom wanted me to tell her more, but I just kind of felt like I didn't want to take a chance, that some of the old stuff might come back in. You know? So I was just was like - Oh, whatever. Yeah. Another participant described jealously and tension within the family.

There was some jealousy in the family. You know, I think the others were jealous that I was there, but it wasn't because they weren't allowed to be there. It was because they chose not to be there. You know, I mean there was some . . so there was a lot of tension at times and it was rough, and to this day there is still some tension.

These findings revealed that family members did not experience the illness of their relative in isolation, many times family members were experiencing other critical life events as well. Simultaneous illnesses of other family members, traumatic accidents, deaths, and family discord all increased stress on family members throughout the illness trajectory. These other circumstances complicated transitions by not allowing certain family members to be as attentive to the ill person as they otherwise could have, not being able to involve other family members as they would like, and not being able to process information as they should. Family members had 
to divert attention away from the ill person to tend to other circumstances. In spite of this, family members' believed that they should be involved in the care and support of the ill person, and tried to remain as involved as possible throughout serious illness transitions.

Though family structures varied among participants, a constant finding was that participants' believed that family takes care of family and were, therefore, involved in serious illness transitions. Family members assumed a variety of roles throughout transitions in order to take care of family. Regardless of practical limitations, it was evident from family members' descriptions that the serious illness was a family affair and kin takes care of kin. This overarching belief that family takes care of family presided in spite of other circumstances that occurred simultaneously with the serious illness of family members and complicated family transitions in illness.

\section{Theme 2: We're "going to fight this."}

Family beliefs that we're "going to fight this" shaped attitudes about serious illness, what types of care were sought and when they were sought, treatment goals, and decision making. Five participants described beliefs of "fighting" and "not giving up" when it came to making choices about care. Families described choosing treatments in order to keep fighting the disease. This belief was most prevalent in the early stages of illness, but for some participants persisted through the very end stages of illness, as exemplified by the following quote. "I always vowed that I would give $110 \%$ in the fight, and I wouldn't give up until it was over, and I told him that from the get-go, that I would never stop fighting for him." Three participants described rejecting hospice because they weren't ready to give up the fight, as stated by one participant, "We fought Hospice.... we weren't ready to give her up, so they came out one time and we said - No, we're not going to just accept Hospice, that she's going to die. We're going to fight this tooth-and-toe 
nail.” Though not explicitly stated, other participants exhibited this belief through their described behaviors and actions throughout illness. For example, one participant described seeking rehabilitative care for her mother-in-law who was suffering from Parkinson's disease so that she would be well enough to go home and independently care for herself.

The age of the ill person sometimes shaped family beliefs about fighting and not giving up. Three participants mentioned attitudes towards age and illness as a factor in making decisions to pursue more aggressive treatment options or to accept poor prognoses. One participant described her belief that older adults do not readily bounce back from illnesses, therefore conceding the fight. "You don't come back from that, not real fast, and at 62 years old, not real well. Now, if she had been 22 it would have been different." Conversely, another participant described difficulty coming to terms with the diagnosis not surrendering because of the young age of her uncle. "He hadn't come to terms with it yet. None of us had, because he was 54. We didn't see it coming."

The mindset of the ill person also influenced family members' beliefs to keep fighting the illness. In the earlier stages of illness, the ill person was more likely to want to fight the illness as well, further prompting the family to continue the fight. "So my dad kept asking me like Well son, what do I need to do? Stuff like this. And - What can we do to beat this? His mindset was that he was going to beat this." However, as time went on and the ill person became tired and weaker, he/she would sometimes lose the desire to fight or not want to burden family members with care needs.

Family beliefs that we're "going to fight this" influenced decisions about care throughout illness transitions, from diagnosis to the EOL period. This belief led families to choose more aggressive treatment measures and forego or delay hospice care services. This belief was most 
influential in the early stages of illness, however, for some participants, persisted to the end stages of illness.

\section{Descriptions of the Influence of Knowledge Systems on Family Transitions}

Knowledge systems refer to what was known about the illness, what was expected because of that knowledge, how families came to know or realize the progressive nature of the illness, and where families turned for answers and/or advice during the illness (Leininger, 1988). Throughout serious illness transitions, knowledge systems influenced family understanding of illness, care seeking behaviors, decisions about care, and realization of prognosis. Two overarching themes with two sub-themes emerged from the data that described the influence of knowledge systems on family transitions from diagnosis to the EOL period. Theme 3: "Seeing" and experience was believing. Subthemes visible cues and previous experience further elucidate this theme. Theme 4: Families "figured it out", describes how families "figured it out" by talking about it and educating themselves, primarily when they did not have information they needed or had conflicting information. A complete description of the themes and categories supported by participant quotes is in the following passages.

\section{Theme 3: "Seeing" and experience were believing}

Participants described "seeing" or not "seeing" changes in their ill relative that led them to believe that the ill person was well or ill and prompted family members to seek or not seek out care. "Seeing" also led families to realize the poor prognosis of their relatives at times.

Additionally, family members described how knowledge derived from previous experiences with illness as well as formal medical or nursing education shaped their understanding of the illness and decisions about care. This was sometimes in conflict with what they were told by health care professionals throughout the illness. 
Overwhelmingly $(\mathrm{n}=11)$, participants described knowing that the ill individual was "OK" or not "OK" throughout the course of illness because of visible clues. Visible changes were often observed by family members before they were recognized by health care providers. Visible changes influenced how family members perceived illness, and patient and family decisions to seek or not seek care. After the initial diagnosis and treatment of the ill individual, participants described periods when the ill person was "OK", which were typically denoted by no apparent signs and symptoms of illness and the ill person being able to carry on daily activities normally. During these times, participants and their family members typically did not seek medical care or advice, and often described thinking that everything was "OK". One participant, a husband to an ill person with pancreatic cancer, described his wife as being "OK" after surgery and treatment for cancer.

But yeah, we just went back ... she went back to help me in the office and taking care of the books and things like that, from October the 5th until the 1st of March of 2012. We thought everything was fine and she didn't have any problems. I mean, she just carried on normal life and worked in the house and just the same old Maureen. You know?

Participants also described having hope because of visible, noticeable improvement of their seriously ill family member. This was sometimes in spite of the participant's knowledge of the severity of the illness. One participant, who was also a nurse, described the hope that she had for her uncle because of changes that she could see in him.

Finally, when they started getting him, at (Treatment Center) and getting him on good pain control and things like that, he quit doing that. And that was nice to 
see, and that kind of gave us hope that he was getting better, but that was kind of hard.

Family members who had a medical background or those that chose to become more knowledgeable about medical treatments and terminology, used diagnostic tests and lab results as ways they could "see" if the ill person was improving or not. "You know, he went home. He started doing better. He started eating pretty good and his CA-19 dropped down to like 90, almost immediately." One participant described how seeing the results of a diagnostic test led to the realization that her father would not recover, "But when we got those results of that it was a different . . . it was a game-[changer] . . I mean, it changed everything. You know?”

Visible changes also alerted the family and ill person that they were no longer "OK" and prompted the patient and family to seek medical care. One participant noticed a change in the color of his father that led them to seek care. "And so the end of April, the 1st of May, he starts to turn yellow again." Participants also realized that something was wrong because of other telltale signs that something wasn't right.

At the point we realized something was seriously wrong was that he could not lay down at all. Like, he would lay on the couch and he would jump back up. Like he couldn't stand to lay in any position.

Seeing changes also led to the realization that the ill person would not recover from their illness and would eventually die. "I could tell the brain damage had gotten really bad by that time...But I knew she was not going to come back and be normal and I just wondered how long."

Throughout illness transitions, from diagnosis to the EOL period, visible clues shaped family understandings of illness, care seeking behaviors and realization of prognosis. Families believe that their relative was "better" when they could "see" them carrying on as normal, and 
therefore did not seek out care. Conversely, when they "saw" physical and functional changes in their relative, they believed that something was wrong and were more likely to seek out care. Finally, visible declines in health sometimes helped the family to realize that their relative would not recover from the illness. In addition to visible clues, families also relied on previous experiences and education to understand the illness and make decisions about care. These findings are further explained in the following passages.

Family members' previous experience with illness and/or formal medical and nursing education shaped what they understood about the illness and decisions about care. Seven participants described what they knew about diagnoses, treatments, and prognoses because of past experiences. Past experiences typically included incidents with other ill family members or other ill persons. Knowledge gained from past experiences influenced care choices as well as beliefs about recovery from illness. One participant described deciding to forego chemotherapy because of what he knew as a result of his brother-in-law's experience with chemotherapy.

We had talked about this, that if they find cancer that we don't even want chemotherapy because she had lost her brother to cancer - not cancer but from the chemotherapy therapy. He had had it two or three times and it was the chemotherapy that wound up killing him.

Yet another participant described how her work with persons with brain damage influenced her understanding of her sister's prognosis. "I worked out at the hospital and stuff, and I have been around people that ... and you know they don't come out of it."

As noted, four of the participants in the study were also health professionals. Three participants were registered nurses while one participant was a physician. Upon diagnosis, these participants described knowing something about the disease and what to expect because of 
medical or nursing education. One physician remarked on his initial understanding of the diagnosis because of his formal medical knowledge. "I was not unaware of pancreatic cancer epidemiology, the outcome, the chemo-therapeutic agents that are available and the eventual fact that this is basically a death sentence at Stage IV pancreatic cancer." Another participant, who was also a professor of nursing, described knowing about the diagnosis because of formal education. "I knew about cancer, because I teach oncology, here."

Knowledge gained from formal education influenced the role that individuals played throughout the illness as these family members were turned to for information or advice throughout the illness and would answer questions about diagnoses and care $(n=3)$. Two participants who were nurses described family members turning to them for information and advice throughout the illness. "He wanted to know, you know, what this meant and he would read off test results to me over the phone. And my sister is a nurse too, so he would kind of consult with both of us." Other times, participants described giving advice or making suggestions to the ill person or the primary caregiver because of their formal medical knowledge. I said - After surgery, I don't care what they tell you, I said you need to be up and moving. I said - I don't want you laying in that bed. I said - Whenever you wake up, you've got to get moving.

These participants also described the difficulty that they had balancing the roles of family member and information broker, as described by the son of one ill person, who was also a nurse. "There were times like - Okay, I need to ask somebody else. Am I seeing this the same way, or is it just because I'm too close to the situation?" Furthermore, these participants noted that in spite of their formal education, they were not really prepared for this experience with their family member. 
I didn't realize how hard . . . Because you know, I've worked in ICU for many years, and I've worked oncology for a lot of my career, but when you are actually doing this hands on, hands on stuff with your own family it's tough.

Perhaps one participant, the daughter-in-law of a woman with serious illness and a nurse, summarized this best when she stated, "I mean, until you experience it, you never know what to even expect. “

At times, previous knowledge was challenged as family members came to learn new or different information at critical points in the illness trajectory. Regardless of formal medical or nursing education, family members had to reconsider approaches to care and decisions about care because of this presupposed knowledge. For example, one participant described what she knew about cancer treatment decisions, but then came to find out differently.

That's what I thought, because I always thought you had to have chemo. I didn't think it was like the patient's decision. I guess they gave you the option and they tell you what all the options are, and then you have to make the decision.

Another participant, who was also a nurse, described learning something new throughout the course of her uncle's illness that challenged her presumed understanding of acceptable treatments for her uncle.

I knew his white blood cell count would be low, so when flu season came around I was like - We need to make sure that he gets a flu shot. At (the treatment center) I actually learned something new. They said the flu shot would basically be a waste of time, because as soon as it got in him the chemo would kill it. I didn't know that. I was like - Huh, I learned something new. 
Two participants described coming in and out of hospice because they initially thought that hospice could be done in conjunction with curative measures, as illustrated by one participant's remark, “And then we took him out, because we didn't realize that you couldn't be on chemo and be in Hospice." Obtaining knowledge that was contradictory to original understandings of illness and treatments often influenced care choices. Participants had to rethink original ideas and understandings because of new knowledge.

Knowledge from visible clues, experiences and education influenced understanding of illness and decisions about care. Visible clues alerted family members that something was wrong and often times led them to seek out care. Conversely, visible clues also led family members to believe that their relative was "OK", and therefore not seek out care. Experiential and educationally derived knowledge shaped interactions with health professionals, choices of care providers, choices of treatment, and realization of prognosis. Furthermore, previous knowledge was sometimes challenged as family members progressed through transitions and learned new information that was contradictory to previous beliefs, causing family members to re-evaluate the situation. In addition to visible clues, experience, and education, family members described various ways that they "figured it out" within the confines of the family throughout illness transitions. Theme 4: Families "figured it out" is presented in the following passage.

\section{Theme 4: Families "figured it out"}

Participants in this study commonly described "figuring it out" when they had limited knowledge or understanding of the illness or a specific circumstance that occurred throughout the illness. From diagnosis to the EOL period, families "figured it out" by talking about it and educating themselves. Participants described patterns of family communication throughout the illness that influenced understanding of treatments, decisions about care, and realization of 
prognosis $(\mathrm{n}=9)$. Communication patterns ranged from withholding information to full disclosure of information, and often included multiple family members. Some families reported that their ill family member did not always share information about their illness, prognosis, or treatments with them $(n=4)$. This occurred primarily towards the beginning of the illness, particularly when the prognosis was poor. One participant described how her father and step-mother withheld information from her and her step-siblings and how that hindered her ability to come to terms with the illness and what to expect.

But, no one was with him when the oncologist came and told him that it had spread to the liver. So, we didn't have a lot of information about . . . you know what I mean? ... how much of it had spread.

Two participants stated that their families "didn't really talk about stuff like that," referring to the illness and prognosis of their family member. "They never did. My sister and them [siblings] never did talk about any of that." Family members with incomplete information did not always realize the seriousness of the prognosis.

The majority of participants described discussions with their ill family member and other involved family members such as children, siblings, nieces and nephews throughout the illness $(n=9)$. Conversations typically arose when families noticed or learned something new, didn't understand symptoms or treatments that they witnessed or were undergoing, or had to make a decision. During these discussions families shared information, came to understand circumstances, and made decisions. Several participants described making care decisions after talking about it with the family. "We talk to her about doing dialysis, and she didn't want to do it. She wouldn't have made it anyway, not with the heart." Other participants described relaying decisions to family members during family talks. 
But we did discuss it with each of our children, and we would have several family conferences and let the kids know what we had decided, and of course they would ask questions but they would never question to the point of trying to change our minds about things they knew that had to be our decision.

One participant described how she and her siblings came to realize the poor prognosis of their father's illness after talking about it.

So we just communicated together as siblings and it was at that point that I realized that we probably didn't have as much time as we thought we had. It had probably much ... had progressed much farther along than any of us were kind of given the impression to believe.

Talking about it helped families to convert knowledge into realization. Participants also described figuring it out by educating themselves. Family members described ways that they educated themselves on diseases, diagnostic tests, and treatments and figured out what was going on with their ill family member at multiple points throughout the illness trajectory. Selfeducation involved individually seeking information from Internet sites and asking questions to health professionals. The knowledge gained from this self-education influenced how the family made sense of and understood the illness. Five participants described educating themselves by searching for information on the Internet. Looking up information on Internet sites was most common in the beginning stages of illness, when the diagnosis was first made, as well as when new tests or treatments were introduced. The following quote depicts families' use of the internet to educate themselves in order to gain a greater understanding of the illness and treatments and be better prepared to manage the illness of their family member. "We actually go on the Internet, 
like everybody does, and read about it, and just, you know, kind of try to educate ourselves to know what to do if we were in that situation on our own, with her."

Asking questions of health professionals took place at the beginning and throughout the illness, particularly when ill persons and their family members noticed that something wasn't right or received new information and/or diagnostic results. One participant described how she asked questions when presented with new results; “And, when there was any type of result I would ask the questions." Another participant described how her family asked questions in order to educate themselves on the illness and treatments. "No, we really had to educate ourselves on the matter, and then just ask questions to the doctor and the nurses."

Family members were resourceful and "figured it out" through a variety of mechanisms. Internal family conversations were important to help families understand the illness and realize the prognosis of their relative. Self-education included seeking information from the Internet and asking health professionals. By "figuring it out", families were able to better understand the illness, make decisions about care, and realize the poor prognosis of their relative.

\section{Descriptions of the influence of community and social systems on family transitions}

This section presents two themes and two sub-themes that describe the influence of community and social systems on transitions in illness, from diagnosis to the EOL period. Community and social systems include the relationships and interactions with persons outside of the family, such as friends, neighbors, support organizations, employment organizations, health care organizations, and health care providers. Theme Five: Community eased the family burden describes how friends and neighbors eased the burden by filling in for family during times of duress. Theme Six: Health professionals both eased and hindered family transitions describes how health professionals were helpful to families throughout transitions and, conversely, how 
they were not helpful. A full description of themes along with participant quotes that support findings is presented in the following sections.

\section{Theme 5: Community eased the family burden}

From diagnosis to EOL, friends and neighbors eased the family burden by filling in during times of duress. Friends, neighbors, and church communities were often described as extensions of the family and provided physical and/or emotional support during the illness, specifically during times of duress or when the family was especially busy with care of the ill person $(n=8)$. Helpful acts provided by the community included meal preparation, respite care, and help with transportation and child care responsibilities. These acts relieved some of the physical day-to-day burdens from family members so that they could focus more on the care and needs of the ill person. One common act performed by friends and neighbors was the preparation of meals. Multiple participants described community members preparing meals for them during times of duress, as described in the following quote: "They tried to bring some meals and different things like that. So, they were a support, but it was helpful.” In addition to preparing meals, one participant described how neighbors helped care for her children so that she had one less worry while her father was sick.

They were wonderful. I mean all of my neighbors were, because they had to help me take care, you know, get kids off the bus and take them to different activities and I really didn't have to worry about a lot.

Work friends were described as accommodating schedules so that families could be involved in the care of the ill person. 
My husband's work community, they were very understanding that he was going to have to take some time off and he was going to have to be home earlier to help get kids shuttled where they needed to be.

Other participants described offers from co-workers to provide respite care. This was most common with participants who were health professionals.

I had some of my co-workers say - Hey, if you need somebody to come over and sit with him while you guys get out, you guys just tell us what you want and we will be more than glad to help.

Friends and neighbors were important to transitions because they acted as extensions of the family and eased the care burden. Though health professionals were not described as proxies to the family, they too influenced transitions in illness. Unlike friends and neighbors, health professionals both eased and hindered family transitions in serious illness, from diagnosis to the EOL phase. These findings are described in the following section, under the theme: Health professionals both eased and hindered family transitions.

\section{Theme 6: Health professionals both eased and hindered family transitions.}

Participants described the influence of health professionals throughout transitions in serious illness, from the time of diagnosis to the EOL period. Health professionals had both positive and negative influences on family transitions in serious illness. Helpful behaviors included caring for and supporting families in transitions and helping families to "figure things out." Conversely, health professionals hindered family transitions when they weren't attentive to family needs, did not communicate with them, or didn't know what to do. Positive and negative behaviors of health professionals influenced the understanding that families had about illness and decisions they made about care. 
Behaviors and acts of health professionals throughout the illness facilitated transitions by allowing family members to feel cared about and supported, and helped families "figure things out." Multiple participants described helpful acts of health professionals $(n=9)$. A variety of health professionals were mentioned throughout the interviews, and included physicians, nurses, physical therapists, social workers and nurses' aides. Supportive behaviors demonstrated a sense of caring about the patient and family.

In addition to generally supportive behaviors, participants also described health professionals that helped them "figure out" what was going on throughout the illness transition. Honest, forthright, individualized communications were valued by participants, and allowed families to better understand what to expect throughout the illness. Participants appreciated health professionals who took the time to listen to their needs and make sure that information was well understood. One participant's remarks reflect the appreciation for caring supportive behaviors as well as valued communication practices.

He was very nice. He was very caring and he was very sincere. That is what I think I hooked onto most, was how sincere he was. He cared. He was the doctor that it didn't matter if it was a 5-minute appointment or 30-minute appointment. He wanted to make sure that we understood and he wanted to make sure we was okay with the information that he had.

Another participant described the helpful acts of a social worker in figuring things out when trying to determine treatment options for her mother-in-law.

I think the social worker at the nursing home was helpful. She really helped us to try to figure out, she said - If this rehab doesn't work how could we look at, what did we want to do? Did we want to do long-term care? 
Nurses who provided answers and information to family were also described as helpful. "I always felt like if I had a question I could contact the nurses and I felt like we were okay, you know, like they would help us answer our question.”

Families credited health professionals with "telling" them vital information throughout the illness, particularly in the end stages $(n=5)$. Participants described coming to realize that the ill person would not get better because of communication with health professionals. This particular act of health professionals was described both positively and negatively by participants. Positive descriptions alluded to honest, caring, timely provision of information. One participant, the son of an individual with cancer, described how his father's physician discussed end of life care options.

The doctor, he come out and - Edward, I want to talk to you out here. He says Edward, I honestly believe the cancer is back. He said - I don't think there's really anything we can do. They said - We can send you to (hospital) if you want, or if you want to have quality time, go home with your family.

Families appreciated health professionals who demonstrated caring behaviors and shared information in a timely, respectful manner. These behaviors eased family transitions in illness by helping family members understand the situation. Conversely, families described how health professionals could also make transitions harder.

Participants described behaviors and actions of health professionals that were not helpful throughout the illness as well. Such behaviors and actions included not being attentive to family needs, not communicating with families, or not knowing what to do. These behaviors and actions hindered transitions in illness by diminishing trust between families and care providers, delaying treatments, and impeding family comprehension of illness, treatments, and prognoses. 
At multiple points throughout transitions in illness, participants described not feeling cared about by physicians and care providers. This lack of caring shaped family opinions about care providers and care institutions and influenced how families interacted with care providers. Sometimes, participants describe basing decisions about care on behaviors of health professionals. One participant remarked about the lack of concern they got from one facility upon initial diagnosis with serious illness, "So, we went to (this hospital) and they just kind of blew him off. It was just not a good experience." This participant further went on to describe going elsewhere for care because of this experience

Lack of faith in health professionals because of their non-caring behaviors was described by several family members, who interestingly, were also in the health professions. One participant, a nurse, described her frustration with care providers who did not take the time to determine or accommodate her mother-in-law's individual needs.

The woman couldn't even walk. You know? And, they didn't know who I was, from Adam, whether I could help or whether I knew how to do a clean-catch urine or anything. To me that was so frustrating. I thought - If I wasn't there as a support for her, I don't think they would have really been able to . . . I don't really know what would've happened.

Another participant, also a nurse, described her disappointment with health professionals because of multiple incidents that occurred throughout the illness. She described having to interfere in situations to ensure that her uncle received appropriate care.

There were other things that happened that really bothered me, that if I wasn't in nursing and knew what was going on, there was things that I'm going - What 
would have happened if somebody wouldn't have stepped in and said - Hey, we need to back the truck up here.

Participant actions, behaviors, understanding of illness, and decisions about care were influenced by the behaviors of healthcare professionals. Furthermore, family members became distrustful of health professionals who did not display caring, concerned behaviors towards patients and families.

Poor communication with health professionals hindered families understanding of the illness and treatments and led to frustration with health professionals. Poor communication practices were described by six participants, and included withholding information, poor timing of communication, being told incorrect information, and not taking adequate time or showing concern when communicating with families. One participant described a physician's inability to provide clear information and how not being forthright led to care choices that they otherwise would not have made.

If the doctor would have really made it clear - I don't think we have more than three months ... I would have been there and I could have put things in place and that would have alleviated some stress on my stepmom and my dad.

Another participant described his frustration and disappointment with being told incorrect information by physicians. The information provided by physicians led to care choices that had he been told otherwise, might not have made.

I say that doctors should not be allowed to do this, but they come out all beaming after operating on her with a laparoscopy for six hours and they just knew that they got it all and stuff, and you know, they bring your hopes way up, and almost never do they get it all. So I said that I wish there was a law banned doctors from 
even saying that, because if that hadn't been the case we probably would have followed up with chemotherapy and not radiation therapy.

Poor timing of information and omission of information by health professionals were described by participants. Two participants described being referred to hospice care before they had time to come to terms with the illness. Both families came out of hospice, and pursued treatment for at least one year before coming to the realization that their family member would not recover from their illness.

Honestly at that point we didn't know. We had no clue. We was still trying to figure everything out and everybody said - Well, let's just bring in Hospice. And once they come in and they started explaining things to us, and I'm like - Wait a minute! We're not stopping ... I mean, we're not going to not do treatment.

Late delivery and omission of information was also described by participants and hindered family transitions in illness by presenting barriers to family members understanding of illness. Participants described not being told critical information, not being told about all treatment options, such as hospice, or being told information much later than the health professional knew about it. One participant described her frustration with not being told about critical diagnostic results until three months after the test had been conducted.

Well, when I got to looking at these medical records, this was from a PET scan that was done in April, and we hadn't learned that information until two months later or three months later, and I think that was at the point I was ready just to choke him.

As described, non-caring behaviors and poor communication by health professionals complicated family transitions in illness. Participants struggled to understand what was 
happening at specific points throughout illness transitions because of health professionals' actions and behaviors. Furthermore, health professionals sometimes hindered the participants' ability to make decisions, especially in the later stages of illness. Yet, there were also times when families described how health professionals not knowing what to do led to trying something else throughout transitions.

When physicians were unsure of what was going on, couldn't make a diagnosis, or felt the problem was beyond their scope of practice, they referred the patient and family elsewhere or "tried" another test, procedure, or course of treatment to figure out what was going on or to try to alleviate symptoms $(n=7)$. One participant described "trying" a rehabilitation center to improve her sister's mobility when nothing else worked. "After I left they decided to move her to a rehabilitative center to see if they could get her mobile on her legs so that she can get home. So they did that." Medications were also "tried" to help alleviate symptoms when the cause of symptoms was unknown. "They tried him on different medicines and you know, he would be okay, but then he was hesitant to eat, and lost massive weight." Several participants described being referred to specialists or other health care providers when the current physician no longer had answers.

Dr. Barnard he said - Well, you really look worse. He said - I don't know what's going on. He said - We can't find anything different. But he said - I have a friend who works at (a specialty hospital), so would you consider going there?

The uncertainty of physicians and their reluctance to acknowledge the limits of medicine led ill persons and their families to try another treatment, procedure, physician, or care facility to get answers or care. 
Health professionals complicated transitions by their behaviors and actions.

Inattentiveness to family needs, poor communication practices, and uncertainty influenced family members' understanding of illness, decisions about care, and realization of prognosis.

Many times, the behaviors of health professionals caused family members to not fully understand the illness or treatments, avoid health care services, attempt unnecessary treatments, or have delayed realization of poor prognosis.

\section{Descriptions of the influence of Faith/Religion on Family Transitions}

The belief in a higher deity and the performance of religious customs and rituals influenced transitions in illness. The role that faith and religion played in illness transitions varied among participants, yet the belief that "God was always in the background" was consistently described by participants in the study. Two sub-themes further elaborate on findings: "we prayed and stuff" and families struggled with their faith as they witnessed suffering. The following passages provide a full description of theme 7 and sub-themes, as well as quotes from participants to support findings.

\section{Theme 7: "God was always in the background"}

From diagnosis to the EOL period, "God was always in the background". The majority of participants described religion, particularly Christianity, as playing a role in illness transitions $(n=9)$. Religious rituals, such as praying, occurred throughout illness transitions, from diagnosis to the EOL period. These rituals are further described under the sub-theme "we prayed and stuff." Faith, which is more of a conviction, belief, or trust in a higher deity, contributed to internal struggles throughout the illness as they witnessed suffering or their family member. These findings are discussed under the sub-theme families struggled with their faith as they witnessed suffering. 
Sub-theme: "We prayed and stuff." The majority of participants stated that they prayed upon learning of the diagnosis and throughout the illness $(n=9)$. Prayer was described as both a ritualistic practice and a faith-based practice by participants. In most instances, prayer was an individual practice. Participants described prayer as a ritual that affirmed their Christian beliefs and give them hope.

I consider myself a Christian and I believe in God. I'm not a churchgoer, but you know, I believe in prayer and that kind of stuff. You know, I prayed a lot and stuff like that.

For others, prayer was a testament to their belief in God's healing powers. One participant described partnering with his church community and using prayer for its healing powers throughout his wife's illness.

We had different people coming into our home and praying for her and laying hands on her and we really felt this was almost like a test case for our prayer group, just get her well.

Though prayer was described as a positive influence in family transitions in illness, participants also described faith as contributing to internal struggles as they witnessed suffering of their relatives throughout the illness trajectory.

Sub-theme: Families struggled with their faith as they witnessed suffering. Participants described struggling with their faith as they tried to come to terms with the illness and the suffering that it caused their family member $(n=4)$. Participants struggled to understand how God could allow "good people" to suffer. One participant described a similar struggle when watching his wife in agony from her cancer. 
I can't fathom all the agony she went through, and why she had to go through that. You know? That's the hardest part to understand. That's even harder than seeing the suffering they're going through, the pain and stuff.

Two family members who were in health professions described the struggle between faith and the scientific knowledge that they possessed. Their knowledge of the disease, treatments, and prognoses sometimes made it difficult to fully rely on faith. One participant, who was also a physician, discussed the struggle that he had balancing scientific knowledge with beliefs in the power of a super-natural entity.

God and all that was always in the background, always thought about, always questioned, and always put into perspective. As scientists, that we're supposed to be, looking at facts, it is very difficult for a lot of us to come to grips with the idea of a supernatural entity beyond, and what we're able to see, feel and touch.

Though participants believed in a God and expressed faith in God, their faith also caused internal struggles as they tried to come to terms with and understand why their family member was suffering.

Faith and religion provided participants with rituals that allowed them to find comfort at critical points throughout transitions in serious illness. Furthermore, for some participants, prayer offered hope for cure. Participants described praying individually and with others to help their seriously ill relative. However, faith also caused internal struggles for participants as they tried to make sense of the suffering that their relative experienced throughout the serious illness.

\section{Summary}

Chapter four discussed the findings of this study of families' retrospective descriptions of the influence of four cultural factors on transitions in serious illness. Specifically under 
investigation was the time period spanning from when families first learned of the illness diagnosis up to the point when the family realized that the family members' disease or illness was progressive nature, that it could be managed but not cured by medical treatment, and would ultimately result in death. Cultural factors of interest included kinship/family, knowledge systems, community/social systems, and faith/religion. The researcher identified seven themes that answered the research question. The study findings revealed family/kinship, knowledge systems, community/social systems, and faith/religion influenced transitions in serious illness from diagnosis to the EOL period. Critical points and times included diagnosis of illness, treatments, periods of wellness, spikes in illness, gradual declines in health, and realization of imminent death. All factors were described as both positively and negatively influencing family actions, behaviors, understanding, and decisions throughout transitions in illness. Throughout the illness, these factors were described as influencing family roles and responsibilities, understanding of illness and treatments, interactions with health professionals, awareness of situations, and decision-making. Perhaps the most notable finding from this research is that serious illness really is a family affair. Though other factors influenced transitions in illness, family and the ill person were always at center. Family and kinship were constant influences throughout transitions in serious illness. Other factors played a more peripheral role and were included at specific points in time throughout the illness as needed. Thematic findings from this study and their implications will be further discussed in chapter five. 


\section{Chapter 5}

\section{Discussion of Findings}

The purpose of this qualitative study was to capture families' retrospective descriptions of the influence of cultural factors in serious illness transitions. The study was guided by the research question: How do families retrospectively describe the influence of cultural factors in the transition spanning from the point that a diagnosis was made up to the point when the family realized that the family members' disease or illness was progressive in nature, that it could be managed but not cured by medical treatment, and would ultimately result in death? Specific cultural factors of interest were family/kinship, knowledge systems, community/social systems, and religion/faith. The concepts of family transitions and culture framed data collection and conventional content analysis methods were used for data analysis and reporting of findings.

As noted in the previous chapter, the following themes describe the influence of family/kinship, knowledge systems, community/social systems, and faith/religion on family transitions in serious illness: (1) family takes care of family; (2) we're "going to fight this;" (3) "seeing" and experience were believing; (4) families "figured it out;" (5) community eased the family burden; (6) health professionals both eased and hindered family transitions; and (7) "God was always in the background." The purpose of this chapter is to present a thematic summary of the findings in relation to concepts of transitions in serious illness and culture; more specifically the cultural factors family/kinship, knowledge systems, community/social systems, and faith/religion. A summary of findings is presented. The findings are then discussed within the context of relevant theories and research. Implications for nursing practice, education, and research are also presented. 


\section{Summary of Findings}

Findings from this study describe the influence of family/kinship, knowledge systems, community/social systems, and faith/religion on family transitions in serious illness from the time of diagnosis up to the end-of-life (EOL) period. Family members described transitions that began when they first learned of the diagnosis of serious illness and spanned up to the realization of poor prognosis, which was typically within days or weeks of death and denoted by acknowledgement of poor prognosis and/or cessation of curative measures. Within this time span, family members described ebbs and flows, including times of wellness, when the ill relative was able to carry on normal day to day activities and had no apparent signs of illness; periods of treatment, which occurred off and on at various times throughout the illness; spikes in illness, where the seriously ill person experienced symptoms that required intervention; and gradual declines in health, which typically followed spikes in illness or led up to the realization of poor prognosis. Family members described how the four cultural factors influenced their understanding, behaviors, and decisions about illness, treatment, and prognosis throughout transitions in serious illness.

Seven themes emerged from the data that described how cultural factors influenced family transitions in serious illness, from diagnosis up to the EOL period. The influence of family and kinship was described by themes: (1) family takes care of family, and (2) we're "going to fight this." The influence of knowledge systems was described by the themes: (3) "Seeing" and experience was believing; and (4) Families "figured it out." The influence of community/social systems was described by the themes: (5) Community eased the family burden; and (6) Health professionals both eased and hindered family transitions. The influence 
of faith/religion on family transitions was described by the theme: (7) "God was always in the background."

\section{Family/Kinship}

This study found that family/kinship was a strong influence on family behaviors and decisions throughout the illness trajectory. From diagnosis forward, family overwhelmingly described family takes care of family. Family members assumed multiple roles throughout the illness trajectory that were dictated by a sense of kinship as well as more pragmatic reasons, such as proximity to the ill person and availability to be involved. Roles included primary caregiver, decision maker, advocate, and/or support person. Family members who assumed roles of primary caregiver and/or decision maker, along with the ill person were in the "inner circle" throughout transitions. These caregiver/patient dyads were typically involved in all aspects of care from the point of diagnosis up to the EOL period. Other family members played supportive roles throughout the illness with more active involvement at specific times in illness, such as at diagnosis, during spikes in illness, and as the ill person declined in health and required more care. Supportive roles included providing respite care, transportation, listening and providing input and advice to the "inner circle." As advocates, family members questioned health professionals and practices, and challenged the status quo when they felt it was necessary. Throughout transitions, the ill person also took care of family members by sparing them emotional and physical burden. This was most evident when families experienced complicating circumstances that would divert attention away from the ill person.

Complex family compositions sometimes spurred circumstances that complicated transitions. Family compositions included nuclear, blended, extended, and estranged family members. Families were large in number and often spanned multiple generations. Illness did 
not occur in a silo, and participants described having to navigate complex and at times tragic situations with other family members that compounded the transitions they went through with their seriously ill relative. Examples of complex situations included trauma, illness and/or death in the family, or family discord, jealousy, or broken family relationships that increased strain on the family members throughout the illness. Family circumstances complicated transitions by increasing family duress and diverting attention away from the ill person to attend to "other stuff." In spite of complicating circumstances, the belief that family takes care of family prevailed as participants navigated other circumstances in addition to the serious illness of their relative.

Family takes care of family could be compared to an intricate dance that began upon diagnosis and continued across the illness trajectory (Edwards et al., 2012). The ill person typically had one or two primary "dance partners" or caregivers throughout the "dance." At times, other family partners would cut in to relieve the partner or to "dance" with the ill person in order to give the caregiver respite, offer specific skills or expert knowledge, and/or to have personal time with the seriously ill relative. Family who acted in supportive roles stayed in close proximity to the "inner circle" and were ready to cut into the "dance" when needed. Like transitions in serious illness, the "dance" kept going on and the "dancer" or ill person was always supported. At times, family members "danced" with each other or others outside the family circle to help them make sense of illness, gain support or come to a decision about treatment.

Participants also described the belief we're "going to fight this" throughout transitions in serious illness. This belief was evident from the beginning stages of diagnosis and persisted throughout serious illness transitions for some participants. This belief shaped attitudes about serious illness, what types of care was sought and when it was sought, treatment goals, and 
decision making. Participants described not accepting poor prognosis at the onset of illness, choosing to "fight" instead. Many participants "fought" the illness by encouraging the ill person to undergo aggressive therapies or participate in rehabilitation services to improve health. The attitude to "fight" was sometimes lessened by the realization of poor prognosis or due to the wishes of the ill person to cease the "fight."

\section{Knowledge Systems}

The ways that family came to know about or make sense of the illness transition could be likened to "instructions" for the "dance." Participants described how they knew and what they came to know about the illness, treatments, and prognosis from the point of diagnosis up to the EOL period. For families "seeing" and experience were believing. Family members made sense out of initial diagnoses, changes in the seriously ill person's condition, or treatments based on what they observed or what they knew from previous experiences with family, friends, education, or clinical practice. Visible clues such as "seeing" the ill person function normally often led participants to believe that they were "ok" and therefore, not seek treatment or care. Conversely, "seeing" the ill person decline physically or not function normally, or witnessing poor diagnostic results, led family members to seek out care and sometimes led to the realization that the ill person would not recover in spite of medical interventions. "Seeing" was sometimes in conflict with what family members had been told or led to believe by health professionals. This sometimes led families to seek care elsewhere and caused distrust of health professionals.

Previous experience shaped family understanding of the illness and treatments and decisions that were made about care. Family members who were not health professionals described care choices that were based on experience of illness with other relatives. For example, ill persons and their family chose to forego treatments because of previous experiences 
of other family with similar treatments, such as chemotherapy. These participants also described how their understanding of the disease prognosis was initially based on previous experiences. These previous experiences provided a foundational understanding of and expectations for what might transpire. Those participants who were health professionals described similar ways of understanding illness, treatment, and prognosis that came from personal experiences with illness or clinical practice and/or formal education. Furthermore, family who were health professionals were turned to for information and/or advice throughout transitions in illness, particularly when new symptoms, diagnostic tests, or treatments were introduced. Rather than turn to external care providers for advice, family members chose to look within their family circle to get answers. Both lay and health professional participants were challenged throughout the transition to think differently and re-evaluate situations when they learned information that was in opposition to their previous experiences and/or education. Participants without previous personal or professional experience with illness were relatively naïve to the experience and initially had little or no point of reference for what they were up against.

Throughout the illness, family members "figured it out” by talking about it and educating themselves. Families looked within their trusted circle to "figure out" what was going on. This circle included the patient and caregiver dyad, as well as more peripheral support persons, those family on the periphery of the inner circle. Family conversations took place in the absence of health professionals, and both included and did not include the ill person. Within these conversations, families used the knowledge and resources available to them in order to "figure out" changes seen in the ill person or what new information meant. Conversations allowed families to "figure out" what to do next or led to the realization of poor prognosis. 
Participants also "figured out" what was going on throughout transitions by educating themselves, which included looking up information on the Internet, asking questions of health care professionals, and asking questions of friends and family members who were in the health professions. Self-education took place when family members were presented with unfamiliar information or didn't understand what they were "seeing" in the ill person. Families were resourceful, and did not explicitly rely on health professionals to provide them with needed information. In fact, health professionals were often turned to for answers after family members had sought information elsewhere. Furthermore, families would sometimes seek out information when they were unsure about what health professionals were telling them, or didn't fully agree with information presented by health professionals. Self-education influenced understanding of the illness and treatments and sometimes led to decisions made about care by the ill person and their family. Despite all "instructions" for the "dance," family members were never fully prepared for the "dance." Family members expressed that going through transitions themselves with their relative was much different than anything they had heard about or seen before.

\section{Community/Social Systems}

This study found that community/social systems also influenced transitions from the time of diagnosis up to the EOL period. In this "dance" community comprised the outer circle, standing behind family in supportive roles. Community included friends, neighbors, and health professionals. Community would "step-in" to the "dance" at specific points to ease family burden. Trusted longtime friends, neighbors, and employers eased family burden during times of duress by preparing meals, transporting children to school or events, providing respite care, or simply listening to family. Times of duress included the time of diagnosis when there was extensive diagnostic or surgical interventions, during intense treatment periods, or in the end 
stages of illness, when the ill person required more physical care. In this study, community was described as an extension of the family who eased the burden by assuming day-to-day family functions so family could focus more on the care of the seriously ill person and other family. Similar to family in more supportive roles, friends and neighbors entered the "dance" and "danced" to give the family "dancers" a break.

This study also incorporated health professionals as a component of community/social systems. Findings from this study revealed that health professionals both eased family transitions and hindered family transitions through their behaviors and communication practices. Throughout transitions in serious illness, health professionals hovered outside the trusted family circle and would step into the "dance" and at times took the lead. Typically during spikes in illness, health professionals stepped into the "dance" by invitation as families sought treatment, care, support, or information. When health professionals took time with families, inquired about family needs, or attempted to help family members understand, they eased transitions. These caring behaviors helped to build trusting relationships between health professionals and families. Good communication that was in keeping with family preferences and expectations was described as a caring behavior that eased family transitions. Family members had a better understanding of the illness and were more equipped to make decisions when honest information was provided with sensitivity and attentiveness to family needs.

Health professionals also hindered family transitions in serious illness. In essence, family looked to health professionals to take the "lead" and became frustrated when professionals did not consider family needs or did not communicate effectively with the seriously ill person or family. Ineffective communication included not being sensitive to the patient and family desire or readiness for information, poor timing of communication, presenting inaccurate information, 
and/or not being forthcoming with information. Poor communication practices of health professionals influenced participants' understanding of the illness, decisions about care, and realization of prognosis. Family members described making decisions that they later regretted because health care providers were not forthcoming with information, conveyed incorrect information, or withheld information. Furthermore, participants described being frustrated with health care providers when they presented information without sensitivity to family needs. Family didn't trust health professionals who did not give honest information or did not present information in a caring manner. Lack of communication and other uncaring behaviors negatively impacted family transitions by causing undue stress, inducing uncertainty and mistrust, and/or prolonging acknowledgement of prognosis because families did not have the information that they needed.

As providers of care and informational "gatekeepers", health professionals played an integral role in family transitions in serious illness. How health professionals acted, what they said, how they said it, and when they said it mattered to families. Though only invited to the "dance" at specific points in time, health professionals' ability to "make the right moves" at the right times influenced family understanding, health seeking and caregiving behaviors, and decisions throughout illness. This finding conveys the importance for health professionals to remain attentive to and engaged with families when they are given the opportunity to participate in care. Though not part of families' inner or outer circles, health professionals are important peripheral participants in the "dance" that can and do impact how families navigate transitions.

\section{Faith/Religion}

This study revealed that for this sample, "God was always in the background". Christian beliefs and practices influenced family members' behaviors and understanding at different points 
throughout the illness. The majority of family members spoke about prayer throughout the illness, from diagnosis forward. Prayer was described as a routine ritual or in an attempt to seek healing for the ill person. Prayer increased during spikes in illness and in the later stages of illness. Three participants described relying on prayer as a way to find healing for their relative; one participant even described "laying hands on" his wife in an attempt to get healing. Prayer was a normal daily ritual that held different meaning and purposes at different points in time across the illness continuum. For some families, prayer was a daily ritual that family did as part of their routine. In those families, religion and faith played a more passive role in the serious illness transition. For the most part, God was in the background, and families "prayed and stuff" but it only came to the fore during times of crisis. For others it was an active testament to their faith and "handing" things over to God throughout the family transition.

Participants also described struggling with their faith as they witnessed suffering. When family members witnessed pain and distress in their relative, they struggled with their faith. Family members couldn't understand how their loving God could allow such pain and suffering in the ill person. Participants who described this inner turmoil were also the ones who professed the greatest reliance on faith throughout transitions in serious illness. Because their faith was so great, they had extreme difficulty coming to terms with the suffering of their relative. The intimacy of relationships between the family member and the ill person could partially explain this struggle; participants described close bonds with their seriously ill relatives. Participants grieved for their relatives long before their death. Furthermore, participants who were health professionals struggled to maintain faith in God's healing power because of their formal medical and nursing education. They had trouble balancing knowledge based on science with what they had been taught and believed about faith and religion throughout their lives. Personal beliefs 
conflicted with professional values. Statements about families struggling with their faith were profound, and provided insight into the potential moral distress that families may experience as they bear witness to suffering of loved ones or try to balance scientific understanding with faithbased beliefs.

\section{Study Findings Related to Conceptual Framework}

This qualitative descriptive study was grounded by the concepts of transitions in illness (Meleis et al. 2000; Edwards et al., 2012; Penrod et al., 2011) and culture. The specific transition examined by this study was the time spanning from the point of diagnosis up to the EOL period. The specific cultural factors of interest were family/kinship, knowledge systems, community/social systems, and faith/religion (Leininger, 1967; 1988; Whitehead, 2008; Vandenberg 2004). This section discusses how findings from the study both support and add to the conceptual understanding of transitions in serious illness and culture.

\section{Transitions in illness}

This study provides new insight into family transitions from the time of diagnosis to the EOL period, an understudied serious illness transitional time period. In this study, transitions began when the family learned of the diagnosis of the ill person, spanned the time period when families and ill persons actively sought medical care, and ended when families realized that the ill person would not get better as a result of medical treatments. Families described the transition as having ebbs and flows that were punctuated by periods of intensive treatment, times of wellness, spikes in illness accompanied by new diagnoses, periods of uncertainty, and gradual decline. This study expanded on what was known about family transitions in illness by examining transitions further upstream in illness; most prior studies looked only at family 
transitions experienced in the EOL phase (Bourgeois \& Johnson, 2003; Spichiger, 2008; Phillips and Reed, 2009; Csikai and Martin, 2010; Donovan et al., 2010; Duggleby \& Berry, 2005; Duggleby, et al.,2010; Kreling et al., 2010; Waldrop et al., 2005). Few studies have addressed family transitions from earlier points in the illness trajectory. Those studies that did look at family transitions across the illness trajectory only examined models of caregiving and decision making between the ill person and his/her primary caregiver (Edwards et al., 2012; Penrod et al., 2012). This study explicated the many ways that multiple family members, including caregivers and support persons, navigated transitions in serious illness. Furthermore, this study described how cultural factors shaped family transitions across the illness trajectory and looked at more than just caregiving and decision-making.

This study found that transitions are mediated by culture (Meleis et al., 2000; Meleis, 2010; Leininger, 1988). Findings from this study revealed that the cultural factors of interest influenced families' understanding of illness, decisions about care, role assumptions, behaviors and actions, and realization of prognosis. Though all cultural factors were described as influential throughout illness transitions, there were variations among participants in when and how cultural factors came into play.

Previous theoretical work compared decision-making in the context of advanced cancer between the ill person and his/her primary caregiver to a dance (Edwards et al., 2012). These two persons "danced on the stairs" throughout the illness to make decisions about care. Movement up and down the stairs occurred when new information was presented by health professionals or when new treatments were introduced (Edwards et al., 2012). Throughout the dance, the ill person and their primary caregiver remained engaged with each other to make sure 
that decisions were made that were in alignment with the ill person's desires. The dance began at diagnosis and ended after the death of the ill person (Edwards et al., 2012).

In this study, transitions in serious illness could also be likened to a "dance" with various "dancers" and participants that came and went. In this study, dancers were more than the ill person and primary caregiver. Other family members, community, health professionals, and God were also included in the dance. Family dyads that involved the ill person and their primary caregiver comprised the main couple in "the dance." From diagnosis to the EOL phase, these "partners" or inner circles worked together to complete the "dance" or navigate the transitional ebbs and flows. The true power of decision-making and care burden rested with this dyad or inner circle. Other "dancers" moved in as necessary throughout the "dance." Supportive family members remained in the outer periphery of the circle and stepped into the "dance" as needed to provide support, provide respite care, or help figure things out. Though not as integral to decisions and caregiving, this "outer circle" of family played important roles in providing physical and emotional support to both the ill person and the primary caregiver, and were looked to for information and advice to help "figure things out" throughout illness transitions. Family in both the inner and outer circles were the most trusted persons throughout transitions. Before turning to others, family turned to each other for support, information, and guidance.

Outside of the family circle were community and health professionals. Community friends and neighbors stepped into the "dance" during times of duress and eased family burdens by providing respite for day-to-day activities. Friends and neighbors typically had long-term relationships with families and were trusted by families to take over day-to-day tasks so that families could tend to the needs of the seriously ill person. They were not included in health conversations or decisions about care. Health professionals were invited to "the dance" by 
family at specific times to provide treatments or information to the ill person and their family. Health professionals eased transitions and made transitions more difficult. Health professionals influenced decisions made by how they communicated information to the family and through caring or non-caring behaviors that they displayed towards the family. Many times, health professionals didn't communicate information to the family. This led to frustration among family members. Furthermore, family members would turn elsewhere to educate themselves about serious illness transitions when they felt that health professionals were not meeting their needs. Trust and inclusion of health professionals was largely dependent on how health professionals communicated with family and the caring behaviors they did or did not exhibit.

Throughout the "dance" families drew information from a variety of knowledge systems to help them make sense of transitions in serious illness. These knowledge systems could be thought of as the "dance instructions." How families came to learn the "dance" was largely independent of health professionals. The "dance" was learned from previous "dances" or from watching others "dance" with serious illness. When families couldn't figure out a "move" they sought out answers and educated themselves. Families were resourceful, and health professionals were turned to when answers couldn't be gained elsewhere or when they were present with the family. Family members didn't always receive information or correct information from health professionals, leading them to go elsewhere for care or information. "Instructions" gained from other knowledge systems were sometimes incongruent with information presented by health professionals, leading to uncertainty and mistrust among family members.

Throughout the "dance" God was omnipresent. For most participants, faith and religion was an umbrella, under which the "dance" took place. Families turned to faith and religion for 
ritual and comfort. For others, God was an intimate partner in the dance and was prayed to for healing of the ill person. These family members experienced great distress and inner conflict when prayer didn't work and they had to witness extreme suffering in their ill relative. Their beliefs were challenged, yet they still felt compelled to place complete faith in God. Family members who were also health professionals faced a similar dilemma. Their knowledge gained from formal medical or nursing education was sometimes in conflict with lifelong beliefs to "trust in God." These participants struggled to find a balance between scientific knowledge and religious beliefs. This led to self-doubt and feelings of guilt at times.

\section{Culture}

Findings from this study support existing theories that cultural factors shape views of health and illness (Leininger, 1988; Whitehead, 2004), yet move beyond previous work by examining specifically how cultural factors influenced family transitions in illness. Findings from the study revealed family/kinship, knowledge systems, community/social systems, and faith/religion influenced family understanding of illness, decisions about care, role assumptions, burden of care, interactions with care providers, and realization of prognosis from the point of diagnosis up to the EOL phase. Cultural factors came into play at different points throughout illness transitions, and the importance of each factor varied throughout transitions. For example, participants described the importance of community as a support system during spikes in illness, while in the more dormant stages of illness, community wasn't mentioned as a influencing the families' thoughts or actions related to the illness.

This study expanded on ideas of what comprises each cultural factor. Community, traditionally understood as friends and neighbors (Leininger, 1988), was expanded to include health professionals and institutions. New insights into the many ways that family members 
knew or came to know and understand throughout the illness were revealed, including "seeing" changes in the ill person and previous experiences. Previous experiences included not only formal education, but experience with illnesses of others as well. Furthermore, family conversations that did not include health professionals were ways that families came to know and understand different aspects of the serious illness and its treatment options. Trusted ways of knowing were described. For example, families typically trusted experience and "seeing" more than they trusted information presented by health professionals. This may be in part because information gathered from health professionals was in contradiction to information gleaned from other ways of knowing. Additionally, health professionals were not always forthcoming with information. All of these knowledge systems influenced family understanding, behaviors, and decisions at multiple points throughout the illness trajectory.

Unlike many previous studies, this study supports more recent ideas on intra-cultural variation, which state that in spite of cultural similarities, there are individual variations in how persons assimilate life experiences (Whitehead, 2004; Vandenburg, 2008). Persons within the same cultural group have individual beliefs and values that shape their interpretation of life events. In this study, each of the four cultural factors shaped individual family transitions in illness slightly different. Though family took care of family, within this sample families were complex, and each family structure was different. Furthermore, though there were similarities in how roles were assumed within families, in no two families were roles assumed exactly the same way. Intra-cultural variations were evident in how faith and religion influenced family transitions as well. For example, for the entire sample, throughout transitions, "God was always in the background." The majority of participants described God, religion, and faith as influential to illness transitions, yet how and when faith and religion influenced family 
transitions in illness varied across the study sample. Some participants described faith/religion as an important way to seek healing for their relative throughout the illness, while others described faith/religion as a ritual or practice that they performed at specific points in time throughout the illness trajectory. Variations in how cultural factors shaped family transitions in serious illness among participants in this sample supports the idea that there is no one way to provide care to persons experiencing similar life events. It cannot be assumed that all persons within a cultural group believe, behave, or interpret information in the same way.

\section{Findings in Relation to the Literature}

\section{Family/Kinship}

Families, in all of their complexity, are involved throughout transitions, but in different ways, at different points in time. Previous studies offer limited insight into how complex family systems make sense of and navigate serious illness together primarily due to their focus on the role of primary caregiver during the late stages of illness (Dalgaard et al. 2010; Duggleby et al., 2010; Edwards et al., 2012; Pinnock et al., 2011). As this study revealed, family is more than just the primary caregiver. Families are complex social organizations that band together to take care of their own. Within families in this study, there was an "inner circle" of persons who assumed primary caregiver and decision-making roles throughout the illness. The "inner circle" always included the ill person, and there were informal rules as to who else was in the "inner circle" and why; primacy of relationship, feelings of devotion, and pragmatic rationale typically dictated who was in the "inner circle." This "inner circle" or family dyads were the main partners of "the dance" and were largely responsible for decision-making throughout the disease trajectory. Other family members entered the "dance" as needed. These family members dwelled in close periphery to the "inner circle," creating an "outer circle" of support. They 
entered and exited the "dance" as needed to provide physical and emotional support to the main “dance partners." Patterns of family involvement were dependent upon the illness and need for direct caregiving and support, but also complicating matters, such as such as conflicting family obligations and family loss and tragedy. The "dance" was sometime interrupted by these other life events, and family members had to "step away" from the "dance" temporarily to tend to other matters. Overwhelmingly, it was evident that illness was a family affair in which everyone was involved, and family takes care of family. All family members were present for the "dance" but entered and exited the dance at different times. These findings emphasize current standards to include families in care of the seriously ill across care settings (American Nurses Association, 2010). Additionally, these findings offer insight into who is considered to be "family" and who could and should be included in care throughout illness trajectories.

This study extends our understanding of family conflict over EOL measures by illuminating the many complicating circumstances that occurred throughout serious illness, as well as how families navigated these circumstances. Families did not experience the illness of their relative in isolation, rather families had to navigate other complex situations while also transitioning in illness with their family member. This complicated transitions and created strain and discord within the family. Findings revealed that family discord and caregiver strain are not limited to the EOL phase of illness (Carrion \& Nedjat-Haiem, 2013; Lockie et al., 2010; Waldrop et al., 2005; Winter\& Parks, 2008), but span across the illness trajectory and affect multiple family members as they navigate and negotiate care for their relative. Furthermore, the ill person was often privy to complicating circumstances and tried to spare their family additional stress and worry when possible by not sharing critical information or choosing care to in order to avoid burdening family members. This was to the disappointment of family members who 
didn't understand why the ill person “just didn't tell us.” Furthermore, family members preferred to know vital information that the ill person withheld so that they could be more prepared for transitions. These complicating circumstances may contribute to the reported emotional, physical, and psychological distress of family members after the death of the ill person as they reflect on decisions made and care provided, (Braun, Beyth, Ford \& McCullough., 2008; Meeker \& Jezewski, 2005; Paul \& Rattray, 2008; Phillips \& Reed, 2009) and wonder if they could have been more attentive to the ill person throughout the serious illness.

In this study, family members expressed beliefs and attitudes of we're "going to fight this," regardless of diagnosis. This belief has been associated with cancer in the past. Positive thinking has been perceived to be responsible for a more positive outcome or cure of the disease (Hurley, 2014; Ruthig, Holfeld \& Hanson, 2012). Regardless of diagnosis, in this study, fighting behaviors included pursuing aggressive treatment, undergoing rehabilitation, and rejecting hospice services. This belief may have prolonged transitions by leading family members to pursue curative measures even though they were medically futile. For most participants in this sample, realization of EOL came very late in the disease trajectory, within days to weeks of the death of the seriously ill person. Ceasing curative treatment and accepting hospice was described as accepting death and "giving up." Rejection of hospice prevented families from receiving patient-centered, family-focused, holistic services that could help to alleviate suffering experienced by the ill person as well as physical and emotional distress experienced by family members. Such beliefs and attitudes may help to explain the reluctance of patients and families to enter into palliative and/or hospice care programs or to forgo medically futile treatments. 


\section{Knowledge Systems}

This study expanded on understandings of how families know and come to understand illness transitions from the point of diagnosis up to the EOL phase. This can be compared to “instructions for the dance." This study revealed that family members relied on multiple knowledge systems throughout illness transitions to understand the illness and make decisions, including the "seeing" changes, past experiences and education, and educating themselves by talking about it and seeking out answers. Furthermore, this study described when families turned to these systems, and how these systems influenced actions, understanding of illness and care decisions at specific points throughout illness transitions.

In this study, "seeing" and experience were truly believing. In spite of what family were told by health professionals, visible clues and previous experiences influenced family members' understanding of illness, behaviors, and decisions from the point of diagnosis up to the EOL period. In this study, family members described observable clues such as changes in appearance or function of their relative that signified illness or wellness. Family members described seeking care when they saw changes in their relative, as well as not seeking care when everything appeared normal. Furthermore, though family members had knowledge of illness and the potential poor prognosis of disease, realization of the severity of illness was largely influenced by changes that family members did or didn't see in their family member. This was even true even among health care professional participants. Family members also came to realize the impending death of their seriously ill family member because of changes that they could see. The notion that seeing is believing is of utmost importance for health care professionals to recognize as they deliver care to patients and families alike. It provides valuable insight into how 
persons with serious illness and their families view wellness, make sense of illness, and why and when they do or do not seek care.

This study advances the understanding of the influence of previous experience on illness transitions as well. In this study, experience was derived from health situations, observations of and participation in care of family and friends, and professional education and practice and influenced how family members transitioned in serious illness. Family members' baseline knowledge of the illness upon diagnosis was based on such previous experience; what family members thought about the illness and what to expect as the illness progressed came from previous experience. Though the literature on the influence of past experience on transitions in serious illness is scarce, authors have discussed the implications of past experience on choice of care providers. Similar to one participant in this study, another qualitative study described how patients and relatives experiencing deterioration in health lost confidence and avoided future contact with care providers that were inattentive to their health care needs (Rainey, Ehrich, Mackintosh, Dip, \& Sandall, 2013). Yet, this study found that past experience also influenced understandings and viewpoints of illness, decisions about care, trust and interactions with health care providers. Past experiences shaped the attitudes, behaviors, and decisions of family members as they transitioned through illness. Attention to the knowledge and experience that family members bring into the illness is necessary to understand the choices and behaviors of families and to help prepare families for what to expect throughout transitions in serious illness.

This study also captured the influence of formal medical or nursing knowledge on transitions in serious illness. These findings have not been reported in previous literature. As previously noted, four participants were in the health professions, thus had formal medical or nursing knowledge that influenced their understanding of illness and roles within the family 
throughout illness transitions. Interestingly, in spite of their knowledge of the illness, treatments, and prognosis at the onset of illness, the majority of these participants described not being prepared for the experience and not knowing what to expect throughout the course of the illness. These findings reveal that in spite of formal education, health professionals that are also family of persons with serious illness have emotional responses throughout transitions that influence reckoning of illness that may be in opposition to their nursing or medical knowledge. Additionally, these persons experienced being caught between the role of family member and health professional, as they were often turned to for advice or information from other family, while they were also trying to make sense of transitions themselves. These findings provide insight into the complexities of a sub-culture that has unique needs when transitioning in illness.

Family members displayed remarkable resourcefulness as they "figured out" transitions. Family members used the Internet to educate themselves about the illness and gain a better understanding of "what they were up against." This is concurrent with current literature that describes the use of the Internet for health information (Dolce, 2011). Internet usage to gain knowledge may have implications for families, patients, and care providers alike. In a previous qualitative descriptive study, researchers found that patients and their families often became disenchanted with health care providers because of information that the patient and family gained from the Internet that was not consistent with information presented by their healthcare provider. Furthermore, as a result of information gained from the Internet, patients and families felt empowered to challenge health care providers on treatments or change care providers when they felt that they were not receiving appropriate care (Dolce, 2011). When families receive information that is incongruent with their understanding or when physicians are not forthcoming 
with information, families will seek out information to make sense of transitions in serious illness.

One unique way of coming to know in this study was intra-family communication, or "talking about it." Family members talked about it at critical points and times throughout the transition, such as upon diagnosis, when new treatments were introduced, and when spikes in illness or declines in health were noticed, in order to figure out what was going on with their ill relative or come to a decision about treatment. Communication patterns within families influenced not only knowledge of illness and treatments, but realization of prognosis as well. Care conversations allowed families to have a better understanding of illness, treatments and prognoses, and facilitated decision-making. Although previous research has discussed the importance of nurse-driven family care conferences in chronic illness (Benzein, Olin \& Persson, 2015), this study is unique because it describes how families had conversations to "figure it out" without the assistance of health professionals. There was no guide or moderator to fill in gaps of knowledge or answer questions. Families "figured it out" on their own, and made decisions based on what was communicated within the family. Also, in this study, families who "didn't really talk about it" had incomplete information, had more difficulty coming to terms with the illness, and were less prepared for illness transitions. Talking about it converted knowledge to realization, as families described realizing that “we probably didn't have as much time as we thought we had" as a result of family conversations in the later stages of illness transitions. Communication within families is an important mechanism that families employ to understand the illness, treatments and prognosis throughout transitions in serious illness. 


\section{Community/Social Systems}

The current study highlighted the importance of community members throughout illness transitions as extensions of the family who were available to assume day-to-day responsibilities for families so that they could focus more on the care of the seriously ill person. Trusted neighbors and friends entered the "dance" in times of duress. The provided respite care or were trusted with day-to-day activities to relieve the burden on the family. Previous research has discussed community as a good support for EOL family caregivers (Duggleby et al., 2010; Lockie et al., 2010), however, this study further described specific points from diagnosis up to the EOL phase when community members were most helpful to all involved family members. Points included the time of diagnosis, when there was extensive diagnostic or surgical interventions, during intense treatment periods, or in later stages of illness, when the ill person required more physical care. Community members are trusted by the family. They can ease the family burden at multiple points along the illness trajectory rather than just in the end of life phase.

Unlike previous studies, this study included health professionals as a component of community/social systems. Health professionals both eased transitions and complicated transitions. Health professionals eased transitions through demonstrations of supportive behaviors and good communication practices. Appreciation of patient-centered, family-focused care was evident in the current study. Families described feeling cared about and supported when health professionals took time to connect with them, listened to them, and tried to help them figure things out. This is consistent with findings from previous studies, where EOL caregivers trusted health professionals that were caring and compassionate, and individualized care to their specific needs (Duggleby et al., 2010; Powers et al., 2011; Tallman, 2012). Findings 
from this study also echoed findings of previous studies, which found that families valued honest, timely communication by practitioners (Bernard-Dubenske et al., 2008; Csikia \& Martin, 2010; Dalgaard et al.,2010; Duggleby et al., 2010; Powers et al., 2011; Tallman et al., 2012; Russ \& Kaufman, 2005). Furthermore, this study found that family members had different preferences for when and how information was communicated by health professionals. In addition to providing honest information, families desired to have information presented at times when they were ready to receive it. Not all family members wanted to be told of poor prognosis upon diagnosis. These family members still wanted to "fight" the illness and were not ready to "give up" on their relative. These family members presumed that health professionals were "giving up" on their relative by delivering a poor prognosis upon diagnosis, and as a result of this came to distrust that health professional and seek help elsewhere. Others wanted health professionals to be forthright with them at all stages of the illness. These family members desired to know up front what they were facing so that they could get their affairs in order and make the best decision for themselves and their relative. Attention to family needs, preferences, and communication styles can ease transitions by allowing families to feel cared about and have a better understanding of the illness, treatments, and prognosis.

Health professionals also complicated transitions by not being attentive to family needs, not communicating effectively with family members, and not knowing what to do. Health professionals did not always acknowledge that not all individuals share the same abilities to hear and process information, and did not pick up on family cues for readiness for communication. In this study, communication of poor prognosis was typically done in the very end stages of illness, when all curative measures had been exhausted. Family members often wished that this information had been conveyed earlier. Initiation of advanced planning conversations are 
recommended at any age and state of health, yet families and healthcare providers alike have described not initiating such discussions because they were uncomfortable doing so (IOM,2015:Zeytinoglu, 2011). Findings from this study suggest that there is no one ideal time to deliver prognostic information, rather health professionals must be attune to the needs and desires of seriously ill persons and their families throughout transitions. The best way of learning about family preferences is to ask. Attention to the needs and desires of families transitioning in all stages of illness is necessary to provide welcomed care and communicate effectively.

\section{Faith/Religion.}

This study found that in addition to helping to provide comfort to family members in the end stages of illness, faith and religion could also cause a great deal of inner turmoil for family members as they struggled to understand how God could allow such horrible suffering of their relative. Previous work has identified how faith and religion as help families to have more confidence in decisions in the end stages of illness (Donovan et al, 2010) and find closure in the end stages of illness (Bourgeois \& Johnson, 2001), but has not discussed the potential moral distress families might experience as they struggle to understand why God has allowed suffering to occur. Suffering was witnessed at multiple points throughout the illness trajectory, typically during spikes in illness or as the illness progressed to the later stages. Furthermore, because this study included health professionals, for some participants, there was a struggle to balance religious/faith beliefs with scientific knowledge when trying to understand and come to terms with their relative's illness. What family members knew because of scientific knowledge was in conflict with their belief that God could intervene and provide healing. 
Finally, this study advance previous work by describing variations in when and how faith and religion come into play at throughout serious illness transitions for individual families. No one family relied on religion and faith in the same way; while some families described "praying" at various times throughout the illness, others looked toward their faith for healing and miracles throughout the illness. Faith and religion's influence on family transitions in serious illness is complex; though always present, faith and religion had different meanings for family members and therefore influenced family transitions in serious illness differently.

\section{Implications for Nursing}

\section{Practice}

The findings from this study have improved nurses' understanding of how cultural factors influence family comprehension of illness, behaviors, and decisions throughout transitions in serious illness, from the time of diagnosis up to the EOL phase. Nurses can use information gleaned from this study to incorporate cultural factors into the care of the family to facilitate transitions in serious illness and help families meet desired outcomes. A more in depth discussion of how nurses can take the knowledge gained from this study to provide culturally competent, patient centered, family-focused care to families transitioning in serious is included in the passages that follow.

Family/Kinship. Nurses should be prepared to involve multiple family members in the care of the ill person from the point of diagnosis on, and recognize that families are also care recipients (American Nurses Association, 2012; World Health Organization, 2007, 2012; The Joint Commission, 2012; National Hospice and Palliative Care Organization, 2013). Upon diagnosis with serious illness, assessment of who will be involved in illness transitions and to what extent 
family will be involved is needed. Such assessments may be needed throughout illness, as family involvement may change over time due to a variety of circumstances. Nurses should be cognizant that other complicating circumstances may occur concurrently with family transitions in serious illness, and may take family member's attention away from the ill person or change who assumes roles. Assessment of family structures, roles, responsibilities and extenuating circumstances allows nurses to help prepare families for transitions in illness by identifying who should be included in care conversations, when and how family members should be included, and when and how families might need help throughout illness transitions. As noted in this study, family beliefs and attitudes about illness also influence understanding of illness, behaviors, and decisions about care. Furthermore, the ill person's wishes and desires should be assessed and communicated to the family so that family members are cognizant of what the ill person feels is appropriate to share with the family about his/her condition and what he/she is willing to undergo to obtain healing. Family beliefs and attitudes towards illness and treatments should be assessed so that nurses and other health professionals can incorporate family beliefs into plans of care.

Knowledge systems. Upon diagnosis with serious illness, nurses should assess the families' current knowledge of and past experience with illness. Findings can be used to guide patient and family education and nursing care. While showing respect for patient and family beliefs and understandings of illness, nurses can also acknowledge and tactfully dispel incorrect knowledge gained from past experiences, and help families navigate current transitions. In spite of what families know or come to know throughout the serious illness, nurses must recognize that understanding and interpretation of serious illness may be influenced by emotional reactions as well. In this study, even health professionals remarked about being "unprepared" for 
transitions despite their medical or nursing training because of the emotional connection that they had with the seriously ill person. Nurses should allow time for families to come to terms with information, regardless of past experience or education. Nurses should also pay careful attention to visible clues in the ill person that influence families' interpretations of illness. While some clues may help the family come to the realization of poor prognosis, other clues may dissuade the ill person and their family from following up with medical care or seeking help when the ill person "appears" well. Family recognition of visible clues in the ill person may also present opportunities for the nurse to discuss integrative palliative care. Introduction of integrative palliative care would allow ill persons and their families to receive patient-centered, family-focused, holistic care while still undergoing curative measures. Palliative care could increase throughout the disease trajectory, and segue into hospice care later in the illness trajectory if desired by the ill person and their family.

Nurses can also assess how families educate themselves throughout transitions in serious illness. Consistent with findings from this study, the Internet has been touted as a resource for health information in recent literature. Nearly $61 \%$ of Americans have sought out health and/or medical information on the internet (Fox \& Jones, 2009). Nurses can help patients and families identify and select credible Internet sources, and interpret information derived from the Internet (American Association of Colleges of Nurses, 2013). Patients and families should be encouraged to use appropriate Internet sites to seek information and to ask questions to their health care providers to clarify information or bridge knowledge gaps. Nurses can help families determine appropriate questions to ask physicians and other health professionals, and coach families on the most appropriate ways to communicate with health professionals in order have care needs met. 
Nurses and other health professionals should be aware that families often "figure thing out" independently of care providers. Conversations within families help them to understand transitions in serious illness and come to decisions about care. Nurses can coach families on ways to converse with each other and when it might be appropriate to have certain care conversations. Nurses can also look for opportunities to participate in conversations with families. Previous literature has shown that nurse facilitated care conversations early in the illness trajectory may have positive effects for families by enabling them to have a better understanding of illness, treatments and prognosis (Benzein, Olin \& Persson, 2015). Nurses should assess families' desire for such conversations at multiple points throughout transitions in serious illness and intervene as appropriate. For example, upon diagnosis the nurse might ask the family if they would like to talk about the diagnosis and what it means to them. Throughout the diagnosis, as families are presented with information about diagnostic exams, treatments, and medications, nurses might offer to talk with families about what to expect. Finally, nurses could speak with ill persons and their families about any physical or functional changes they are experiencing and help patients and families explore possible reasons for changes or discuss which changes might necessitate medical intervention.

Community/Social Systems. Friends, neighbors, and other community members should be included in care throughout the illness trajectory as desired by families. As found in this study, close friends and neighbors served as proxy for the family, especially in times of duress, and assumed day-to-day responsibilities so that the family could attend to the needs of the ill person. Upon diagnosis and then throughout illness, nurses can assess community support and resources available to ill persons and their families, as well as the families' comfort level with utilizing resources. Nurses can help families plan ways to integrate community into care before their help 
is needed. Effective use of health care resources is essential to deliver quality care and achieve optimal care outcomes (American Nurses Association, 2012). Referrals to specialist in community resources, such as social workers, may be useful and/or necessary if there are resource gaps or financial difficulties.

In this study, families described behaviors of health professionals that helped them to feel supported and allowed them to develop trusting relationships with practitioners. Behaviors such as listening, caring, taking time, and being honest can be emulated by nurses to build rapport with patients and families alike. Throughout the illness trajectory, health professionals should treat families with dignity and respect. Family needs, concerns, and questions should be listened to by nurses and given the appropriate time and resources needed to fulfill needs, alleviate concerns, and answer questions.

Communication styles of nurses and other clinicians influenced family transitions in serious illness. Honest, forthright delivery of information should be done in a timely manner, yet with sensitivity to patient and family needs. For example, as seen in this study, it may not be appropriate to introduce hospice as the only care option upon initial diagnosis with an illness. Families may need time to digest and process information and come to terms with the situation before making such decisions. Attention to patient and family cues for readiness for information is needed as this study revealed that readiness for conversations may vary between families. Conversations should occur when the family exhibits readiness.

Faith/Religion. Religion often plays an important role in rural communities. In this study, participants almost uniformly expressed that "God was in the background," however, there were variations in the importance and timing of the inclusion of faith and religion throughout illness transitions. Faith also caused inner conflict for some participants, who struggled to come to 
terms with how God could allow suffering in their family member. These findings suggest that inclusion of faith and religion in illness transitions is an individual preference. Determination of individual family religious and faith-based practices are needed to provide quality care, therefore nurses should explore the religious beliefs of families, incorporate desired practices into care, and find ways to help families come to terms with internal conflicts that result from such beliefs if necessary. Referrals to clergy and other religious leaders may be needed.

\section{Education}

Nursing curricula should expand on ideas of what is culture. Nurses need not only to have an understanding of and respect for different races and ethnicities, but also be attentive to other cultural and socio-economic factors that might influence behaviors and attitudes (IOM, 2014; Leininger, 1988; Vandenberg, 2010). Faculty must incorporate theories of what shapes culture into the curriculum so that students have a better understanding of how and why culture is formed (Leininger, 1988; Whitehead, 2004). Curricula should stress an attitude of cultural acceptance and willingness to adapt to others' needs (Dudas, 2012), as patients, families, and cultural groups will most likely reject care that is not in alignment with their beliefs (Leininger, 1988). In addition to this, curricula must also stress the complexity of culture and the many intra-cultural variations that students will experience within similar populations. This study found that family/kinship, knowledge systems, community/social systems, and faith/religion influenced family transitions in illness, yet variations in how cultural factors influenced transitions in illness were noted. For this reason, students should be instructed to listen attentively to the needs of ill persons and their families and assess individual preferences for care. Finally, curricula should assess students on caring behaviors that are sensitive to the 
cultural needs of the patient population (Dudas, 2012), yet also attentive to individual and family preferences.

Nursing curricula should prepare students to provide patient-centered, family-focused care across the lifespan and across the continuum of healthcare environments (AACN, 2010; ANA, 2012). As seen in this study, transitions in serious illness occurred both in and outside of institutional settings, spanned long periods of time, involved multiple family members, and were influenced by cultural factors. As a result of these findings, students should be encouraged to incorporate family into care much further upstream in the illness trajectory, rather than just in the end stages of illness. Furthermore, patient-centered, family-focused approaches to care should be stressed by educators. Such care includes not only attention to culturally mediated needs, but also takes into consideration that each patient and family is different and may have unique needs. Student instruction in assessments that identify unique needs is necessary. For example, based on findings from this study, students would be taught to use ecomaps to assess family structures, family roles, and past experience with illness. Students should be taught to derive patientcentered, family-focused interventions from assessment findings.

It has been noted that health care professionals do not feel that they receive adequate education or practice discussing sensitive information with patients and families (IOM, 2015; Sanders, 2015; Wittenberg, Goldsmith \& Ragan, 2011). This study described how communication practices of health care professionals both positively and negatively influenced family transitions in illness. Families described the need for honest, forthright provision of information delivered by caring, sensitive professionals. Furthermore, findings from this study pointed out that families are ready for information at different times throughout the serious illness trajectory. Schools of nursing and other health related disciplines need to develop 
curricula that support caring, honest communication of illness, prognosis and treatment options, yet are sensitive to family desires for information. Students should be taught how to be attentive to family cues of readiness for information, and recognize that there is not one appropriate time or way to communication with families. Instruction on how to form collaborative relationships that include the ill person, family, and care providers and focus on holistic needs throughout illness rather than just at the end stages is needed (Wittenberg, Goldsmith \& Ragan, 2011).

\section{Research}

This study provided insight into how specific cultural factors influenced families' transitions in serious illness. Family members retrospectively described how family/kinship, knowledge systems, faith/religion, and community/social systems influenced actions, understandings, and decisions throughout transitions in illness. While these findings contribute to the understanding of a phenomenon about which little is known, a broader understanding of the world view of families transitioning in serious illness could be obtained through focused ethnography (Cruz \& Higginbottom, 2013; Polit \& Beck, 2012). Ethnographic methods require the researcher to collect data in real time, while the subjects are experiencing a phenomenon. An ethnographic study could expand on findings from this study and allow nurses to better provide a understand the emic view of family transitions in serious illness in real time, as families experience transitions (Cruz \& Higginbottom, 2013).

This study included descriptions of both family members who had knowledge of the illness because of formal nursing or medical training and those who did not. Though similarities in descriptions existed between the two sub-groups, differences were also noted. Those with formal medical or nursing knowledge described knowing about the illness upon diagnosis, and how that knowledge influenced understanding of transitions and attitudes towards care. 
Additionally, family members with formal nursing or medical education described inner conflicts related to playing the role of information broker to the family and coming to terms with the illness of a person to whom they had strong emotional ties. Additional research of family members who are also health professionals may be warranted to gain insight into an understudied sub-culture. Replication of this study using a more homogenous sample may provide more insight into this or other sub-cultures transitioning in illness with a seriously ill family member.

This study found that health professionals could ease or hinder family transitions in serious illness through behaviors and communication patterns. The need for health professionals to communicate with families in ways and at times that were desired by families was evident. Additional studies may be needed on communication practices of healthcare providers. Information on when and how healthcare providers initiate care conversations is needed before changes to practice can be made. Furthermore, identification of perceived barriers to communication by healthcare providers could provide insight into why care conversations happen late or not at all throughout the illness trajectory. Moreover, previous research has indicated that nurses can play an integral role in communicating with family; research focused specifically on the role of the nurse in facilitating or mediating care conversations may be beneficial. Findings from such studies could provide insight into what nurses believe their role is in care conversations and ways to expand roles.

Many potential nursing interventions geared toward easing family transitions in illness were suggested based on findings from this study. Nurses could intervene in illness transitions a variety of ways, including: anticipatory guidance, nurse-mediated care conversations, patientcentered, family-oriented education, navigation of knowledge systems, and referrals to 
specialists as needed. Interventional studies are needed to determine the efficacy of such interventions across the illness continuum.

\section{Strengths and Limitations}

\section{Strengths}

One of the major strengths of this study was the diversity of the sample. Family members that participated in the study included daughters (2), nieces (2), sons (2), husband (1), sisters (2), granddaughter (1), daughter-in-law (1), and a close family friend (1). Furthermore, participants identified with a variety of roles within the family; six of the twelve participants self-identified as a primary caregiver or decision maker for the ill person throughout the illness, while the remaining six participants identified a more supportive role throughout the illness. Ages of participants ranged from 28-73 years of age, with a median age of 53. Eleven participants were Caucasian and one participant was African American. Although there was only one person of ethnic minority in the sample, the sample was representative the homogeneity of the region (WVDHHR, 2010). Additionally, deceased family members of participants died as a result of various illnesses, including: esophageal cancer (1), prostate cancer (1), Parkinson's disease (1), ovarian cancer (1), pancreatic cancer (3), breast cancer (1), cancer of unknown origin (1), congestive heart failure (1), and aortic stenosis (1). These illnesses were representative of the leading causes of death of persons living in north central WV (WVDHHR, 2010). Findings may be transferred to similar populations, such as families of persons with serious illness, families living in Appalachia, or families in rural communities. The rich description of study participants can help practitioners decide who findings may be transferred to. In dissimilar populations, findings can be used to gain insight into how cultural factors might come into play 
throughout family transitions in serious illness. Healthcare providers could use this information to assess individual and family needs throughout transitions in illness.

Another strength of this study was adherence to research methods that produced data near results. An audit trail was maintained outlining the systemic approach undertaken by this investigator that was consistent with conventional content analysis. The research question, as well as concepts of culture and family transitions in illness were used to develop the interview guide. Interviews were conducted beyond the point of data saturation, as no new categories emerged after interview nine. The interview question as well as key concepts were used to anchor analysis of data. Similar categories were uncovered across the sample, and findings were presented in the language of the participants. Categories were named using language of participants, and sub-categories further elaborated on participant descriptions. Furthermore, member checks were conducted with three participants to make sure that findings depicted accurate descriptions of family members of seriously ill persons. Direct participant quotes were provided to support abstraction of categories.

The rigor maintained throughout this study facilitated the acquirement of rich data. The twelve participants in this study shared their experiences of transitioning in serious illness, and by doing so, allowed this researcher to fill a gap in the literature. Findings from this study provide insight into how specific cultural factors influenced family transitions in serious illness. Furthermore, findings emphasize that factors came into play at different points along the illness continuum and had a unique significance for individual families. This is contrary to the assumption that generalizations can be made across populations, and re-emphasizes the need for patient-centered, family-focused, evidence-based care (IOM, 2015). 


\section{Limitations}

Four of the twelve participants in this study were males. Prior literature has identified barriers to male participation in bereavement studies that included feeling bad, uncomfortable, or experiencing pain when talking about grief (Skulason \& Helgason, 2010). Additionally, differences in in ways that males and females process emotional expression has been identified in the literature. Because of this, recruitment of males into bereavement studies is difficult (Guinther, Segal, Bogaards, 2003). It is unknown whether these were reasons that more males did not express interest in participation in this study. Future studies could be designed to include an equal number of male and female participants, or to focus solely on the experiences of either males or females.

In this study, additional sub-groups were also included. For example, four participants were health professionals. Because of their formal medical and/or nursing education, these participants had different knowledge and experiences that could influence views of transitions. Furthermore, six participants were primary caregivers while the remaining six participants played a more supportive role throughout illness. Primary caregivers may have a closer view of the illness transitions than family members acting in supportive roles. Further examination of or comparisons of sub-groups transitioning in illness is needed to fully understand the differences or similarities in how cultural factors influence transitions within each of the sub-groups.

In this study, retrospective interviews with participants occurred between 3 and 35 months of the death of the seriously ill person. In this sample, recollection of events did not seem to differ among those that experienced the death of a relative closer to or further away from the interview. The main difference observed among participants was that those who experienced the death of a relative closer to the time of the interview expressed more emotion 
throughout the interview than those who experienced the death of a relative further away from the time of the interview. Although participants readily recalled events, memory does have limitations regardless of time. To gain a better understanding of family transitions as they are experiencing them, prospective longitudinal studies are needed. Furthermore, to gain a family perspective that represents the entire family's view of transitions, interviews should be conducted with all family members. In this study, one or two family members represented each family.

\section{Summary}

The purpose of this qualitative descriptive study was to convey families' retrospective descriptions of the influence of cultural factors in the transition spanning from the point that a diagnosis was made up to the point when the family realized that the family members' disease or illness was progressive $\mathrm{n}$ nature, that it could be managed but not cured by medical treatment, and would ultimately result in death. Within this chapter, this researcher presented a summary of findings, related findings to relevant theoretical, conceptual, and empirical understandings of the topic. Additionally, within this chapter, implications for nursing practice, education, and research were presented. Finally, the author presented limitations and strengths of this study. Major findings from this study implicate that family/kinship, knowledge systems, community/social systems, and faith/religion influence family members' behaviors, understandings, and decisions at specific points throughout transitions in serious illness. Findings from this study indicate that transitions in serious illness are family-centric and families are involved very early in the illness, therefore nurses and other clinicians need to assess family involvement and include families in care early on and throughout the illness trajectory. Furthermore, analysis of data revealed that ways of knowing are diverse; families come to know 
about what to expect of illness transitions through a variety of ways. Nurses should assess families' baseline knowledge and be prepared to help families navigate the many ways that they come to know about the serious illness transitions. Caring behaviors and honest, forthright, patient-centered, family-focused communication methods are appreciated by families and were described as easing transitions. These skills should be taught in schools of nursing and reemphasized to practicing health care professionals. Finally, nurses and other clinicians must be aware that although all cultural factors were described as influencing family transitions in serious illness, there were individual variations in how factors influenced transitions. Careful assessment of the role of and importance of each cultural factor to family members transitioning in illness is needed before developing a plan of care. 


\section{References}

American Association of Colleges of Nursing. (2014). Essentials of Baccalaureate Education for Professional Nursing Practice. www.aacn.nche.edu/educationresources/BaccEssentials08.pdf

American Nurses Association. (2012). Care coordination and registered nurses' essential role. ANA position statement. Retrieved 2015, retrieved from http://nursingworld.org/MainMenuCategories/Policy-Advocacy/Positions-andResolutions/ANAPositionStatements/Position-Statements-Alphabetically/CareCoordination-and-Registered-Nurses-Essential-Role.html

American Psychological Association. (2014). Family caregiving. Retrieved, 2014, Retrieved from https://www.apa.org/about/gr/issues/cyf/caregiving-facts.aspx

Benzine, E., Olin, C., \& Persson, C. (2015). 'You put it all together' - families'evaluation of participating in family health conversations. Scandanavian Journal of Caring Sciences. 29; 136-144. Doi: $10.1111 / \mathrm{scs} .12141$

Blum, K., \& Sherman, D. W. (2010). Understanding the experience of caregivers: A focus on transitions. Seminars in Oncology Nursing, 26(4), 243-258. doi:10.1016/j.soncn.2010.08.005

Bourgeois \& Johnson (2004). Preparing for dying: Meaningful practices in palliative care. OMEGA, 49 (2), 99-107. 
Braun, U. K., Beyth, R. J., Ford, M. E., \& McCullough, L. B. (2008). Voices of African American, Caucasian, and Hispanic surrogates on the burdens of end-of-life decision making. JGIM: Journal of General Internal Medicine, 23(3), 267-274. doi:10.1007/s11606007-0487-7

Bullock, K. (2011). The influence of culture on end-of-life decision making. Journal of Social Work in End-of-Life \& Palliative Care, 7(1), 83-98. doi:10.1080/15524256.2011.548048

Carrion, I., V., \& Nedjat-Haiem, F. (2013). Caregiving for older Latinos at end-of-life: Perspectives from paid and family (unpaid) caregivers. American Journal of Hospice \& Palliative Medicine, 30(2), 183-191. doi:10.1177/1049909112448227

Cruz, E.V. \& Higginbottom, G. (2013). The use of focused ethnography in nursing research. Nurse Researcher, 20 (4), 36-43.

Csikai, E. L. (2006). Bereaved hospice caregivers' perceptions of the end-of-life care communication process and the involvement of health care professionals. Journal of Palliative Medicine, 9(6), 1300-1309. doi:10.1089/jpm.2006.9.1300

Csikia, E., \& Martin, S.,S. (2010). Bereaved hospice caregivers' views of the transition to hospice. Social Work in Health Care, 49(5), 387-400. doi:10.1080/00981380903426822

Dalgaard, K. M., Thorsell, G., \& Delmar, C. (2010). Identifying transitions in terminal illness trajectories: A critical factor in hospital-based palliative care. International Journal of Palliative Nursing, 16(2), 87-92. Retrieved from 
$\underline{\text { http://search.ebscohost.com/login.aspx?direct=true } \& d b=a 9 h \& A N=48990864 \& \text { site=ehost- }}$ $\underline{\text { live }}$

DeSanto-Madeya, S., Nilsson, M., Loggers, E. T., Paulk, E., Stieglitz, H., Kupersztoch, Y. M., \& Prigerson, H. G. (2009). Associations between United States acculturation and the end-oflife experience of caregivers of patients with advanced cancer. Journal of Palliative Medicine, 12(12), 1143-1149. doi:10.1089/jpm.2009.0063

Dolce, M. (2011). The internet as a source of health information: Experiences of cancer survivors and caregivers with healthcare providers. Oncology Nursing Forum, 38(3), 353359.

Donovan, R., Williams, A., Stajduhar, K., Brazil, K., \& Marshall, D. (2011). The influence of culture on home-based family caregiving at end-of-life: A case study of Dutch reformed family care givers in ontario, canada. Social Science \& Medicine, 72(3), 338-346. doi:10.1016/j.socscimed.2010.10.010

Dosser, I., \& Kennedy, C. (2012). Family carers' experiences of support at the end of life: Carers' and health professionals' views. International Journal of Palliative Nursing, 18(10), 491497. Retrieved from http://search.ebscohost.com/login.aspx?direct=true \&db=a9h\&AN=83815779\&site=ehost$\underline{\text { live }}$

Dubenske, L. L., Wen, K. Y., Gustafson, D. H., Guarnaccia, C. A., Cleary, J. F., Dinauer, S. K., \& McTavish, F. M. (2008). Caregivers' differing needs across key experiences of the advanced cancer disease trajectory. Palliative \& Supportive Care, 6(3), 265-272. Retrieved 
from

http://search.ebscohost.com/login.aspx?direct=true \&db=c8h\&AN=2009998041\&site=ehost$\underline{\text { live }}$

Duggleby, W., \& Berry, P. (2005). Transitions and shifting goals of care for palliative patients and their families. Clinical Journal of Oncology Nursing, 9(4), 425-428. doi:10.1188/05.CJON.425-428

Edwards, S., B., Olson, K., Koop, P., M., \& Northcott, H., C. (2012). Patient and family caregiver decision making in the context of advanced cancer. Cancer Nursing, 35(3), 178186. doi:10.1097/ncc.0b013e31822786f6

Elo, S., \& Kyngäs, H. (2008). The qualitative content analysis process. Journal of Advanced Nursing, 62(1), 107-115. doi:10.1111/j.1365-2648.2007.04569.x

Ford, D. W., Nietert, P. J., Zapka, J., Zoller, J. S., \& Silvestri, G. A. (2008). Barriers to hospice enrollment among lung cancer patients: A survey of family members and physicians. Palliative \& Supportive Care, 6(4), 357-362. Retrieved from http://search.ebscohost.com/login.aspx?direct=true \&db=c8h\&AN=2010102938\&site=ehost$\underline{\text { live }}$

Fox, S., \& Jones, S. (2009). The social life of internet users. Washington, DC: Pew Internet \& American Life Project.

Gallo, V. (2013). Observations of families transitioning to hospice. Unpublished pilot study, Department of Nursing, West Virginia University, Morgantown, WV. 
Gerard, J. (2011). Health sciences literature review made easy: The matrix method. Jones and Bartlett. Sudbury, MA.

Giesbrecht, M., Crooks, V. A., Williams, A., \& Hankivsky, O. (2012). Critically examining diversity in end-of-life family caregiving: Implications for equitable caregiver support and Canada's compassionate care benefit. International Journal for Equity in Health, 11(1), 6577. doi:10.1186/1475-9276-11-65

Guinther, P., Segal, D., Bogaards, J. (2003). Gender differences in emotional processing among bereaved older adults. Journal of Loss and Trauma, 8, 15-33. doi: $10.1080 / 15325020390168708$

Gunaratnam, Y. (2007). Intercultural palliative care: Do we need cultural competence? International Journal of Palliative Nursing, 13(10), 470-477. Retrieved from http://search.ebscohost.com/login.aspx?direct=true \&db=a9h\&AN=27584235\&site=ehost$\underline{\text { live }}$

Hanson, S. (2005). Introduction to family health care nursing. In S.M.H. Hanson, V. GedalyDuff \& J.R. Kaakinen (Eds), Family health care nursing: Theory, practice and research $\left(3^{\text {rd }}\right.$ ed., pp 3-38). Philadelphia, F.A.Davis.

Herbert, R. S., \& Schulz, R. (2006). Caregiving at the end of life. Journal of Palliative Medicine, 9(5), 1174-1187. doi:10.1089/jpm.2006.9.1174

Hsieh, H., \& Shannon, S. (2005). Three approaches to qualitative content analysis. Qualitative Health Research, 15(9), 1277-1288. doi:10.1177/1049732305276687 
Hui, D., Nooruddin, Z., Didwaniya, N., Dev, R., Cruz, D. L., Kim, S. H., . . Bruera, E. (2014). Concepts and definitions for "Actively dying," "End of life," "Terminally ill," "Terminal care," and "Transition of care": A systematic review. Journal of Pain \& Symptom Management, 47(1), 77-89. doi:10.1016/j.jpainsymman.2013.02.021

Hurley, K. (2014). To fight or not to fight: A cancer psychotherapist with cancer confronts the battle metaphor. Women and Therapy. 37(3/4) 311.

Imber-Black, E. (2014). Will talking about it make it worse? Family conversations in the context of chronic and life-shortening illness. Journal of Family Nursing, 21(2), 151-163.

Institute of Medicine (IOM). (2015). Dying in America: Improving quality and honoring individual preferences near the end of life. The National Academies Press. Washington, D.C.

Kelley, A. S., Wegner, N. S., \& Sarkisian, C. A. (2010). Opiniones: End-of-life care preferences and planning of older Latinos. Journal of the American Geriatrics Society, 58(6), 11091116. doi:10.1111/j.1532-5415.2010.02853.x

Koffman, J., Higginson, I. J., Hall, S., Riley, J., McCrone, P., \& Gomes, B. (2012). Bereaved relatives' views about participating in cancer research. Palliative Medicine, 26(4), 379-383. doi:10.1177/0269216311405091

Kralik, D., Visentin, K., \& Van Loon, A. (2006). Transition: A literature review. Journal of Advanced Nursing, 55(3), 320-329. doi:10.1111/j.1365-2648.2006.03899.x 
Kramer, B. J., \& Auer, C. (2005). Challenges to providing end-of-life care to low-income elders with advanced chronic disease: Lessons learned from a model program. Gerontologist, 45(5), 651-660. Retrieved from http://search.ebscohost.com/login.aspx?direct=true \&db=a9h\&AN=18458405\&site=ehost$\underline{\text { live }}$

Kreling, B., Selsky, C., Perret-Gentil, M., Huerta, E. E., \& Mandelblatt, J. S. (2010). 'The worst thing about hospice is that they talk about death': Contrasting hospice decisions and experience among immigrant central and south American Latinos with US-born white, nonLatino cancer caregivers Sage Publications, Ltd. doi:10.1177/0269216310366605

Lawson, B., Burge, F. I., Critchley, P., \& McIntyre, P. (2006). Factors associated with multiple transitions in care during the end-of-life following enrollment in a comprehensive palliative care program. BMC Palliative Care, 5, 4-10. doi:10.1186/1472-684X-5-4

Leininger, M. (1967). The culture concept and its relevance to nursing. The Journal of Nursing Education, 6(2), 27--37.

Leininger, M. (1988). Leininger's theory of nursing: Cultural care diversity and universality. Nursing Science Quarterly, 1(4), 152--160.

Lockie, S. J., Bottorff, J. L., Robinson, C. A., \& Pesut, B. (2010). Experiences of rural family caregivers who assist with commuting for palliative care. CJNR: Canadian Journal of Nursing Research, 42(1), 74-91. Retrieved from http://search.ebscohost.com/login.aspx?direct=true \&db=psyh\&AN=2010-06522004\&site $=$ ehost-live 
Meeker, M., \& Jezewski, M. (2005). Family decision making at end-of-life. Palliative and Supportive Care, 3, 131--142. doi:10.1017/s1478951505050212

Meleis, A. (2010). Transitions theory: middle-range and situation-specific theories in nursing research and practice. Springer Publishing Company, NY.

Meleis, A., Sawyer, L., Im, E., Hilfinger, D., \& Schumacher, K. (2000). Experiencing transitions: An emerging middle-range theory. Advances in Nursing Science, 23(1), 12-28.

National Hospice and Palliative Care Organization. (2013). NHPCO's facts and figures: Hospice care in America. .National Hospice and Palliative Care Organization. Retrieved from http://www.nhpco.org/hospice-statistics-research-press-room/facts-hospice-and-palliativecare

Northfield, S., \& Nebauer, M. (2010). The caregiving journey for family members of relatives with cancer. Clinical Journal of Oncology Nursing, 14(5), 567-577. Retrieved from http://search.ebscohost.com/login.aspx?direct=true $\&$ db=a9h\&AN=54013836\&site=ehost$\underline{\text { live }}$

Paul, F., \& Rattray, J. (2008). Short- and long-term impact of critical illness on relatives: Literature review. Journal of Advanced Nursing, 62(3), 276-292. doi:10.1111/j.13652648.2007.04568.x

Penman, J., Oliver, M., \& Harrington, A. (2013). The relational model of spiritual engagement depicted by palliative care clients and caregivers. International Journal of Nursing Practice, 19(1), 39-46. doi:10.1111/ijn.12035 
Penrod, J., Hupcey, J. E., Shipley, P. Z., Loeb, S. J., \& Baney, B. (2012). A model of caregiving through the end of life: Seeking normal. Western Journal of Nursing Research 34 (2), 174193. doi:10.1177/0193945911400920

Phillips, L. R., \& Reed, P. G. (2009). Into the abyss of someone else's dying the voice of the endof-life caregiver. Clinical Nursing Research, 18(1), 80-97. Retrieved from http://search.ebscohost.com/login.aspx?direct=true \&db=a9h\&AN=36617138\&site=ehost$\underline{\text { live }}$

Phillips, L. R., \& Reed, P. G. (2010). End-of-life Caregiver's perspectives on their role: Generative caregiving. Gerontologist, 50(2), 204-214. Retrieved from http://search.ebscohost.com/login.aspx?direct=true \&db=a9h\&AN=50641009\&site=ehost$\underline{\text { live }}$

Pinnock, H., Kendall, M., Murray, S. A., Worth, A., Levack, P., Porter, M., . . Sheikh, A. (2011). Living and dying with severe chronic obstructive pulmonary disease: Multiperspective longitudinal qualitative study. BMJ: British Medical Journal, 342(7791), 1-10. Retrieved from http://search.ebscohost.com/login.aspx?direct=true \&db=psyh\&AN=2011$\underline{02295-001 \& \text { site }=\text { ehost-live }}$

Polit, D., \& Beck, C. (Eds.). (2014). Essentials of nursing research; appraising evidence for nursing practice (8th ed.). Philadelphia, PA: Wolters Kluwer Health/Lippincott Williams \& Wilkins. 
Powers, B. A., Norton, S. A., Schmitt, M. H., Quill, T. E., \& Metzger, M. (2011). Meaning and practice of palliative care for hospitalized older adults with life limiting illnesses. Journal of Aging Research, 1-8. doi:10.4061/2011/406164

Rainey, H., Ehrich, K., Mackintosh, N., Dip, M. \& Sandall, J. (2013). The role of patients and their relatives in 'speaking up' about their own safety - a qualitative study of acute illness. Health Expectations, 18, 392-405. doi: 10.111/hex.12044

Reinke, L. F., Engelberg, R. A., Shannon, S. E., Wenrich, M. D., Vig, E. K., Back, A. L., \& Curtis, J. R. (2008). Transitions regarding palliative and end-of-life care in severe chronic obstructive pulmonary disease or advanced cancer: Themes identified by patients, families, and clinicians. Journal of Palliative Medicine, 11(4), 601-609. doi:10.1089/jpm.2007.0236

Rosenblatt, P. C. (1995). Ethics of qualitative interviewing with grieving families. Death Studies, 19(2), 139. Retrieved from http://search.ebscohost.com/login.aspx?direct=true\&db=a9h\&AN=9512294071\&site=ehost$\underline{\text { live }}$

Russ, A., \& Kaufman, S. (2005). Family perceptions of prognosis, silence, and the "Suddenness" of death. Culture, Medicine \& Psychiatry, 29(1), 103-123. doi:10.1007/s11013-005-4625-6

Ruthin, J., Holfeld, B., \& Hanson, G. (2012). The role of positive thinking in social perceptions of cancer outcomes. Psychological Health, 27(10), 1244-1258.

Skulason, B. \& Helgason, A. (2010). Identifying obstacles to participation in a questionnaire survey on widowers' grief. BMC Palliative Care, 9 (7). doi: 10.1186/1472-684X-9-7 
Sandelowski, M. (2000). Focus on research methods: Whatever happened to qualitative description? Research in Nursing \& Health, 23(4), 334-340. Retrieved from http://search.ebscohost.com/login.aspx?direct=true $\& \mathrm{db}=\mathrm{c} 8 \mathrm{~h} \& \mathrm{AN}=2000072570 \&$ site=ehost$\underline{\text { live }}$

Sandelowski, M. (2010). What's in a name? Qualitative description revisited. Research in Nursing \& Health, 33(1), 77-84. doi:10.1002/nur.20362

Sanders, J. (2015). Finding the right words at the right time - high-value advance care planning. New England Journal of Medicine. 372(7), 598-599.

Spichiger, E. (2008). Living with terminal illness: Patient and family experiences of hospital end-of-life care. International Journal of Palliative Nursing, 14(5), 220-228. Retrieved from http://search.ebscohost.com/login.aspx?direct=true $\& d b=a 9 h \& A N=32759160 \&$ site=ehost$\underline{\text { live }}$

Stroebe, M., Stroebe, W., \& Schut, H. (2003). Bereavement research: Methodological issues and ethical concerns. Palliative Medicine, 17(3), 235-240. doi:10.1191/0269216303pm768rr

Tallman, K., Greenwald, R., Reidenouer, A., \& Pantel, L. (2012). Living with advanced illness: Longitudinal study of patient, family, and caregiver needs. The Permanente Journal, 16(3), 28-35.

Tavakol, M., \& Zeinaloo, A. (2004). Medical research paradigms: Positivistic inquiry paradigm versus naturalistic inquiry paradigm. Journal of Medical Education, 5(2), 75-80. 
The Joint Commission. (2010). Advancing effective communication, cultural competence, and patient-and family-centered care: A roadmap for hospitals. Oakbrook Terrace, IL: The Joint Commission. Retrieved from http://www.jointcommission.org/assets/1/6/aroadmapforhospitalsfinalversion727.pdf

U.S. Census Bureau. (2012). Statistical Abstract of the United States. Retrieved from http://www.census.gov/compendia/statab/2012/tables/12s0105.pdf

Vandenberg, H. E. R. (2010). Culture theorizing past and present: Trends and challenges. Nursing Philosophy, 11(4), 238-249. doi:10.1111/j.1466-769X.2010.00453.x

Waldrop, D. P., Kramer, B. J., Skretny, J. A., Milch, R. A., \& Finn, W. (2005). Final transitions: Family caregiving at the end of life. Journal of Palliative Medicine, 8(3), 623-638. doi:10.1089/jpm.2005.8.623

West Virginia Department of Health and Human Services (WVDHHR). (2010). West Virginia Vital Statistics, sixty-fourth annual report. Retrieved from http://www.wvdhhr.org/bph/hsc/pubs/vital/2010/2010Vital.pdf

West Virginia University (2015) Human Research Protections Progam Office of Research Integrity and Compliance. Standard operating procedures. Taken from http://oric.research.wvu.edu/files/d/37c9edba-14cd-44dd-a24c396c1698efdd/IRB\%20Policies\%202015.02.11.pdf

Whitehead, T. (2004). What is ethnography? Methodological, ontological, and epistemological attributes. Ethnographically informed community and cultural assessment research systems 
working paper series. Retrieved on March 1, 2014 from

http://www.cusag.umd.edu/documents/WorkingPapers/EpiOntAttrib.pdf

Wittenberg-Lyles, E., Goldsmith, J., Demiris, G., Oliver, D., Parker, \& Stone, J. (2012). The impact of family communication patterns on hospice family caregivers: A new typology. Journal of Hospice \& Palliative Nursing, 14(1), 25-33.

doi:10.1097/NJH.0b013e318233114b

World Health Organization (2007). People-centered health care: A policy framework. World Health Organization. Retrieved from http://www.wpro.who.int/health_services/people_at_the_centre_of_care/documents/ENGPCIPolicyFramework.pdf

World Health Organization. (2014). Palliative care is an essential part of cancer control. Retrieved, 2014, Retrieved from http://www.who.int/cancer/palliative/en/

Wright, A., Stieglitz, H., Kupersztoch, Y, Paulk, M., Kim, Y., Katz, I.,, . . Prigerson, H. G. (2013). United States acculturation and cancer patients' end-of-life care. Plos One, 8(3), 110. doi:10.1371/journal.pone. 0058663

Zeytinoglu, M. (2011). Talking it out: Helping our patients live better while dying. Annals of Internal Medicine. 154(12), 830-W300 
Appendix 
Appendix 2.1: Literature Review Matrix with author, population, stage of illness, purpose and findings.

\begin{tabular}{|c|c|c|c|c|}
\hline Author, year & Population & Stage & Purpose & Findings \\
\hline $\begin{array}{l}\text { Bourgeois \& } \\
\text { Johnson }(2003)\end{array}$ & $\begin{array}{l}\text { Family of } \\
\text { dying } \\
\text { persons }\end{array}$ & EOL & $\begin{array}{l}\text { Use family stories as exemplars to } \\
\text { illustrate how culture shapes attitudes } \\
\text { toward dying and practices adopted by } \\
\text { groups that assist them to make sense of } \\
\text { their world. }\end{array}$ & $\begin{array}{l}\text { Family EOL rituals facilitated coping, diminished fear and } \\
\text { grief r/t end-of-life. }\end{array}$ \\
\hline Bullock (2011) & $\begin{array}{l}\text { African } \\
\text { Americans } \\
\text { and } \\
\text { Caucasians }\end{array}$ & EOL & $\begin{array}{l}\text { Explore values, norms, attitudes and } \\
\text { behaviors surrounding EOL care } \\
\text { decisions in African Americans }\end{array}$ & $\begin{array}{l}\text { A higher percentage of Caucasians completed Ad. } \\
\text { Directives than African American } \\
\text { AA relied on family and faith to intervene in EOL care } \\
\text { decisions, less trustful of HCP. } \\
\text { AA distrustful of Advanced Directives. AA distrustful of } \\
\text { hospice. } \\
\text { Caucasians more confident in HC team to provide EOL } \\
\text { care. Positive views of hospice. Viewed religion as a } \\
\text { support system. }\end{array}$ \\
\hline $\begin{array}{l}\text { Carrion \& } \\
\text { Nedjat-Haiem, } \\
(2013)\end{array}$ & $\begin{array}{l}\text { Latino } \\
\text { Family } \\
\text { Caregivers }\end{array}$ & Terminal $*$ & $\begin{array}{l}\text { Explore settings in which Latino } \\
\text { caregivers provide care and the } \\
\text { challenges they face when caring for } \\
\text { terminally ill Latinos. }\end{array}$ & $\begin{array}{l}\text { Challenges identified during caregiving experience: } \\
\text { language barriers, work/school/care provision balance, no } \\
\text { family lived close by to help (lived in native country) }\end{array}$ \\
\hline $\begin{array}{l}\text { Csikia \& Martin } \\
(2010)\end{array}$ & $\begin{array}{l}\text { Bereaved } \\
\text { hospice } \\
\text { caregivers }\end{array}$ & $\begin{array}{l}\text { Hospice } \\
\text { EOL }\end{array}$ & $\begin{array}{l}\text { Explore bereaved hospice caregivers } \\
\text { experiences regarding communication } \\
\text { with various HCP about patient's serious } \\
\text { illness and EOL care options. }\end{array}$ & $\begin{array}{l}\text { Families valued involvement of patient or acknowledgement } \\
\text { of patient wishes in discussions about care, disclosure or } \\
\text { prognosis from HCP (not always given), wanted to know } \\
\text { about hospice services sooner }\end{array}$ \\
\hline $\begin{array}{l}\text { Dalgaard, } \\
\text { Thorsell, Delmar } \\
(2010)\end{array}$ & $\begin{array}{l}\text { Patients with } \\
\text { incurable } \\
\text { hematologic } \\
\text { disease }\end{array}$ & $\begin{array}{l}\text { Palliative } \\
\text { care* }\end{array}$ & $\begin{array}{l}\text { Describe significance of identification } \\
\text { and explicit communication of transitions } \\
\text { in incurable illness trajectories. }\end{array}$ & $\begin{array}{l}\text { Family acceptance of prognosis was listed as a barrier to } \\
\text { transitioning to late palliative phase. } \\
\text { Physicians often discussed prognosis and palliative care } \\
\text { options very late in the illness trajectory. } \\
\text { Health care providers focus very much on the nature of the } \\
\text { disease when holding discussions with patients and families. } \\
\text { Earlier communication of care options gave families a new } \\
\text { sense of hope and meaning. }\end{array}$ \\
\hline $\begin{array}{l}\text { DeSanto-Madeya } \\
\text { et al. (2009) }\end{array}$ & $\begin{array}{l}\text { Caucasian } \\
\text { and Hispanic } \\
\text { Patients with } \\
\text { advanced } \\
\text { cancer and }\end{array}$ & Terminal* & $\begin{array}{l}\text { Examine extent to which treatment } \\
\text { factors (preferences, physician care-giver } \\
\text { communication) and psychosocial factors } \\
\text { (mental health, additional services, } \\
\text { internal/external support) differ based on }\end{array}$ & $\begin{array}{l}\text { Acculturation scores higher in white and black and lower in } \\
\text { Hispanic participants. } \\
\text { High scores on the acculturation scale signified that the } \\
\text { participant claimed to be more Americanized than tied to } \\
\text { their racial heritage. }\end{array}$ \\
\hline
\end{tabular}




\begin{tabular}{|c|c|c|c|c|}
\hline & $\begin{array}{l}\text { primary } \\
\text { caregivers }\end{array}$ & & $\begin{array}{l}\text { the acculturation of the caregivers of } \\
\text { patients with advanced cancer. }\end{array}$ & $\begin{array}{l}\text { Higher acculturation scores associated with use of mental } \\
\text { health services, desire for additional support services. } \\
\text { Lower acculturation scores associated with spiritual support } \\
\text { from religious communities, stronger family ties, and higher } \\
\text { levels of self-efficacy. }\end{array}$ \\
\hline $\begin{array}{l}\text { Donovan et al. } \\
\text { (2010) }\end{array}$ & $\begin{array}{l}\text { Family } \\
\text { caregiver }\end{array}$ & $\begin{array}{l}\text { Palliative } \\
\text { Care Phase* }\end{array}$ & $\begin{array}{l}\text { Determine what factors are significant to } \\
\text { Dutch reformed family caregivers as a } \\
\text { cultural entity in the provision of end of } \\
\text { life care. }\end{array}$ & $\begin{array}{l}\text { Cultural attitudes towards care described: } \\
\text { Caregiving is a family responsibility } \\
\text { Caregiver role associated with relationship to family } \\
\text { member } \\
\text { Home care and hospice valued by caregivers } \\
\text { Religious beliefs and coping strategies } \\
\text { Religion facilitated coping with illness and experiences } \\
\text { Belief in God increased confidence in decisions } \\
\text { Faith challenged when illness became overwhelming or } \\
\text { death imminent } \\
\text { Church community was a source of emotional and physical } \\
\text { support for caregivers } \\
\text { Culturally informed care-seeking behaviors } \\
\text { Medical services valued } \\
\text { Conferred with medical professional regarding care } \\
\text { Futile treatments decreased and QOL measures undertaken } \\
\text { due to faith and religious beliefs in an afterlife } \\
\text { Self-reliance and independence valued - reluctant to accept } \\
\text { outside help } \\
\text { Pride of ill person led to downplay of symptoms and } \\
\text { sometimes resulted in not getting needed care }\end{array}$ \\
\hline $\begin{array}{l}\text { Duggleby et al. } \\
(2010)\end{array}$ & $\begin{array}{l}\text { Rural cancer } \\
\text { patients and } \\
\text { their family } \\
\text { caregivers. }\end{array}$ & $\begin{array}{l}\text { Palliative } \\
\text { care* }\end{array}$ & $\begin{array}{l}\text { Explore transitions experience of older } \\
\text { adults and family receiving palliative } \\
\text { care. Develop a theory of transition in } \\
\text { population. }\end{array}$ & $\begin{array}{l}\text { Transitions experienced by patients and families included: } \\
\text { including environmental changes, relationship and role } \\
\text { changes, physical and mental health changes, changes to } \\
\text { activities of daily living. } \\
\text { Positive adaptations to transitions were impacted by timely } \\
\text { communication, provision of essential information, and the } \\
\text { presence of family and community support networks. } \\
\text { Value of individuality and community and lack of } \\
\text { accessibility to healthcare were identified themes related to } \\
\text { rural patients. }\end{array}$ \\
\hline $\begin{array}{l}\text { Edwards, Olson, } \\
\text { Koop \& } \\
\text { Northcott (2012) }\end{array}$ & $\begin{array}{l}\text { Patients with } \\
\text { advanced } \\
\text { cancer and }\end{array}$ & $\begin{array}{l}\text { Across } \\
\text { illness } \\
\text { trajectory }\end{array}$ & $\begin{array}{l}\text { Understand decision-making process that } \\
\text { occurs between dying individual and his } \\
\text { or her family caregiver. }\end{array}$ & $\begin{array}{l}\text { Family caregivers satisfied when death was managed in a } \\
\text { way consistent with patient's wishes. Two people had to be } \\
\text { willingly and actively engaged with each other. }\end{array}$ \\
\hline
\end{tabular}




\begin{tabular}{|c|c|c|c|c|}
\hline & $\begin{array}{l}\text { family } \\
\text { caregivers }\end{array}$ & & & $\begin{array}{l}\text { Basic social process had five stages; (1). Vague symptoms, } \\
\text { receipt of diagnosis (2). Seeking treatment and cure; } \\
\text { (3). Preparing for death of loved ending with death of loved } \\
\text { one; (4). After death (5). Moving on after death. The } \\
\text { treatment phase was sometimes hindered by barriers within } \\
\text { the healthcare system. Referrals had to be fought for, } \\
\text { alternative treatments explored. Additionally, during this } \\
\text { time, family caregivers had to modify their life and work } \\
\text { activities to help their family one get necessary treatment } \\
\text { and care. }\end{array}$ \\
\hline $\begin{array}{l}\text { Giesbercht, } \\
\text { Crooks, } \\
\text { Williams, \& } \\
\text { Hankivsky }\end{array}$ & $\begin{array}{l}\text { Formal } \\
\text { palliative } \\
\text { caregivers in } \\
\text { Canada }\end{array}$ & EOL & $\begin{array}{l}\text { Understand the implications of caregiver } \\
\text { diversity for the CCB }\end{array}$ & $\begin{array}{l}\text { 5 factors emerged as differences among end-of-life family } \\
\text { caregivers that influence their experience as a caregiver. } \\
\text { 1. Culture; clash of personal beliefs with Canadian } \\
\text { HC system - differing priorities and needs. } \\
\text { Language barriers contributed to challenges with } \\
\text { family caregiving and determination of caregiver } \\
\text { needs. } \\
\text { 2. Gender: role of caregiver ascribed mostly to } \\
\text { women. } \\
\text { 3. Geography: Where caregiver and patient live } \\
\text { determine access to services. Isolated, low } \\
\text { populated areas created challenges for family } \\
\text { caregivers to access end of life support services. In } \\
\text { areas where young people migrate out of, families } \\
\text { have less supportive persons around them. Rural } \\
\text { areas less access to care or longer commutes to } \\
\text { care. } \\
\text { 4. Life-course stage: elderly caring for spouses - } \\
\text { more time to care because of retirement, less } \\
\text { physical ability to care because of own health } \\
\text { conditions or diminished physical abilities. } \\
\text { Children caring for older parent had dual } \\
\text { responsibilities to their own spouses and children } \\
\text { as well as to dying parent. } \\
\text { Material resources: Related to access to resources } \\
\text { (income, equipment, medication, respite and home } \\
\text { care) }\end{array}$ \\
\hline $\begin{array}{l}\text { Kelley, Wegner, } \\
\text { \& Sarkisian } \\
(2010)\end{array}$ & $\begin{array}{l}\text { Elderly } \\
\text { caregivers }\end{array}$ & EOL & $\begin{array}{l}\text { Measure EOL care preferences and } \\
\text { advanced care planning as well as } \\
\text { attitudes towards three culture based }\end{array}$ & $\begin{array}{l}\text { Completion of advanced directives was more likely in } \\
\text { participants: with higher income and more education, those }\end{array}$ \\
\hline
\end{tabular}




\begin{tabular}{|c|c|c|c|c|}
\hline & & & $\begin{array}{l}\text { constructs: family-centered decision- } \\
\text { making, patient autonomy, trust in } \\
\text { healthcare providers. Also included } \\
\text { demographics. }\end{array}$ & $\begin{array}{l}\text { having experienced a family member dying in the hospital, } \\
\text { English speaking, with higher acculturation, born in the US. } \\
\text { Completion of advanced directives was less likely in } \\
\text { participants: who rated health as fair or poor, preferred } \\
\text { limited autonomy. } \\
\text { If unable to make medical decisions, } 64 \% \text { of participants } \\
\text { favored family-centered decision making rather than a single } \\
\text { surrogate decision maker. }\end{array}$ \\
\hline $\begin{array}{l}\text { Kreling et al., } \\
2010\end{array}$ & $\begin{array}{l}\text { Latino- } \\
\text { American } \\
\text { caregivers }\end{array}$ & Hospice & $\begin{array}{l}\text { Describe experiences of Latino-American } \\
\text { cancer caregivers who enrolled in hospice } \\
\text { and evaluate whether culture influence } \\
\text { experiences }\end{array}$ & $\begin{array}{l}\text { Decision making and caregiving experiences between the } \\
\text { two groups varied; Latino cultural values of denial, secrecy } \\
\text { about prognosis and collective, family-centered system } \\
\text { influenced hospice decisions. When compared with their } \\
\text { white, non-Latino counterparts, Latinos: had no knowledge } \\
\text { or misconceptions about hospice, were distrustful of hospice } \\
\text { services, decided enroll in hospice during a crisis } \\
\text { hospitalization, had concerns over ability to pay for services, } \\
\text { were not fully accepting of terminal prognosis of patient, } \\
\text { avoided discussions of death - even w/I family - family in } \\
\text { charge, wanted to make decisions without involving the } \\
\text { patient. }\end{array}$ \\
\hline $\begin{array}{l}\text { Lockie et al., } \\
\text { (2010) }\end{array}$ & $\begin{array}{l}\text { Rural Family } \\
\text { Palliative } \\
\text { Caregivers }\end{array}$ & $\begin{array}{l}\text { Palliative } \\
\text { Care* }\end{array}$ & $\begin{array}{l}\text { Investigate the experiences of rural family } \\
\text { palliative caregivers who supported } \\
\text { advanced cancer patients receiving } \\
\text { palliative treatment at a regional cancer } \\
\text { center. }\end{array}$ & $\begin{array}{l}\text { Family palliative caregivers had multiple responsibilities in } \\
\text { addition to transporting ill person. } \\
\text { Community support networks were important to family } \\
\text { caregivers. } \\
\text { Family palliative caregivers were dedicated to help ill } \\
\text { person; tried to make the best of it. } \\
\text { Long distance from center, poor road conditions, and } \\
\text { vehicle problems were barriers to transporting. }\end{array}$ \\
\hline $\begin{array}{l}\text { Penman, Oliver } \\
\text { \& Harrington } \\
\text { (2013) }\end{array}$ & $\begin{array}{l}\text { Patients and } \\
\text { caregivers } \\
\text { enrolled in } \\
\text { palliative } \\
\text { care services }\end{array}$ & $\begin{array}{l}\text { Palliative } \\
\text { care* }\end{array}$ & $\begin{array}{l}\text { Describe the essence of spiritual } \\
\text { engagement through lived experiences of } \\
\text { palliative care clients and caregivers. }\end{array}$ & $\begin{array}{l}\text { Spirituality helped cope with disease and transformed roles } \\
\text { of caregiver - promoted strength in caregivers. } \\
\text { Engaging in spirituality was equated with demonstrations of } \\
\text { love, compassion and altruism. } \\
\text { Expressions of love included engaging in religious practices } \\
\text { such a praying and participating in normative cultural } \\
\text { behaviors, such as sharing a meal. }\end{array}$ \\
\hline $\begin{array}{l}\text { Penrod et al. } \\
\text { (2012) }\end{array}$ & $\begin{array}{l}\text { EOL } \\
\text { caregivers }\end{array}$ & $\begin{array}{l}\text { Across } \\
\text { illness } \\
\text { trajectory }\end{array}$ & $\begin{array}{l}\text { Explicate a theoretical model of the } \\
\text { phases of transitions of EOL caregiving } \\
\text { across complex EOL disease trajectories } \\
\text { - pre-diagnosis through bereavement. }\end{array}$ & $\begin{array}{l}\text { Model of caregiving through EOL - "seeking normal" } \\
\text { Disruptions along illness trajectory were followed by quest } \\
\text { for normal. Families processed information to create a } \\
\text { pattern among chaos. }\end{array}$ \\
\hline
\end{tabular}




\begin{tabular}{|c|c|c|c|c|}
\hline & & & & $\begin{array}{l}\text { Interpretation of events by caregivers was key to successful } \\
\text { management of complex role demands. Family members } \\
\text { often experienced shock at sharp physical and cognitive } \\
\text { changes to ill person. } \\
\text { Four phases of caregiving identified. Transitions between } \\
\text { phases smoothed by understanding of events by caregivers. } \\
\text { Course of phases varied. Critical shaping factor was } \\
\text { certainty of death associated with specific disease of ill } \\
\text { family member. Some diseases are typified by an abrupt } \\
\text { shift in care (cure to comfort) such as HIV and Cancer; } \\
\text { others typified by progressive deterioration and anticipated } \\
\text { death (ALS); and others followed an unexpected death } \\
\text { trajectory, where acknowledgement of certainty of death } \\
\text { came late (CHF). } \\
\text { 1. Sensing a disruption - began when change in } \\
\text { family members health realized and ended when } \\
\text { diagnosis of life-limiting illness was received. } \\
\text { 2. Challenging normal; began when caregiver and } \\
\text { recipient joined forces to seek cure or remission of } \\
\text { disease. Doctors orders followed, attempt at } \\
\text { maintaining normal life. Ended when patient and } \\
\text { family were out of options to combat illness, } \\
\text { terminality of illness acknowledged. } \\
\text { Building a new normal; began when terminality } \\
\text { was acknowledged, family member caregiving } \\
\text { responsibilities increased over time, fulfilling } \\
\text { previous normal activities and roles difficult. } \\
\text { Ended at death of family member. } \\
\text { 4. Reinventing normal. Began after death of loved } \\
\text { one. Caregiver sought to find a new life, take on } \\
\text { new roles, and cherish memories. Some caregivers } \\
\text { became stuck in role as caregiver. }\end{array}$ \\
\hline Phillips (2009) & $\begin{array}{l}\text { Family } \\
\text { caregiver }\end{array}$ & EOL & $\begin{array}{l}\text { Understand family caregiver's } \\
\text { perspectives of providing care to elders } \\
\text { facing expected death from life limiting } \\
\text { chronic illness }\end{array}$ & $\begin{array}{l}\text { Uncertainty experienced by caregivers. Caregivers claimed } \\
\text { that the experience was often overwhelming. } \\
\text { Responsibilities and relationships were complex, navigating } \\
\text { medical system was difficult and conflict among family } \\
\text { members was experienced. } \\
\text { Family caregivers feared doing harm to ill elder; } \\
\text { unanticipated episodes of symptoms evoked fear among } \\
\text { family caregivers. }\end{array}$ \\
\hline
\end{tabular}




\begin{tabular}{|c|c|c|c|c|}
\hline & & & & $\begin{array}{l}\text { Caregivers anguished over patient suffering; felt helpless } \\
\text { and feelings of helplessness lasted beyond death. } \\
\text { Caregiving experience was meaningful - allowed them to } \\
\text { experience closeness with family member, participate in } \\
\text { special moments with family member, and witness miracles. } \\
\text { Changes to relationship norms were experienced by } \\
\text { caregivers; for example, children had to become caretakers } \\
\text { for parents. }\end{array}$ \\
\hline $\begin{array}{l}\text { Pinnock, et al. } \\
\text { (2011) }\end{array}$ & $\begin{array}{l}\text { COPD } \\
\text { patients and } \\
\text { caregivers }\end{array}$ & EOL & $\begin{array}{l}\text { To understand the EOL needs of persons } \\
\text { with COPD and informal and professional } \\
\text { carers }\end{array}$ & $\begin{array}{l}\text { Fatalistic attitude of patients and informal carers "COPD } \\
\text { was a way of life, just the way it is." } \\
\text { Transitions experienced included times of "wellness" when } \\
\text { exacerbations were not experienced. } \\
\text { Patient and family surprised at introduction of hospice or } \\
\text { palliative services. }\end{array}$ \\
\hline $\begin{array}{l}\text { Powers et al., } \\
2011\end{array}$ & $\begin{array}{l}\text { Seriously ill } \\
\text { older adults } \\
\text { and their } \\
\text { families }\end{array}$ & $\begin{array}{l}\text { Palliative } \\
\text { care and } \\
\text { EOL* }\end{array}$ & $\begin{array}{l}\text { Illustrate distinctions and intersections of } \\
\text { palliative care and end of life services of } \\
\text { older adults who have received acute care } \\
\text { hospital palliative care consultation } \\
\text { services }\end{array}$ & $\begin{array}{l}\text { 4 practice paradigms described } \\
\text { 1. End of life transitions (turning to palliative care): } \\
\text { certainty of impending death, uncertainty about } \\
\text { when death will occur and QOL of ill person until } \\
\text { then - family desired information that was } \\
\text { individualized to their needs "not too much, not too } \\
\text { little." At a time when they were ready to receive } \\
\text { it. Confusion and conflict experienced among } \\
\text { family and patient when they received opposing or } \\
\text { conflicting information from care providers. } \\
\text { 2. Prognostic Uncertainty: probable future outcomes } \\
\text { of patients' conditions cannot be obtained. Family } \\
\text { intellectual and emotional struggles are eased by } \\
\text { knowing the right time to follow advance } \\
\text { directives/living wills and implications of } \\
\text { following directives. } \\
\text { 3. Planning for discharge from the hospital } \\
\text { 4. Patient/Family values and preferences: valued } \\
\text { individualized discussions that aided in their } \\
\text { understanding of treatments, procedures, etc; } \\
\text { respectful discussions that honored patient and } \\
\text { family wishes and desires }\end{array}$ \\
\hline $\begin{array}{l}\text { Reinke et al. } \\
(2008)\end{array}$ & $\begin{array}{l}\text { Patients with } \\
\text { COPD, } \\
\text { andvanced } \\
\text { cancer, }\end{array}$ & EOL & $\begin{array}{l}\text { Explore transitions regarding palliative } \\
\text { care and end-of-life care from the } \\
\text { perspectives of patients with COPD or }\end{array}$ & $\begin{array}{l}\text { Physicians, patients, and families perceived transitions in } \\
\text { illness differently. Physicians were more likely to identify } \\
\text { hospitalizations and disease related medical changes as } \\
\text { significant transitions in serious illness, while patients and }\end{array}$ \\
\hline
\end{tabular}




\begin{tabular}{|c|c|c|c|c|}
\hline & $\begin{array}{l}\text { family } \\
\text { members, } \\
\text { and formal } \\
\text { care } \\
\text { providers }\end{array}$ & & $\begin{array}{l}\text { advanced cancer, their family members, } \\
\text { physicians, nurses. }\end{array}$ & $\begin{array}{l}\text { families were more likely to identify changes to activities of } \\
\text { daily living or functional changes as significant transitions } \\
\text { in serious illness. } \\
\text { Certain therapies, such as oxygen therapy, were seen as a } \\
\text { positive treatment option by physicians, whereas patients } \\
\text { viewed the initiation of oxygen as another functional } \\
\text { limitation. } \\
\text { Both patients, families and physicians regarded palliative } \\
\text { care as a distinct shift from curative to comfort measures. } \\
\text { There were some differences in perceptions of transitions } \\
\text { among patients with different diseases: patients with cancer } \\
\text { recognized times of improvement in illness as significant, } \\
\text { while patients with COPD did not experience times of } \\
\text { improvement. }\end{array}$ \\
\hline $\begin{array}{l}\text { Russ \& Kaufman } \\
\text { (year) }\end{array}$ & Family & EOL & $\begin{array}{l}\text { Explore family experiences of } \\
\text { communication with physicians and } \\
\text { understandings of prognosis }\end{array}$ & $\begin{array}{l}\text { Desired information to facilitate discussions about care, } \\
\text { however, did not want hope taken away. } \\
\text { Frustrated with vague estimates of time left. Did not } \\
\text { consider death a possibility if not verbalized by physician. } \\
\text { Frustrated with medical jargon } \\
\text { Families felt left out of care conversations } \\
\text { Families expected doctors to take charge of the situation and } \\
\text { drive discussions about care. } \\
\text { Rotating physicians and specialist made it difficult to } \\
\text { coordinate care - lack of communication among physicians } \\
\text { left family with overwhelming need to piece together care } \\
\text { and information } \\
\text { Families felt regret over care decisions that felt "rushed" - } \\
\text { unprepared for decisions required to make. } \\
\text { Discussions at end-of-life limited to consent to withdrawal } \\
\text { treatment. } \\
\text { Families desire guidance throughout illness; need help } \\
\text { interpreting information. }\end{array}$ \\
\hline $\begin{array}{l}\text { Tallman et al., } \\
2012\end{array}$ & $\begin{array}{l}\text { Palliative } \\
\text { care eligible } \\
\text { patients and } \\
\text { families }\end{array}$ & EOL & $\begin{array}{l}\text { Identify the primary needs of patients, } \\
\text { families, and caregivers across the } \\
\text { continuum of care from their vantage } \\
\text { point. } \\
\text { Learn how inpatient palliative care teams } \\
\text { affect the care experience. }\end{array}$ & $\begin{array}{l}\text { Patient, families and caregivers identified multiple needs. } \\
\text { Needs evolved over time. } \\
\text { 1. Sensitive, effective communication about advanced } \\
\text { illness in language that they could understand, with } \\
\text { caring and compassion. } \\
\text { Timely access to coordinated medical care; pain, } \\
\text { discharge care needs often not met }\end{array}$ \\
\hline
\end{tabular}




\begin{tabular}{|c|c|c|c|c|}
\hline & & & & $\begin{array}{l}\text { 2. Respect for and honoring care decisions; where } \\
\text { they would receive care, pressure to make } \\
\text { decisions before ready } \\
\text { 3. Psychological, social and spiritual support; } \\
\text { respectful of individual and cultural differences; } \\
\text { promote sense of meaning and purpose. } \\
\text { 4. Caregiver support; ill equipped to care for patient, } \\
\text { appreciative of hospice care, loss of work time, loss } \\
\text { of freedom }\end{array}$ \\
\hline $\begin{array}{l}\text { Waldrop et al. } \\
\text { (2005) }\end{array}$ & Caregivers & EOL/Hospice & $\begin{array}{l}\text { To understand how caregivers make the } \\
\text { transition to end stage caregiving. }\end{array}$ & $\begin{array}{l}\text { Comprehension of terminality was impacted by information } \\
\text { given by HCP, friends and family, as well as changes in the } \\
\text { ability of the patient to carry out previous activities and } \\
\text { roles. } \\
\text { Families experienced conflicting role responsibilities, } \\
\text { financial burden and role changes as they cared for their ill } \\
\text { family member. } \\
\text { Families reported relying on religious and/or faith support } \\
\text { systems during transition for physical and emotional } \\
\text { support. }\end{array}$ \\
\hline $\begin{array}{l}\text { Wittenberg- } \\
\text { Lyles, } \\
\text { Goldsmith, } \\
\text { Ragan }(2011)\end{array}$ & $\begin{array}{l}\text { Patient and } \\
\text { Family }\end{array}$ & EOL/Hospice & $\begin{array}{l}\text { Determine which communication } \\
\text { characteristics influence early palliative } \\
\text { care and how these shape patient's and } \\
\text { family's experience }\end{array}$ & $\begin{array}{l}\text { Three illness journeys identified: } \\
\text { Isolated - where no palliative care or hospice service was } \\
\text { introduced. This was related to a curative only approach to } \\
\text { care and linear, disease focused conversations about cure. } \\
\text { Rescued - where there was an abrupt shift from cure to } \\
\text { comfort care. This was related to linear, disease focused } \\
\text { conversations about care and prognostic and physiologic } \\
\text { discussions about dying. } \\
\text { Comforted - where palliative and hospice services were } \\
\text { included along the disease continuum. This was related to } \\
\text { conversations about advanced planning, collaboration } \\
\text { among HCP, family involvement, shared decision making, } \\
\text { and holistic care. } \\
\text { Acknowledgement of family's needs and participation t/o } \\
\text { illness facilitated positive transitions. Families valued } \\
\text { inclusion in care and maintaining care preferences. }\end{array}$ \\
\hline $\begin{array}{l}\text { Wright et al., } \\
\text { (2013) }\end{array}$ & $\begin{array}{l}\text { Terminally ill } \\
\text { cancer } \\
\text { patients and } \\
\text { unpaid, }\end{array}$ & Terminal* & $\begin{array}{l}\text { To examine associations between } \\
\text { caregivers' US acculturation levels and } \\
\text { advanced cancer patient's communication } \\
\text { with physicians, treatment preferences, }\end{array}$ & $\begin{array}{l}\text { USAS scores were predictive of patient's preferences for } \\
\text { prognostic information, feeding tubes, and desire to avoid } \\
\text { dying in an ICU. Scores indicating higher caregiver } \\
\text { acculturation were associated with increased odds of }\end{array}$ \\
\hline
\end{tabular}




\begin{tabular}{|l|l|l|l|}
\hline & $\begin{array}{l}\text { informal } \\
\text { caregivers }\end{array}$ & $\begin{array}{l}\text { advance care planning, and cancer } \\
\text { treatment received. }\end{array}$ & $\begin{array}{l}\text { patient participation in clinical trials and greater odds of } \\
\text { patients receiving chemotherapy. } \\
\text { No associations between caregiver's acculturation and } \\
\text { measures of patient's trust in physicians, comfort asking } \\
\text { questions about their care, or communication processes after } \\
\text { adjusting for other confounding variables were detected. } \\
\text { Advanced cancer patients desire for prognostic information, } \\
\text { EOL care preferences, and medical care varied by } \\
\text { caregiver's acculturation scale. }\end{array}$ \\
\hline
\end{tabular}

*Note that in studies, EOL, terminal, palliative care, and hospice were used interchangeably. These terms all referred to seriously ill persons in the last phases of illness, once curative measures had ceased. 


\section{Appendix 3.1}

\section{Introduction}

Again, thank you for participating in my study. As we discussed over the phone, I am very interested in better understanding more about how your family made the transition from the time when you and your family first learned of (name's) diagnosis up through the point when you realized and that medical care would not cure their disease.

I am particularly interested in learning more about how you and your family made sense out of what was happening to (name of person) over time and what you thought you needed to do and who you turned to for help and more information in order to help your (XXXX) Other things that I am interested in learning more about is about what influence friends, community, and/or religious beliefs had in your family's understanding of how families "should" behave during this period of time. Does that make sense or do you have any questions for me before we begin?

\section{Question Guide}

Baseline knowledge systems:

1. Let's start by talking about when you and your family first learned about (name's) diagnosis of XXXX. Tell me what you and you're your family knew or thought about illness and what might happen (knowledge systems)?

2. From what you have said, $X Y Z$ seemed to play a role in how you and your family understood what having XYZ would entail and what might eventually happen. Is there anything else you want to add to that?

Now, let's talk about the period of time after (name) was diagnosed up until you and your family understood that medical treatment could not cure the disease and that it would ultimately lead to their death.

Family/Kinship

1. How did your family react during this period of time?

2. How were decisions made about who would be doing what?

3. What about the roles you and others in your family played in caregiving? In what ways did these change over time?

Knowledge systems

1. Who, if anyone, did you turn to for advice or information about the illness and what might happen as it progressed? Tell me more. 


\section{Community/Social Systems}

1. Talk to me a little bit about your circles of friends or co-workers typically would respond to you and your family. Were there general norms about how families should respond in these situations? Tell me more.

2. Let's talk about the health professionals involved in (name) care. Tell me a little about their communication with you and your family. Tell me more.

\section{Faith/Religion}

1. Talk to me a little bit about how your faith or religious beliefs factored in during this period of time. Tell me more.

So, this is what I have heard up to this point (summarize statements). Would you say that was correct? Is there anything else that you would like to share with me?

\section{Conclusion}

Thank you for participating in this study. I hope that from your's and others' experiences we can learn how to better help families throughout the illness of their loved ones. 


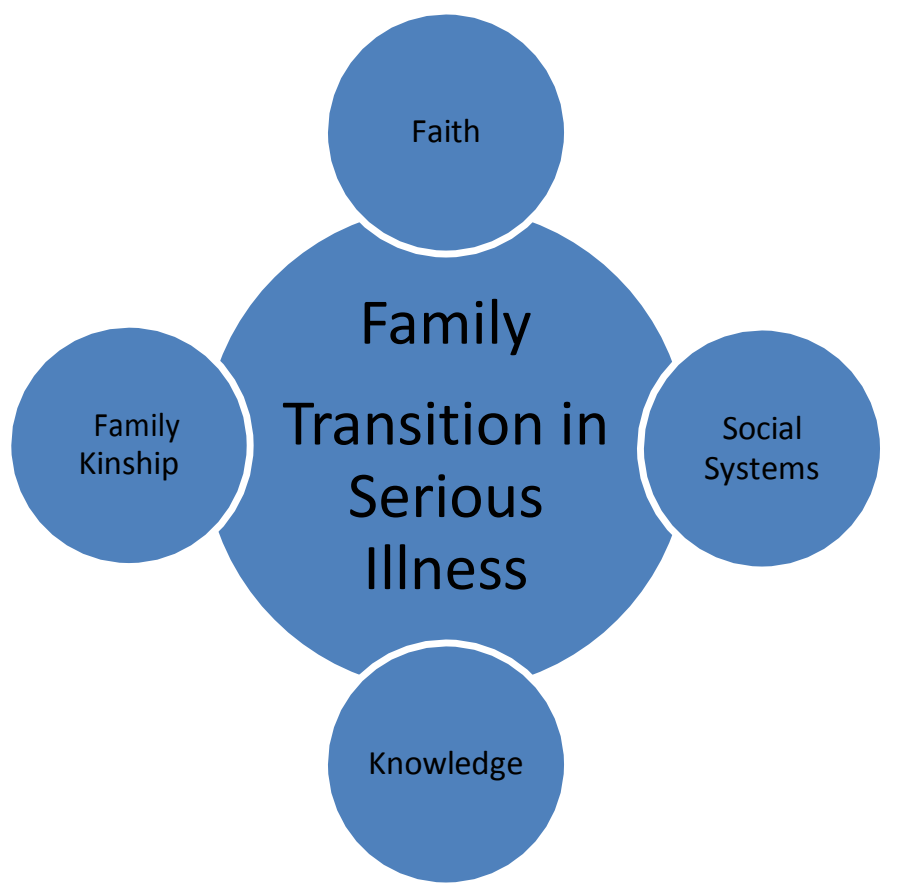


Appendix 3.2

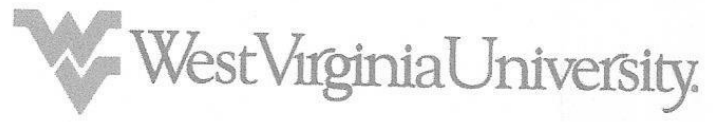

Dear Participant,

This letter is a request for you to take part in a research project to better understand the transitions that families go through during the illness of a family member, and how they come to terms with the illness. This research is being conducted by Veronica Gallo, RN, MSN, a doctoral student in the School of Nursing at WVU under the supervision of Dr. Joy Buck, $\mathrm{RN}, \mathrm{PhD}$, a professor in the School of Nursing. The research is required for a Doctoral Degree in Nursing Research.

Agreeing to participate in this study means that you are willing to participate in a one to two hour interview session with the investigator in a quiet, private location of your choosing. If you agree, the interviews will be digitally recorded and the recordings will be transcribed. All identifiable information provided during the interview will be removed from the transcript. When the transcribed interviews are verified by the researcher, the recordings will be erased. If you are agreeable, the researcher may contact you for a follow-up interview to clarify information from the original interview or to ask additional questions. Because the interviews may cause you to become saddened, the researcher will provide you with a list of counseling services that you may access if you feel it is necessary.

Your involvement in this project will be kept as confidential as legally possible. You will not be asked any information that should lead back to your identity as a participant. No identifiable information disclosed during the interview will be included in the transcripts. All data will be reported in the aggregate so individuals cannot be idenified. You must be 18 years of age or older to participate. Your participation is completely voluntary. You may stop the interview at any time and/or cease your participation in this study at any time. West Virginia University's Institutional Review Board acknowledgement of this project is on file.

I hope that you will participate in this research project, as it could be beneficial in understanding how families make sense of transitions throughout the illness of their family member and how nurses, doctors and other care providers can help families. Thank you very much for your time. Should you have any questions about this letter or the research project, please feel free to contact Veronica Gallo at (304) 476-2930 or by email atvgallo@hsc.wvu.edu.

Thank you for your time and help with this project.

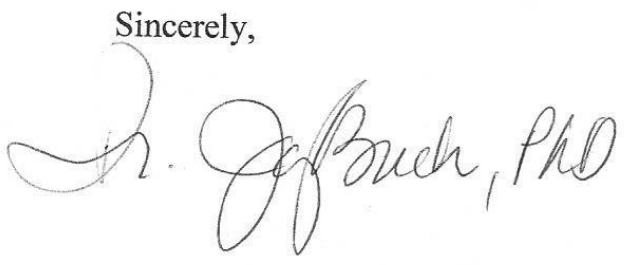




\section{Appendix 4.1}

Table 4.1: Demographic information on study participants and deceased family members

\begin{tabular}{|c|c|c|c|c|c|c|}
\hline Participant Age & Gender & $\begin{array}{l}\text { Participant Relationship } \\
\text { to deceased }\end{array}$ & $\begin{array}{l}\text { Age of } \\
\text { Deceased }\end{array}$ & $\begin{array}{l}\text { Gender } \\
\text { Deceased }\end{array}$ & Illness/Diagnosis & $\begin{array}{l}\text { Time since } \\
\text { death }\end{array}$ \\
\hline 41 & Female & Daughter & 68 & Male & Esophageal Cancer & 3 Months \\
\hline $37 * *$ & Female & Niece/daughter & 68 & Male & Prostate Cancer & 27 months \\
\hline $53^{* *}$ & Male & son & 86 & Female & Parkinson's & 26 months \\
\hline $47^{*}$ & female & Daughter-in-law & 86 & Female & Parkinson's & 26 months \\
\hline 53 & Female & Sister & 63 & Female & Ovarian Cancer & 24 months \\
\hline $53^{*}$ & Male & Son & 74 & Male & Pancreatic Cancer & 3 months \\
\hline $70^{*}$ & Male & Close friend & 71 & male & Pancreatic cancer & 3 months \\
\hline 58 & female & sister & 53 & female & Breast cancer & 12 months \\
\hline $28^{*}$ & female & niece & 54 & male & Cancer of unknown origin & 13 months \\
\hline $73^{* *}$ & female & daughter & 94 & female & $\begin{array}{l}\text { Aortic stenosis, renal } \\
\text { failure }\end{array}$ & 35 months \\
\hline 38 & female & granddaughter & 87 & female & $\mathrm{CHF}$ & 20 months \\
\hline $73 * *$ & male & husband & 70 & female & Pancreatic cancer & 30 months \\
\hline
\end{tabular}

*denotes health care professional

** denotes main caregiver 